\title{
EXCIMER LASER TREATMENTS OF IRON, ALUMINUM AND SILICON SUBSTRATES IN NITROGEN AND METHANE ATMOSPHERES
}

\author{
Dissertation \\ zur Erlangung der Doktorgrades \\ der Mathematisch-Naturwissenschaftlichen Fakultäten \\ der Georg-August-Universität zu Göttingen
}

vorgelegt von

Ettore Carpene

aus Feltre - Italien

Göttingen 2002 
D 7

Referent: $\quad$ PD Dr. Peter Schaaf

Korreferent: $\quad$ Prof. Dr. Wolfgang Felsch

Tag der mündlichen Prüfung: 17.10.2002 
"God created the light, men made it coherent".

EC 


\section{Contents}

\begin{tabular}{ll}
\hline List of Figures & 7 \\
\hline
\end{tabular}

\begin{tabular}{ll}
\hline List of Tables & 10
\end{tabular}

\begin{tabular}{lll}
\hline & Introduction & 11
\end{tabular}

2 Materials properties: structures and phases $\quad 13$

2.1 The iron-carbon and the iron-nitrogen systems . . . . . . . . . . . . . . . . 13

2.2 The aluminum-carbon and the aluminum-nitrogen systems . . . . . . . . . 15

2.3 The silicon-carbon and the silicon-nitrogen systems . . . . . . . . . . . 17

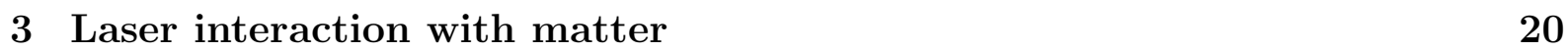

3.1 The heat equation . . . . . . . . . . . . . . . . . . . 20

3.2 Computation of the temperature profiles . . . . . . . . . . . . . . . . . . . 22

3.3 Phenomena occurring on the target surface . . . . . . . . . . . . . . . . . 26

3.3.1 Vaporization . . . . . . . . . . . . . . . . . . 26

3.3 .2 Recondensation . . . . . . . . . . . . . . . . 27

3.3 .3 Plasma formation . . . . . . . . . . . . . . . . . . . . . . . . . . . . 29

\begin{tabular}{|lll}
\hline 4 & Experimental methods & 31
\end{tabular}

4.1 Samples preparation and laser treatments . . . . . . . . . . . . . . . . . . 31

4.2 Rutherford Backscattering Spectrometry . . . . . . . . . . . . . . . . . . . 33

4.3 Resonant Nuclear Reaction Analysis . . . . . . . . . . . . . . . . . . . . . 36

4.4 Mössbauer Spectroscopy . . . . . . . . . . . . . . . . . . . . . . . . . . . 40

4.5 X-Ray Absorption Fine Structure . . . . . . . . . . . . . . . . . . . . . . . 44

4.6 Crystallography and X-Ray Diffraction . . . . . . . . . . . . . . . . . 47

4.7 Nanoindentation hardness . . . . . . . . . . . . . . . . . . . . 52 


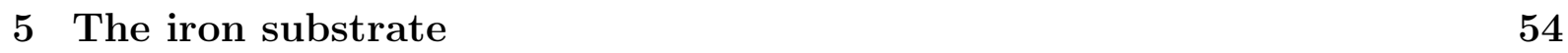

5.1 The nitrogen transport mechanism . . . . . . . . . . . . . . . . 54

5.1 .1 The diffusion process $\ldots \ldots \ldots \ldots$. . . . . . . . . . 54

5.1 .2 The melting depth . . . . . . . . . . . . . . . 57

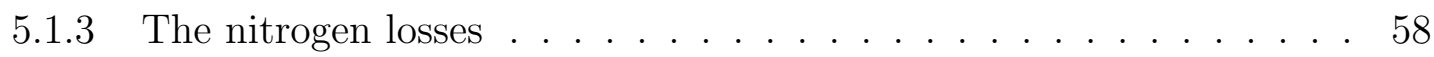

5.1 .4 The results . . . . . . . . . . . . . . . . . . . . . . . 58

5.2 Laser irradiation in methane atmosphere . . . . . . . . . . . . . . . . . . . 61

5.2 .1 Influence of the meandering spot overlap . . . . . . . . . . . . . 61

5.2 .2 Influence of the methane gas pressure . . . . . . . . . . . . . . . 65

$5.2 .3 \quad$ Formation of a homogeneous cementite layer . . . . . . . . . . . . . 67

\begin{tabular}{|lll}
\hline 6 & The aluminum substrate & 71
\end{tabular}

6.1 Laser nitriding of aluminum . . . . . . . . . . . . . . . . . . 71

$6.1 .1 \quad$ Preliminary investigations: the laser fluence dependence . . . . . . 71

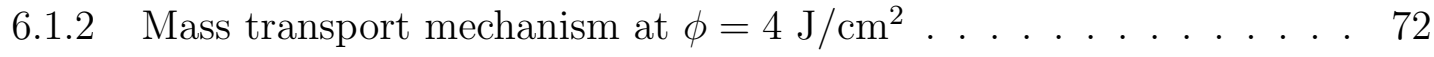

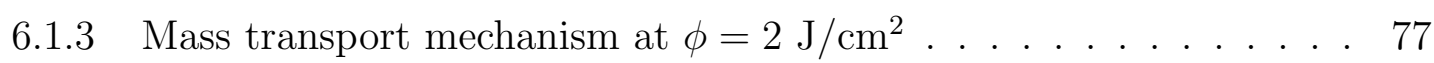

6.1 .4 Crystal structures and mechanical properties . . . . . . . . . . . . 81

6.2 Laser irradiation in methane atmosphere . . . . . . . . . . . . . . . . 84

\begin{tabular}{lll}
\hline 7 & The silicon substrate & 87
\end{tabular}

$7.1 \quad$ Laser irradiation in methane atmosphere . . . . . . . . . . . . . . . . . . . 88

$7.1 .1 \quad$ Influence of the number of pulses . . . . . . . . . . . . . . . 88

7.1 .2 Influence of the methane pressure . . . . . . . . . . . . . . . . . . 91

7.2 Laser irradiation in nitrogen atmosphere . . . . . . . . . . . . . . . . . . . 93

$7.2 .1 \quad$ Influence of the number of pulses . . . . . . . . . . . . . . . . 93

7.2 .2 The segregation problem . . . . . . . . . . . . . . 97

$\begin{array}{lll}8 & \text { Conclusions and outlooks } & 101\end{array}$

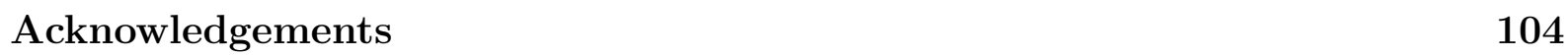

\begin{tabular}{lr}
\hline Bibliography & 106
\end{tabular} 


\section{List of Figures}

2.1 The iron-carbon phase diagram . . . . . . . . . . . . . . . . . . . . 14

2.2 The iron-nitrogen phase diagram . . . . . . . . . . . . . . . . . . . . . . 14

2.3 The aluminum-carbon phase diagram . . . . . . . . . . . . . . . . . . . . . 16

2.4 The aluminum-nitrogen phase diagram . . . . . . . . . . . . . . . . . . . . 16

2.5 The silicon-carbon phase diagram . . . . . . . . . . . . . . . . . . . 17

2.6 The silicon-nitrogen phase diagram . . . . . . . . . . . . . . . . . . . . . 18

$3.1 \quad$ Temperature dependence of $c_{p}(\mathrm{~T})$ and $\kappa(\mathrm{T})$ for Fe, $\mathrm{Al}$ and $\mathrm{Si}$. . . . . . . . 24

3.2 Surface temperature profiles and melting depths of Fe and Al vs. time. . . 25

3.3 Si surface temperature with T-dependent and T-independent $R_{0}$. . . . . 25

3.4 Sketch of the irradiation geometry: the Knudsen layer . . . . . . . . . . . . 27

3.5 Vapor pressures and densities within and outside the Knudsen layer . . . . 28

$3.6 \quad$ Influence of the Si surface temperature on the vapor pressure . . . . . . . . 28

4.1 Sketch of the laser irradiation experimental setup . . . . . . . . . . . . . . 32

4.2 Sketch of the single spot and the meandering treatments . . . . . . . . . . 33

$4.3 \quad$ Sketch of the RBS experiment . . . . . . . . . . . . . . . . . . . . . . 33

$4.4 \quad$ RBS spectrum of a thin Fe film $(90 \mathrm{~nm})$ deposited on a Si substrate . . . . 35

4.5 Sketch of the RNRA experiment . . . . . . . . . . . . . . . . . 36

$4.6 \quad$ Stopping cross sections of a proton beam in pure Fe, $\mathrm{Al}$ and $\mathrm{N}$ (gas). . . . 38

4.7 Conversion of the $Y_{Q}(E)$ to $f(z) \ldots \ldots$. . . . . . . . . . . . . . . . . . . 39

4.8 Depth resolution of a $430 \mathrm{keV} \mathrm{H}^{+}$beam traversing $\mathrm{Al}$ and $\mathrm{Fe}$. . . . . . . . 40

$4.9 \quad{ }^{57} \mathrm{Fe}$ Mössbauer transitions scheme and $\alpha$-Fe Mössbauer spectrum . . . . . 42

4.10 Geometries of the TMS, the CEMS and the CXMS experiments . . . . . . 43

$4.11 \mu, \mu_{0}$ and $\chi$ of a Si sample . . . . . . . . . . . . . . . 45 
4.12 Sketch of the L.U.R.E. SA32 beamline experimental setup . . . . . . . . 46

4.13 Bragg and von Laue explanations of the X-Ray Diffraction . . . . . . . . 47

4.14 The $\mathrm{fcc}$ Bravais lattice $\ldots \ldots \ldots$

4.15 Angular dependence of $\phi_{L P} e^{-2 M}$ for Fe and Al substrates . . . . . . . 50

4.16 Geometry of the GIXRD experiment $\ldots \ldots \ldots \ldots \ldots$

4.17 Indentation depth vs. load of a single crystalline Si substrate . . . . . . . . 52

4.18 Corrected and non-corrected hardness of a single crystalline Si substrate . 53

$5.1 \quad$ Isotopic experiments $1^{*} / 0,1^{*} / 1,1^{*} / 2,1^{*} / 4 \ldots \ldots \ldots \ldots$

5.2 Diffusion length squared of the depth profiles vs. the number of pulses . . 56

5.3 Evolution of $C_{n}$ and $\left(D t_{e}\right)_{n}$ vs. the number of pulses $\ldots \ldots \ldots$

5.4 The nitrogen profile after 8 laser pulses $\ldots \ldots \ldots \ldots$

$5.5 \quad$ Nitrogen take-up and nitrogen losses per pulse $\ldots \ldots \ldots$. . . . . . . . 60

$5.6 \quad$ Measured and simulated nitrogen depth profiles (the pulse series) $\ldots . . .60$

5.7 CEM spectra of the meander scans $8 \times 8,11 \times 12$ and $16 \times 16$ in $\mathrm{CH}_{4} \ldots 62$

5.8 Atomic arrangements of the $\epsilon-\mathrm{Fe}_{3} \mathrm{C}$ carbide $\ldots \ldots \ldots \ldots$

5.9 Phase fraction vs. spot overlap: CEMS and GIXRD analyses . . . . . . . . 63

5.10 Hardness curves of the meandered samples . . . . . . . . . . . . . . 64

5.11 CEM spectra of $11 \times 12$ scan at various methane pressures $\ldots \ldots . .65$

5.12 CEM phase fractions vs. methane gas pressure $\ldots \ldots \ldots \ldots 6$

5.13 Average carbon content vs. methane gas (CEMS and RBS) . . . . . . . 67

5.14 RBS spectrum of the sample treated in $\mathrm{CH}_{4}$ with the $16 \times 16 \mathrm{scan} \ldots . .68$

5.15 CEM and CXM spectra of the sample irradiated in $\mathrm{CH}_{4} \ldots \ldots 68$

5.16 GIXRD diffractograms $\left(5^{\circ}, 10^{\circ}, 15^{\circ}\right)$ of the cementite layer $\ldots . . .69$

5.17 Temperature-carbon activity phase diagram of the Fe-C system $\ldots . .70$

$6.1 \quad$ Fluence dependence of the aluminum nitriding efficiency $\ldots \ldots \ldots 72$

6.2 Simulation of the Al maximum surface temperature vs. the laser fluence . 73

$6.3 \quad$ Nitrogen depth profiles of the pulse series at $4 \mathrm{~J} / \mathrm{cm}^{2}$ in 1 bar $\mathrm{N}_{2} \ldots 73$

6.4 Nitrogen depth profiles of the pulse series at $4 \mathrm{~J} / \mathrm{cm}^{2}$ in 6 bar $\mathrm{N}_{2} \ldots 74$

6.5 Surface temperature profile of pure $\mathrm{Al}$ irradiated at $4 \mathrm{~J} / \mathrm{cm}^{2} \ldots \ldots . .74$

6.6 Average nitrogen content vs. the number of pulses in 1 and 6 bar $\mathrm{N}_{2} \ldots 76$ 
6.7 Pressure dependence of the $S_{\text {in }}$ parameter . . . . . . . . . . . . . . . 76

6.8 Nitrogen depth profiles of the pulse series at $2 \mathrm{~J} / \mathrm{cm}^{2}$ in 1 bar $\mathrm{N}_{2} \ldots$. . . 77

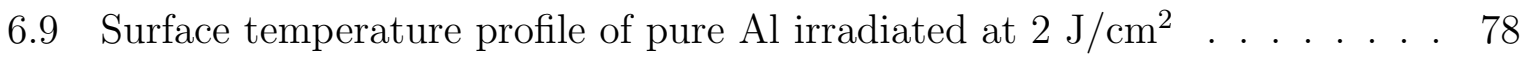

6.10 TEM picture of sample irradiated 256 times at $2 \mathrm{~J} / \mathrm{cm}^{2}$ in 1 bar $\mathrm{N}_{2} \ldots$. . 78

6.11 Temperature gradient of the Al sample irradiated at $2 \mathrm{~J} / \mathrm{cm}^{2} \ldots \ldots$. . . . 80

6.12 Isotopic experiments $256^{*} / 0,256^{*} / 4,256^{*} / 8,256^{*} / 16$ at $2 \mathrm{~J} / \mathrm{cm}^{2}$. . . . 80

6.13 Schematic view of the mass transport mechanism at $2 \mathrm{~J} / \mathrm{cm}^{2}$. . . . . . . . 82

6.14 GIXRD of the samples irradiated at $2 \mathrm{~J} / \mathrm{cm}^{2}$ and $4 \mathrm{~J} / \mathrm{cm}^{2}$ in $1 \mathrm{bar}_{2}$. . . 83

6.15 Hardness measurements vs. the number of pulses and the $\mathrm{N}_{2}$ pressure . . . 84

6.16 Correlation between the surface hardness and the average nitrogen content 84

6.17 RBS carbon depth profile of the aluminum sample meandered in $\mathrm{CH}_{4}$. . . 85

6.18 Hardness profile of the laser carburized Al substrate . . . . . . . . . . . . . 85

6.19 GIXRD patter of the laser carburized Al substrate . . . . . . . . . . . . . 86

7.1 $\quad$ EXAFS oscillations of Si, $\beta$-SiC, $\alpha$-Si ${ }_{3} \mathrm{~N}_{4}$, Si irradiated in $\mathrm{CH}_{4}$ and in $\mathrm{N}_{2}$. 87

$7.2 \quad$ FT of the virgin Si, the Si sample irradiated in $\mathrm{CH}_{4}$ and the $\beta$-SiC . . . . . 88

7.3 BFT: Si irradiated in $\mathrm{CH}_{4}$ with 256 laser shots $\ldots . . . . . . .89$

$7.4 \quad \mathrm{SiC}$ phase fraction vs. number of laser pulses . . . . . . . . . . . . . . . . . 90

7.5 GIXRD: Si sample irradiated in $\mathrm{CH}_{4}$ with 256 pulses . . . . . . . . . . . . 91

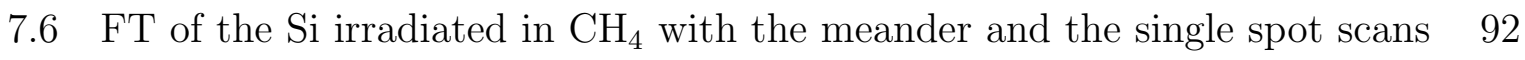

$7.7 \quad$ FT of the samples meandered in $0.5,1$ and 4 bar $\mathrm{CH}_{4}$. . . . . . . . . . . . 92

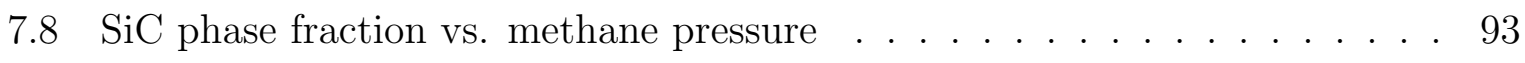

$7.9 \quad$ FT of the virgin Si, the Si sample irradiated in $\mathrm{N}_{2}$ and the $\alpha-\mathrm{Si}_{3} \mathrm{~N}_{4} \quad \ldots \quad$. . 94

7.10 BFT: Si irradiated in $\mathrm{N}_{2}$ with 256 laser shots . . . . . . . . . . . . . . . . . 95

$7.11 \mathrm{Si}_{3} \mathrm{~N}_{4}$ phase fraction vs. number of laser pulses . . . . . . . . . . . . . . . 95

7.12 FT of the Si irradiated in $\mathrm{N}_{2}$ with the meander and the single spot scans . 96

7.13 Nitrogen depth profiles of the single spot treatments . . . . . . . . . . . . 98

7.14 The melting depths vs. time of Si irradiated at $4 \mathrm{~J} / \mathrm{cm}^{2}$ and $\mathrm{Al}$ at $2 \mathrm{~J} / \mathrm{cm}^{2} \quad 98$

7.15 Mass transport during the raw-beam meandering . . . . . . . . . . . . . 99

7.16 Nitrogen depth profiles of Si meandered at various laser fluences . . . . . . 100 


\section{List of Tables}

2.1 Crystallographic properties of the Fe-C and the Fe-N systems . . . . . . . 15

2.2 Crystallographic properties of the Al-C and the Al-N systems . . . . . . . 17

2.3 Crystallographic properties of the Si-C and the Si-N systems . . . . . . . 18

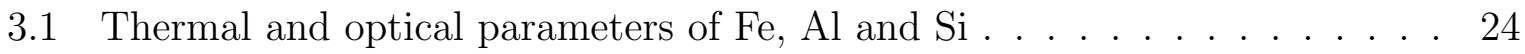

4.1 Basic features of the Siemens XP 2020 excimer laser . . . . . . . . . . . . 31

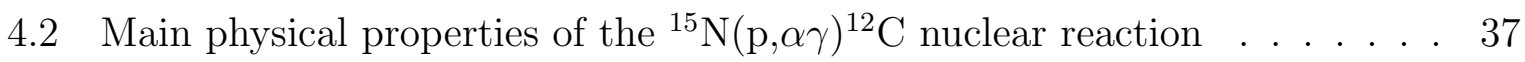

$4.3 \quad$ Angular dependence of the Mössbauer transition lines . . . . . . . . . . . . 43

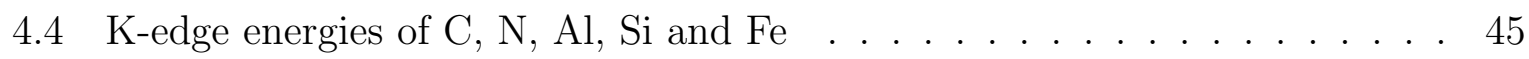

4.5 Crystal systems and Bravais lattices . . . . . . . . . . . . . . . . . . . . . . 49

4.6 GIXRD information depth vs. incidence angle . . . . . . . . . . . . . . . . 51

$5.1 \quad$ Parameters used for the nitrogen transport model in $\mathrm{Fe}$. . . . . . . . . . . 61

$5.2 \quad$ Fits of the hardness profiles vs. the spot overlap $\left(\mathrm{Fe}\right.$ in $\left.\mathrm{CH}_{4}\right)$. . . . . . . . 64

$5.3 \quad$ CEMS and CXMS parameters of the cementite layer . . . . . . . . . . . . 69

6.1 List of the main thermal and physical parameters of Al and AlN . . . . . . 81

6.2 Results of the Rietveld refinement of $\mathrm{Al} / \mathrm{AlN}$. . . . . . . . . . . . . . . . . 83

7.1 Three-shells fitting of the Si samples irradiated in $\mathrm{CH}_{4} \quad$. . . . . . . . . . . 90

7.2 Two-shells fitting of the Si samples irradiated in $\mathrm{N}_{2}$. . . . . . . . . . . . . 96 


\section{Chapter 1}

\section{Introduction}

Material processing with laser beams is an expanding field with fascinating physics and attractive technological applications. An increasing number of laser-based treatments have been developed in the last decades: laser cutting [1] and welding [2, laser alloying [3], laser hardening [4] and cladding are few of the numerous examples. Large interest is also addressed to Laser-induced Chemical Vapor Deposition (LCVD, see Ref. [5], ch. 16) and Pulse Laser Deposition (PLD, see Ref. [5], ch. 22) as successful techniques to synthesize polymer films, high temperature superconductors, diamond-like carbon (DLC) and, more generally, metastable compounds. Besides their applications in laser drilling [6] and patterning [7], ultrafast (fs) lasers are nowadays becoming extremely promising for the production of collimated $\mathrm{MeV}$ ion beams [8]. Among all branches of laser processing, the irradiation of metals and semiconductors in reactive atmospheres is a relatively new technique. When the reactive gas contains nitrogen, the process is called laser nitriding. If a carbon-containing atmosphere is used, the laser treatment is termed laser carburizing. Nitriding and carburizing are common methods to improve the mechanical and tribological properties of metals [9]. The incorporation of nitrogen and carbon can be achieved by means of several standard methodologies, such as gas nitriding and carburizing [10, 11, 12, 13, 14], plasma processing [15, 16, 17, 18, 19, 20] and ion implantation [21, 22, 23, 24, 25, 26, 27], just to mention few of them. In the present work, iron, aluminum and silicon substrates have been treated in controlled nitrogen and methane atmospheres with a pulsed excimer laser $\left(\lambda=308 \mathrm{~nm}, t_{p}=55 \mathrm{~ns}\right)$, aiming to initiate the reaction between the irradiated target and the atmospheric environment, thus incorporating nitrogen and carbon into the surface layers. The choice of the substrates has been motivated by the many technological applications of the pure materials as well as their nitrides/carbides counterparts. Iron laser nitriding has been the subject of many investigations in the last decade, and it is now well established how the laser can efficiently incorporate a considerable amount of nitrogen (>30 at.\%), leading to enhanced mechanical properties of the surface (see [28, 29, 30, 31] and references therein). Similarly to nitrogen, it will be shown here that also carbon can be successfully incorporated in the iron matrix, if the laser treatment is performed in methane atmosphere. Both aluminum nitride $(\mathrm{AlN})$ and silicon carbide $(\mathrm{SiC})$ have many outstanding properties and numerous structural, physical and thermal similarities, making them perfect candidates for the laser synthesis in reactive atmospheres, as it will be shown in the up-coming chapters. Among the advantages of the laser treatment in reactive atmospheres, the rapidity, 
the simple experimental setup and the ability to modify only the surface layer (up to a few microns), leaving the bulk unchanged, should be mentioned. It is therefore an excellent tool for the synthesis of coatings, with the advantage that the modified surface layer is partially originating from the substrate itself, thus enhancing its adhesion to the underlying material. The technological importance of coatings is testified by the large number of conferences and journals dedicated to their characterization [32] and to the synthesis of protective [33, 34, tribological [35] and optoelectronics [36, 37] coatings. From a more general viewpoint, the present work could be allocated in the framework of the photon-induced surface processing that includes all surface treatments and modifications achieved by photon beams. In the following Chapter 2 a brief review on the main properties of the $\mathrm{X}-\mathrm{N}$ and the $\mathrm{X}-\mathrm{C}$ systems $(\mathrm{X}=\mathrm{Fe}, \mathrm{Al}, \mathrm{Si})$ will be given, with special attention to the phase diagrams. Chapter 3 will describe the basic physics governing the laser interaction with matter, focusing on the heat equation (section 3.1), the computational procedure (section 3.2) and the phenomena occurring on the surface of the irradiated targets (section 3.3). In Chapter 4 the experimental methods used to investigate the processed samples will be introduced: the specimens preparation and treatment (section 4.1), the Rutherford Backscattering Spectrometry (section 4.2), the Resonant Nuclear Reaction Analysis (section 4.3), the Mössbauer Spectroscopy (section 4.4), the X-ray Absorption Fine Structure (section 4.5), the X-ray Diffraction (section 4.6) and the nanoindentation method (section 4.7). Chapters 5, 6 and 7 will report the experimental results of the laser irradiation respectively of iron, aluminum and silicon substrates in nitrogen and methane gases. In particular, sections 5.1 and 6.1 will focus on the nitrogen mass transport mechanisms in iron and in aluminum, while sections 5.2 and 6.2 will discuss the laser treatment of iron and aluminum in methane. In sections 7.1 and 7.2 the analysis of the silicon substrates irradiated in methane and in nitrogen atmospheres, respectively, will be reported. The conclusions of the work and the outlooks for future developments and investigations will be drawn in Chapter 8 . 


\section{Chapter 2}

\section{Materials properties: structures and phases}

The subject of the present work is the laser irradiation of iron, aluminum and silicon substrates in nitrogen and methane atmospheres. As it will be shown, during the laser treatment, nitrogen and carbon are incorporated into the target, leading to the formation of nitrides and carbides. The basic feature of the binary systems $\mathrm{X}-\mathrm{N}$ and $\mathrm{X}-\mathrm{C}(\mathrm{X}=\mathrm{Fe}$, $\mathrm{Al}, \mathrm{Si}$ ) will be described in the following sections with special emphasis on the features of the phase diagrams. The thermal and physical properties will be reported in Chapter 3 , where the laser-material interaction is illustrated.

\subsection{The iron-carbon and the iron-nitrogen systems}

The Fe-C and the Fe-N binary systems have been extensively studied in the last century, due to their technological importance, especially in metallurgy. Their equilibrium phase diagrams are reported in Fig. 2.1 and Fig. 2.2. Both systems are characterized by the presence of solid solutions as well as stoichiometric compounds. The Fe-C system can be described in terms of the stable graphite or in terms of the metastable $\mathrm{Fe}_{3} \mathrm{C}$ (dashed line in Fig. 2.1). The maximum solubility of carbon in $\alpha$-Fe (bcc) is $\sim 0.1$ at. $\%$ at $740^{\circ} \mathrm{C}$, while it reaches the value of $\sim 9$ at. $\%$ in $\gamma$-Fe (fcc) at $1153^{\circ} \mathrm{C}$. The only iron-carbide predicted by the phase diagram is $\theta-\mathrm{Fe}_{3} \mathrm{C}$ (also known as cementite) that crystallizes in the orthorhombic structure Pnma. Under equilibrium conditions a carbon content higher than 25 at.\% leads to the precipitation of graphite in the iron matrix. Other metastable $\mathrm{Fe}-\mathrm{C}$ phases have been reported in the literature such as $\mathrm{Fe}_{2} \mathrm{C}$ (in hexagonal and orthorhombic forms), $\chi-\mathrm{Fe}_{5} \mathrm{C}_{2}$ (also known as $\mathrm{Hägg}$ carbide), $\mathrm{Fe}_{7} \mathrm{C}_{3}, \mathrm{Fe}_{20} \mathrm{C}_{9}$ and $\mathrm{Fe}_{23} \mathrm{C}_{6}$ [38, 39]. The hexagonal $\epsilon-\mathrm{Fe}_{x} \mathrm{C}$ can exist in a wide compositional range, typically with $2<x<6$ [39]. Most of them are considered transition carbides, i.e. during the tempering process of carbon steel the sequence of carbides formation can be described by the following scheme [39]:

$$
\text { carbon clusters } \rightarrow \text { ordered } \epsilon \text { phase } \rightarrow \chi \rightarrow \theta,
$$

with cementite being the final product. $\theta-\mathrm{Fe}_{3} \mathrm{C}$ has great technological importance for the mechanical properties of steels and iron alloys, however it is hard to obtain as a 


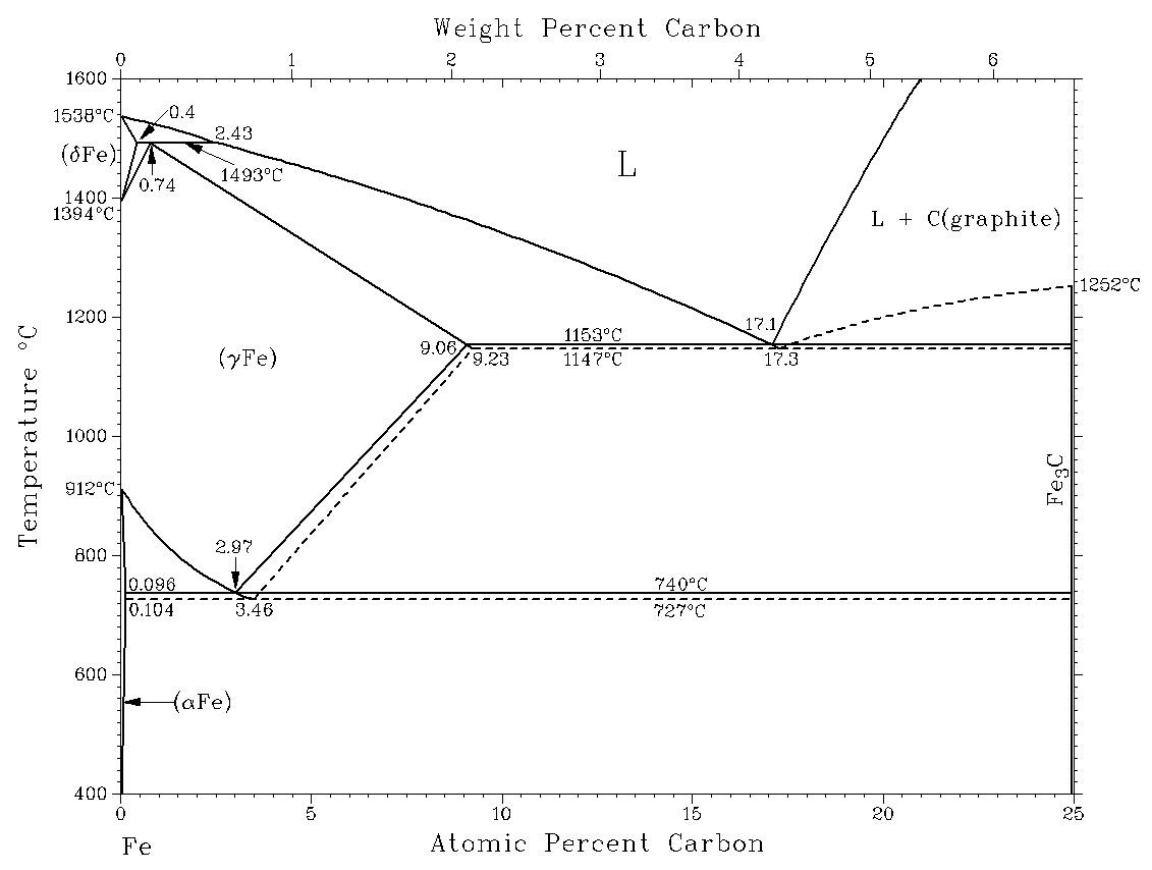

Figure 2.1: The iron-carbon phase diagram [38].

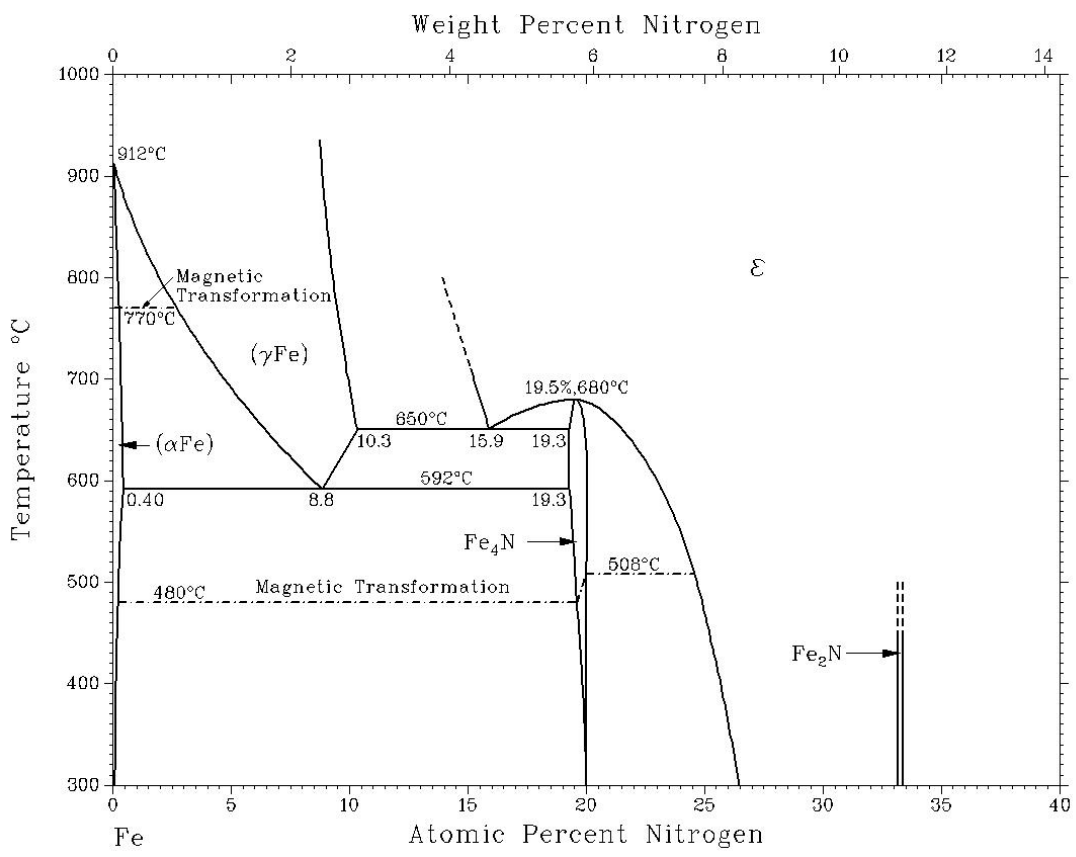

Figure 2.2: The iron-nitrogen phase diagram [38].

single phase, since it is typically embedded in the steel matrix. Due to this difficulty, only few investigations of the $\theta-\mathrm{Fe}_{3} \mathrm{C}$ electronic structure [40], its mechanical [41] and thermodynamical [42] properties can be found in the literature. Recently, a single-phase cementite film has been prepared by a special Physical Vapor Deposition technique [41, and no other method has yet been successful in synthesizing pure cementite. A thorough review on the iron-carbon system can be found in Ref. [39, 42]. Some crystallographic 
Table 2.1: Crystallographic properties of the Fe-C and the Fe-N systems.

\begin{tabular}{|c|c|c|c|c|c|c|c|}
\hline \multirow[t]{2}{*}{ Phase } & \multirow[t]{2}{*}{ Space group } & \multicolumn{3}{|c|}{ Lattice constants $[\AA]$} & \multirow[t]{2}{*}{$\rho\left[\mathrm{g} / \mathrm{cm}^{3}\right]$} & \multirow[t]{2}{*}{$\mathrm{T}_{m}\left[{ }^{\circ} \mathrm{C}\right]$} & \multirow[t]{2}{*}{$\mathrm{T}_{b}\left[{ }^{\circ} \mathrm{C}\right]$} \\
\hline & & $\mathrm{a}$ & $\mathrm{b}$ & $\mathrm{c}$ & & & \\
\hline$\alpha$-Fe & $\operatorname{Im} 3 \mathrm{~m}$ & 2.866 & & & 7.86 & $912^{(a)}$ & \\
\hline$\gamma-\mathrm{Fe}$ & Fm3m & 3.572 & & & 8.14 & $1394^{(b)}$ & \\
\hline$\delta$-Fe & & $-(\mathrm{sam}$ & as $\alpha-\mathrm{l}$ & e) & - & 1538 & 2750 \\
\hline$\alpha^{\prime}-\mathrm{FeC}_{0.042}$ & $\mathrm{I} 4 / \mathrm{mmm}$ & 2.856 & & 2.960 & 7.47 & & \\
\hline$\epsilon-\mathrm{Fe}_{2} \mathrm{C}$ & $\mathrm{P} 6_{3} / \mathrm{mmc}$ & 2.754 & & 4.349 & 7.19 & & \\
\hline$\theta-\mathrm{Fe}_{3} \mathrm{C}$ & Pnma & 5.091 & 6.743 & 4.526 & 7.68 & 1252 & \\
\hline$\chi-\mathrm{Fe}_{5} \mathrm{C}_{2}$ & $\mathrm{C} 2 / \mathrm{c}$ & 11.560 & 4.573 & 5.058 & 7.60 & & \\
\hline $\mathrm{Fe}_{7} \mathrm{C}_{3}$ & $\mathrm{P} 6_{3} / \mathrm{mc}$ & 6.882 & & 4.540 & 7.62 & & \\
\hline$\gamma^{\prime}-\mathrm{Fe}_{4} \mathrm{~N}$ & $\mathrm{P} \overline{4} 3 \mathrm{~m}$ & 3.795 & & & 7.21 & $680^{(c)}$ & \\
\hline$\alpha "-\mathrm{Fe}_{16} \mathrm{~N}_{2}$ & $\mathrm{I} 4 / \mathrm{mmm}$ & 5.720 & & 6.290 & 7.45 & & \\
\hline$\epsilon-\mathrm{Fe}_{3} \mathrm{~N}$ & $\mathrm{P}_{3} 22$ & 2.695 & & 4.362 & 7.36 & & \\
\hline$\zeta-\mathrm{Fe}_{2} \mathrm{~N}$ & Pbnm & 5.523 & 4.830 & 4.425 & 7.07 & $200^{(d)}$ & \\
\hline
\end{tabular}

(a) Transforms into $\gamma$ phase.

(b) Transforms into $\delta$ phase.

(c) Transforms into $\epsilon$ phase.

(d) Dissolves.

and thermal properties are summarized in Table 2.1 (data from [38, 43, 44]). Similarly to carbon, nitrogen atoms occupy interstitial sites in the iron lattice. There are 5 equilibrium solid phases in the Fe- $\mathrm{N}$ system [45]: $\alpha-\mathrm{Fe}(\mathrm{N}), \gamma-\mathrm{Fe}(\mathrm{N}), \gamma^{\prime}-\mathrm{Fe}_{4} \mathrm{~N}, \zeta-\mathrm{Fe}_{2} \mathrm{~N}$ and the $\epsilon$ phase. In the $\alpha$-Fe, the maximum nitrogen solubility is $\sim 0.4$ at. $\%$ at $592^{\circ} \mathrm{C}$, but it can reach $\sim 10.3$ at. $\%$ at $650^{\circ} \mathrm{C}$ in the $\gamma$-Fe. The stoichiometric $\gamma^{\prime}-\mathrm{Fe}_{4} \mathrm{~N}$ phase (also known as roaldite) exists in a narrow region that extends up to $680^{\circ} \mathrm{C}$, crystallizing in the cubic $\mathrm{P} \overline{4} 3 \mathrm{~m}$ structure. The hcp $\epsilon-\mathrm{Fe}_{x} \mathrm{~N}$ phase is stable at elevated pressures and crystallizes with structure similar to the $\epsilon-\mathrm{Fe}_{x} \mathrm{C}$ carbide in a large compositional range. The existence of the metastable $\alpha$ "- $-\mathrm{Fe}_{16} \mathrm{~N}_{2}$ [39, 42] and the bct martensite phases has been reported as well [39].

\subsection{The aluminum-carbon and the aluminum- nitrogen systems}

Pure aluminum crystallizes in the fcc structure and has a rather low melting point $\left(660^{\circ} \mathrm{C}\right)$, compared to other metals. The incorporation of carbon or nitrogen in the metal leads to a substantial increase of the melting temperature, but the extremely low solubilities of $\mathrm{C}$ and $\mathrm{N}$ in $\mathrm{Al}$ hinder the formation of solid solutions. Instead, stoichiometric compounds are formed. The equilibrium phase diagrams of the Al-C and the Al-N systems are reported in Fig. 2.3 and Fig. 2.4. The only stable aluminum carbide is $\mathrm{Al}_{4} \mathrm{C}_{3}$ with a melting point of $2173^{\circ} \mathrm{C}$ and hexagonal crystallographic structure (space group R $\overline{3} \mathrm{~m}$ ). The solubility of carbon in aluminum is controversial: the maximum value reported in the literature is 


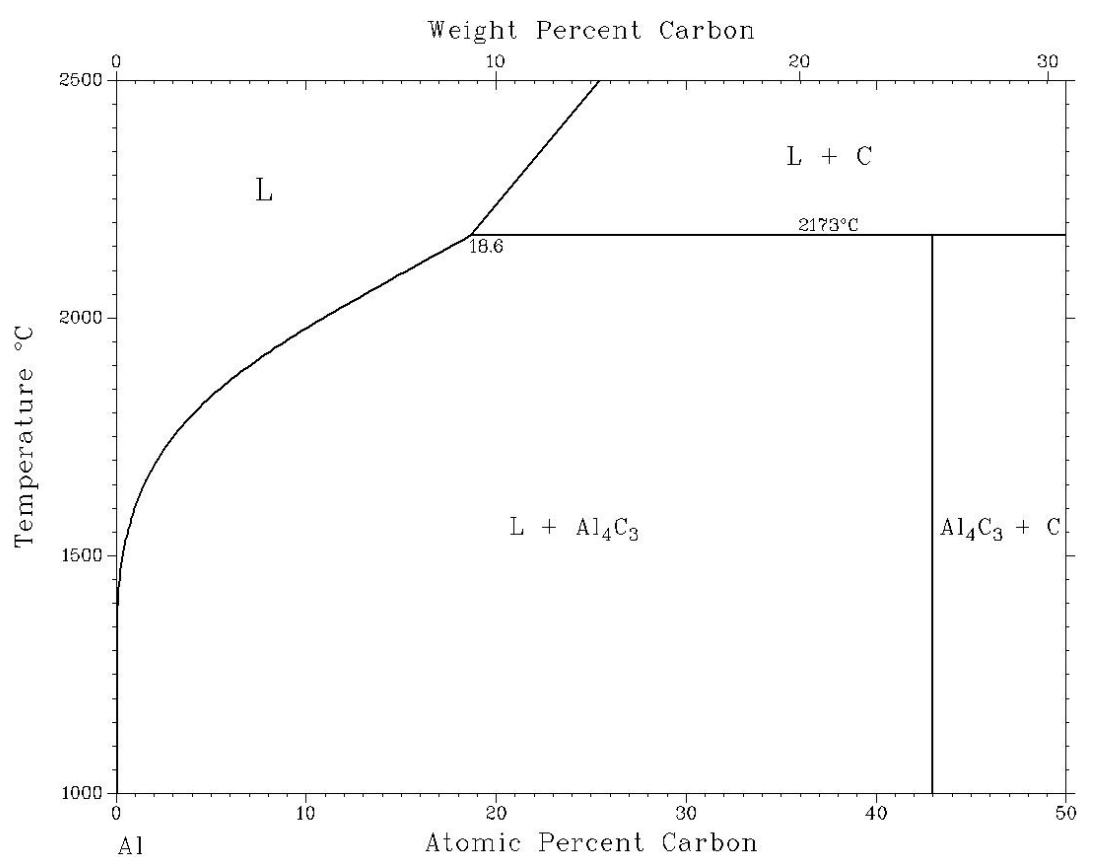

Figure 2.3: The aluminum-carbon phase diagram [38].

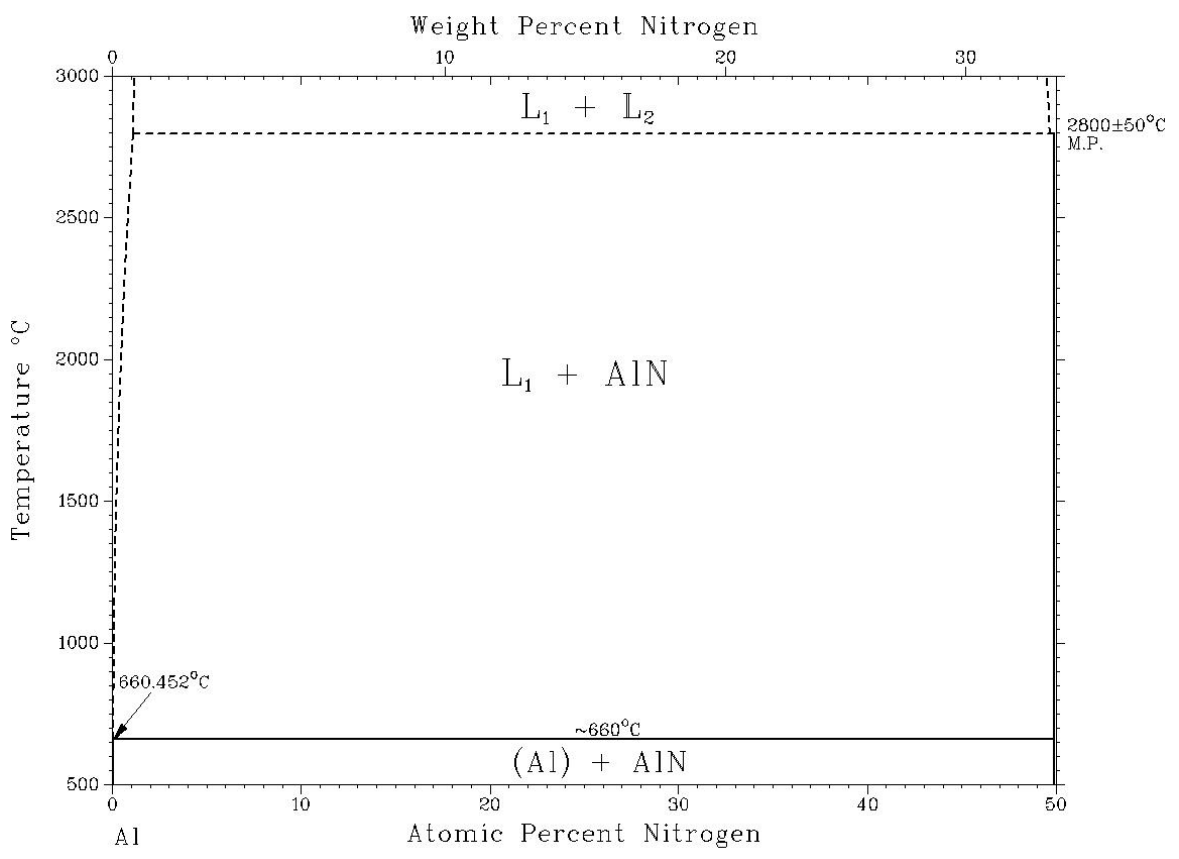

Figure 2.4: The aluminum-nitrogen phase diagram [38].

0.71 at. $\%$ (at $1200^{\circ} \mathrm{C}$ ) [46], but it is believed to be unrealistically high [47]. The Al-N phase diagram is primarily qualitative, due to the lack of data on the composition range of the liquid and the solid phases. According to thermodynamic calculations, the solubility of nitrogen in aluminum at its melting point is about $10^{-11}$ at.\%, but it is believed to increase to few at.\% at the nitride melting point $\left(2800^{\circ} \mathrm{C}\right.$ [38] $)$. AlN is known to dissociate at about $2100^{\circ} \mathrm{C}$ under standard conditions, but the dissociation temperature increases with the ambient pressure [48]. The stable nitride has wurtzite-type structure, transforming into $\mathrm{NaCl}$-type at elevated pressures (several GPa [49]). The basic crystallographic properties 
of pure $\mathrm{Al}, \mathrm{Al}_{4} \mathrm{C}_{3}$ and $\mathrm{AlN}$ are reported in Table 2.2. AlN has several outstanding physical

Table 2.2: Crystallographic properties of the Al-C and the Al-N systems.

\begin{tabular}{|c|c|c|c|c|c|c|}
\hline \multirow[t]{2}{*}{ Phase } & \multirow[t]{2}{*}{ Space group } & \multicolumn{2}{|c|}{ Lattice constants $[\AA]$} & \multirow[t]{2}{*}{$\overline{\rho \rho}\left[\mathrm{g} / \mathrm{cm}^{3}\right]$} & \multirow[t]{2}{*}{$\mathrm{T}_{m}\left[{ }^{\circ} \mathrm{C}\right]$} & \multirow[t]{2}{*}{$\overline{\mathrm{T}_{b}\left[{ }^{\circ} \mathrm{C}\right]}$} \\
\hline & & $\mathrm{a}$ & $\mathrm{c}$ & & & \\
\hline $\mathrm{Al}$ & Fm31 & 4.049 & & 2.70 & 660 & 2457 \\
\hline $\mathrm{Al}_{4} \mathrm{C}_{3}$ & $\mathrm{R} \overline{3} \mathrm{~m}$ & 3.338 & 24.99 & 2.97 & 2173 & \\
\hline AlN (wurtzite) & $\mathrm{P}_{6} / \mathrm{mc}$ & 3.111 & 4.979 & 3.26 & $2100^{(a)}$ & \\
\hline AlN (NaCl-type) & Fm3m & 4.045 & & 4.11 & $2100^{(a)}$ & \\
\hline
\end{tabular}

${ }^{(a)}$ Dissolves.

properties, that makes it a very attractive material. It has high hardness (10.8 GPa [50]) and resistance to chemicals, high thermal conductivity $\kappa(2 \mathrm{~W} / \mathrm{cm} \mathrm{K}$ at room temperature [43]) and wide band gap $(6.2 \mathrm{eV}$ [51]). Besides, the ability to form continuous alloys with GaN and InN opens a wide range of possibilities for tunable optical devices [51, 52].

\subsection{The silicon-carbon and the silicon-nitrogen sys- tems}

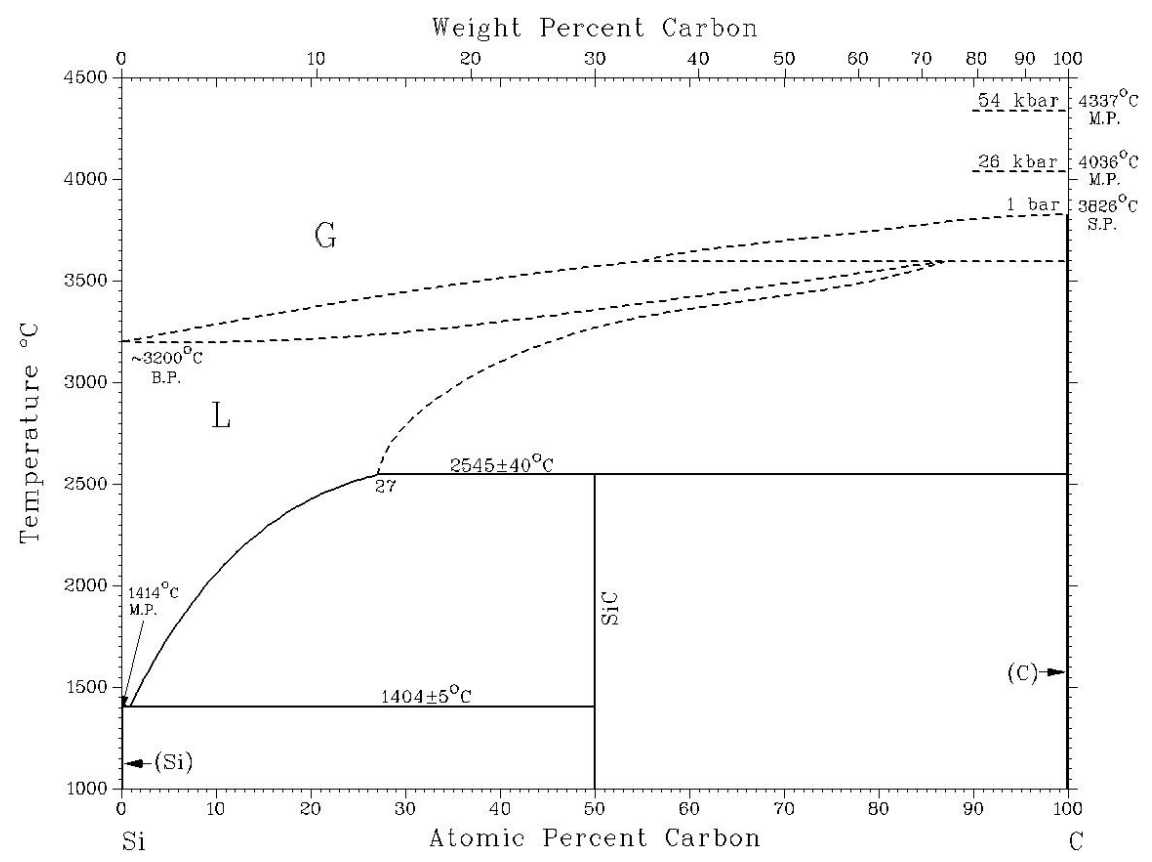

Figure 2.5: The silicon-carbon phase diagram [38].

Silicon is no doubt the most investigated material of the last century. All electronic devices of nowadays life are based on the silicon technology. It is a semiconductor (band gap of $1.12 \mathrm{eV}[53]$ ) that crystallizes in the characteristic diamond-type cubic cell. The equilibrium phase diagrams of the $\mathrm{Si}-\mathrm{C}$ and the $\mathrm{Si}-\mathrm{N}$ systems are reported in Fig. 2.5 and 
Fig. 2.6. The solid solubilities of carbon and nitrogen in silicon are quite low $\left(\sim 10^{-3}\right.$ at.\%

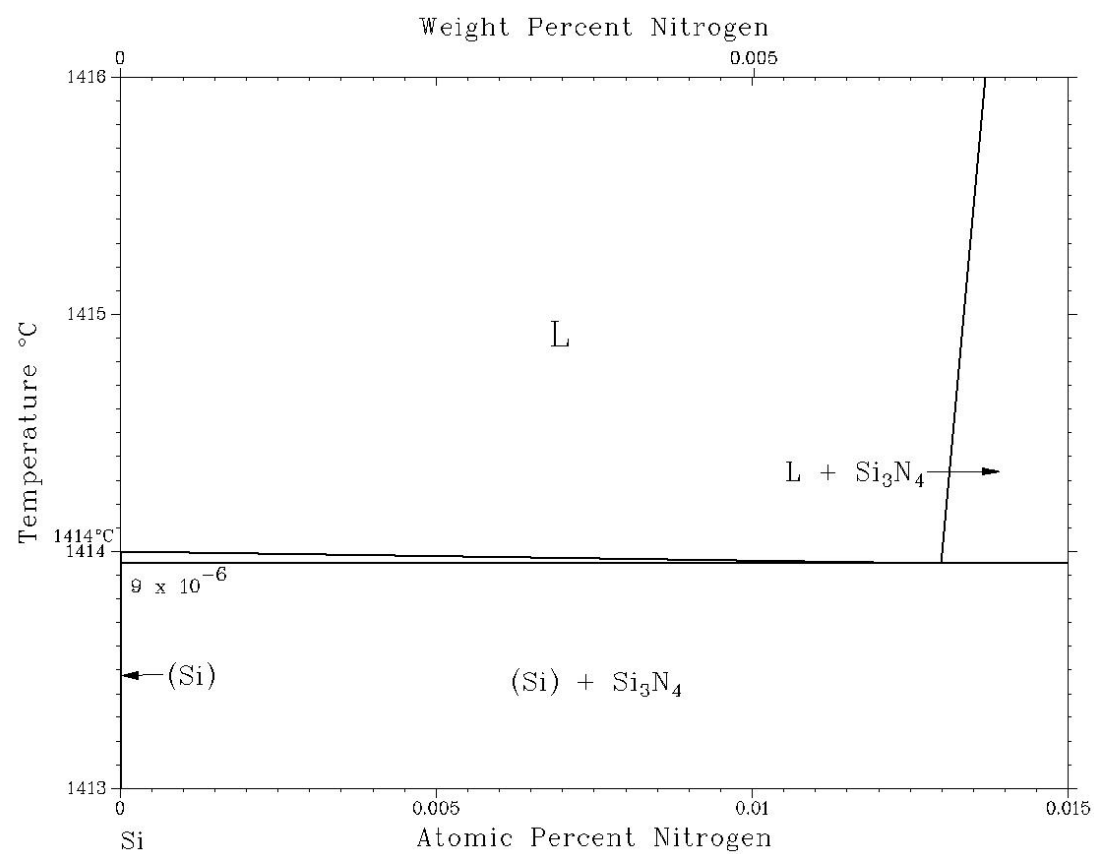

Figure 2.6: The silicon-nitrogen phase diagram [38.

of $\mathrm{C}$ at $1300^{\circ} \mathrm{C}$ and 0.02 at.\% of $\mathrm{N}$ in liquid Si [38]). Carbon dissolves substitutionally in

Table 2.3: Crystallographic properties of the Si-C and the Si-N systems.

\begin{tabular}{|c|c|c|c|c|c|c|}
\hline \multirow[t]{2}{*}{ Phase } & \multirow[t]{2}{*}{ Space group } & \multicolumn{2}{|c|}{ Lattice constants $[\AA]$} & \multirow[t]{2}{*}{$\rho\left[\mathrm{g} / \mathrm{cm}^{3}\right]$} & \multirow[t]{2}{*}{$\overline{\mathrm{T}} \mathrm{T}_{m}\left[{ }^{\circ} \mathrm{C}\right]$} & \multirow[t]{2}{*}{$\overline{\mathrm{T}_{b}\left[{ }^{\circ} \mathrm{C}\right]}$} \\
\hline & & $\mathrm{a}$ & c & & & \\
\hline $\mathrm{Si}$ & Fd3m & 5.430 & & 2.33 & 1410 & 2355 \\
\hline$\alpha-\mathrm{SiC}(2 \mathrm{H})$ & $\mathrm{P} 6_{3} / \mathrm{mc}$ & 3.081 & 5.031 & 3.22 & $2830^{(a)}$ & \\
\hline$\beta-\mathrm{SiC}(3 \mathrm{R})$ & $\mathrm{F} \overline{4} 3 \mathrm{~m}$ & 4.358 & & 3.22 & 2900 & \\
\hline$\alpha-\mathrm{Si}_{3} \mathrm{~N}_{4}$ & P31c & 7.754 & 5.621 & 3.18 & $1900^{(b)}$ & \\
\hline$\beta-\mathrm{Si}_{3} \mathrm{~N}_{4}$ & $\mathrm{P} 6_{3} \mathrm{~m}$ & 7.604 & 2.907 & 3.20 & $1900^{(b)}$ & \\
\hline $\mathrm{c}-\mathrm{Si}_{3} \mathrm{~N}_{4}$ & $\mathrm{Fd} \overline{3} \mathrm{~m}$ & 7.760 & & 3.93 & $430^{(b)}$ & \\
\hline
\end{tabular}

(a) Sublimates.

(b) Dissolves.

silicon, forming the stoichiometric $\mathrm{SiC}$ carbide with the hexagonal $(\alpha-\mathrm{SiC})$ or the cubic $(\beta$-SiC $)$ structure, the former being less stable at any temperature below the peritectic point $\left(2545^{\circ} \mathrm{C}, 27\right.$ at.\% of $\mathrm{C}$ [38]). The hexagonal carbide is generally termed $2 \mathrm{H}, 4 \mathrm{H}$ or $6 \mathrm{H}$ according to the dimension of the lattice parameter $\mathrm{c}(\sim 5, \sim 10$ or $\sim 15 \AA$, respectively). The only nitride phase of the $\mathrm{Si}-\mathrm{N}$ system is $\mathrm{Si}_{3} \mathrm{~N}_{4}$, existing in three polymorphs: $\alpha-\mathrm{Si}_{3} \mathrm{~N}_{4}, \beta-\mathrm{Si}_{3} \mathrm{~N}_{4}$ (both hexagonal [38, 44]) and the cubic spinel c- $\mathrm{Si}_{3} \mathrm{~N}_{4}$ [54]. The $\alpha$ phase is oxygen-stabilized, although the oxygen content necessary to have a stable compound is controversial [38]. The cubic phase was synthesized recently by laser heating of $\mathrm{Si}_{3} \mathrm{~N}_{4}$ powder in a diamond cell under rather extreme conditions (2000 K, 15 GPa). Its crystallographic space group has been identified by selected-area electron diffraction as $\mathrm{Fd} \overline{3} \mathrm{~m}$ 
with a lattice parameter of $7.76 \AA$ [54]. Both $\mathrm{SiC}$ and $\mathrm{Si}_{3} \mathrm{~N}_{4}$ (in all their polymorphs) are materials with many technological applications. The hexagonal $\mathrm{SiC}$, in particular, is a wide band gap semiconductor $(2.9 \mathrm{eV}[52])$ with an excellent chemical resistance and a high thermal conductivity $(5 \mathrm{~W} / \mathrm{cm} \mathrm{K} \mathrm{[43])} \mathrm{that} \mathrm{makes} \mathrm{it} \mathrm{attractive} \mathrm{for} \mathrm{high-temperature}$ semiconductor applications. The crystallographic properties of $\mathrm{SiC}$ and $\mathrm{Si}_{3} \mathrm{~N}_{4}$ are reported in Table 2.3. 


\section{Chapter 3}

\section{Laser interaction with matter}

In the following chapter the main feature of the laser interaction with matter will be illustrated. The discussion will focus on the effects of the laser beam on the irradiated target, and the description will be carried out in terms of the one-dimensional heat equation 1 In order to produce any effect on the material, the laser light must be absorbed. A laser-induced process is thermally activated if the thermalization of the excitation energy is fast compared to the initial processing step (i.e. the pulse duration) [5]. In such a case, the laser treatment is thermal and the laser can be simply considered a heat source. In metals, light is absorbed by the electrons in the conduction band: the time between electron-electron collisions is of the order of $10^{-14} \mathrm{~s}$, while the electron-phonon relaxation time is $10^{-12}$ to $10^{-10} \mathrm{~s}[53]$. In semiconductors like silicon, similar relaxation times are found for the electron-phonon interaction. In non-metals, interband excitations of electrons can last $10^{-12}$ to $10^{-6} \mathrm{~s}[5$. Therefore, for a laser pulse duration of a few tens of nanoseconds $\left(\sim 10^{-8} \mathrm{~s}\right)$, the laser-induced process in metals and semiconductors can be considered a purely thermal process.

\subsection{The heat equation}

As the laser energy is absorbed, the irradiated sample can undergo structural modifications (incorporation and diffusion of dopants, phase changes, melting, vaporization, etc.) that are correlated to the local temperature. The temperature distribution produced by the absorbed laser radiation can be calculated using the heat equation. In general, the temperature $T=T(\mathbf{r}, t)$ is function of the spatial coordinates $\mathbf{r}$ and the time $t$. In the absence of heat transport by convection or thermal radiation the heat equation has the following general form:

$$
\frac{\rho c_{p}(T)}{M} \frac{\partial T(\mathbf{r}, t)}{\partial t}=-\nabla J_{\text {heat }},
$$

where $\rho\left[\mathrm{g} / \mathrm{cm}^{3}\right]$ is the mass density of the target, $M[\mathrm{~g} /$ mole $]$ is its molar mass, $c_{p}(T)[\mathrm{J}$ $\mathrm{K}^{-1}$ mole $\left.^{-1}\right]$ is its molar specific heat, and $J_{\text {heat }}$ includes all the sources of heat flux. For

\footnotetext{
${ }^{1}$ Terminology note: fast pulsed laser beam is refereed to a pulse duration $t_{p}$ (full width at half maximum) typically in the nanosecond range. The laser beam of energy $E$ is optically focused on an area $A$ of the target, and the irradiance $I_{0}$ is defined as the power per unit area, i.e. $I_{0}=E / A t_{p}$, while the quantity $\phi=E / A$ is called fluence, although it is often termed energy density with an abuse of terminology.
} 
the materials investigated in this work and considering that the overall duration of the laser-induced thermal effects is $\sim 1 \mu$ s (see Fig. 3.2), it can be shown that the thermal diffusion length never exceeds a few $\mu \mathrm{m}$. Since the dimension of the laser spot is several orders of magnitude larger ( $\sim 5 \mathrm{~mm}$ ), the one-dimensional approximation along the beam direction $\widehat{z}$ (normal to the sample surface) can be used, and Eq. (3.1) becomes:

$$
\frac{\rho c_{p}(T)}{M} \frac{\partial T(z, t)}{\partial t}=-\frac{\partial J_{h e a t}}{\partial z}
$$

The heat flux $J_{\text {heat }}$ can be divided into two components: the flux $J_{\text {cond }}$ due to the thermal conduction and the flux $J_{\text {laser }}$ due to the absorbed laser radiation. The latter is simply:

$$
J_{\text {laser }}=I_{a}(z, t),
$$

with $I_{a}$ being the portion of the total laser irradiance $I_{0}\left[\mathrm{~W} / \mathrm{cm}^{2}\right]$ that is not reflected at the sample surface, and therefore $I_{a}=I_{0}\left(1-R_{0}\right)$, where $R_{0}=R_{0}(\lambda, T)$ is the reflectivity of the target for the given laser wavelength $\lambda$ at the temperature $T$. According to Beer's law [5],

$$
\frac{\partial I_{a}(z, t)}{\partial z}=-\alpha I_{a}(z, t)
$$

where $\alpha=\alpha(\lambda, T)$ is the linear absorption coefficient $\left[\mathrm{cm}^{-1}\right]$. The typical value of $\alpha$ in the near UV wavelength range is about $10^{6} \mathrm{~cm}^{-1}$ for silicon and several metals. Eq. (3.4) describes the attenuation of the laser beam inside the irradiated material and for a finite, temperature-independent $\alpha$ we have:

$$
\frac{\partial I_{a}(z, t)}{\partial z}=-\alpha\left(1-R_{0}\right) I_{0}(t) e^{-\alpha z}
$$

In absorbing media, the reflectivity and the absorption coefficient are related to the complex refractive index $\mathbf{n}=n+i k$ by the following relations [3]:

$$
\begin{aligned}
R_{0} & =\frac{(n-1)^{2}+k^{2}}{(n+1)^{2}+k^{2}} \\
\alpha & =\frac{4 \pi k}{\lambda} .
\end{aligned}
$$

The values of $R_{0}$ and $\alpha$, or alternatively $n$ and $k$, are given in the literature for many pure solids and for a number of compounds in a wide range of laser wavelengths [5, 43]. The time dependence of the laser irradiance $I_{0}(t)$ is represented by the following analytical expression that matches the experimental pulse shape quite satisfactorily:

$$
I_{0}(t)=\frac{\phi t}{\omega^{2}} e^{-\frac{t^{2}}{2 \omega^{2}}}
$$

with $\phi$ being the laser fluence $\left[\mathrm{J} \mathrm{cm}^{-2}\right], t_{p}=1.6 \omega$ the pulse duration FWHM and $t \geq 0$. The heat flux due to the thermal conduction is simply:

$$
J_{\text {cond }}=-\kappa(T) \frac{\partial T}{\partial z}
$$

where $\kappa(T)\left[\mathrm{W} \mathrm{cm}^{-1} \mathrm{~K}^{-1}\right]$ is the heat conductivity of the target as a function of the local temperature. When the solid-liquid phase transformation occurs, the molar latent heat 
of melting $L_{m}[\mathrm{~J} / \mathrm{mole}]$ is an heat sink that must be included in the heat equation. A convenient way is to define a quantity that uniquely describes the state of the material as a function of the temperature [3]:

$$
d H(T)= \begin{cases}c_{p} d T & \text { if } T<T_{m} \\ c_{p} d T+L_{m} & \text { if } T \geq T_{m}\end{cases}
$$

where $T_{m}$ is the melting point of the substrate. When the material changes phase, the extra heat $\operatorname{sink} L_{m}$ is "automatically" included in the heat equation. Eqs. (3.3) to (3.10) can be inserted in Eq. (3.2) obtaining the heat equation in the following explicit form:

$$
\frac{\rho}{M} \frac{\partial H}{\partial t}=-\frac{\partial\left(J_{\text {cond }}+J_{\text {laser }}\right)}{\partial z}=\frac{\partial}{\partial z}\left[\kappa(T) \frac{\partial T}{\partial z}\right]+\alpha\left(1-R_{0}\right) \frac{\phi t}{\omega^{2}} e^{-\frac{t^{2}}{2 \omega^{2}}} e^{-\alpha z} .
$$

The surface of the sample is a discontinuity that requires a boundary condition. When the temperature is high enough evaporation can take place, and neglecting the heat losses due to radiation, the average flux $j\left[\right.$ mole $\left.\mathrm{cm}^{-2} \mathrm{~s}^{-1}\right]$ of material removed due to the liquid-vapor phase transition can be computed as follows [3]:

$$
j=p(T) / \sqrt{2 \pi R T M},
$$

with $R$ being the gas constant and $p(T)$ the vapor pressure at the surface temperature $T$. $p(T)$ is obtained from the Clausius-Clapeyron equation [3]:

$$
p(T)=p_{0} \exp \left[\frac{L_{e v}}{R}\left(\frac{1}{T_{b}}-\frac{1}{T}\right)\right] .
$$

where $L_{e v}[\mathrm{~J} / \mathrm{mole}]$ is the molar latent heat of evaporation and $T_{b}$ the boiling point at the reference pressure $p_{0}$. The heat flux due to evaporation is simply:

$$
J_{e v}=j L_{e v},
$$

leading to the following boundary condition at the target surface:

$$
\left.\kappa(T) \frac{\partial T}{\partial z}\right|_{z=0}=J_{e v}=\left.\left(\frac{L_{e v} p_{0}}{\sqrt{2 \pi R T M}} \exp \left[\frac{L_{e v}}{R}\left(\frac{1}{T_{b}}-\frac{1}{T}\right)\right]\right)\right|_{z=0} .
$$

As it will be shown in the following sections, during the laser irradiation an enormous pressure $\left(\sim 10^{2}\right.$ bar $)$ is acting on the target surface. According to Eq. (3.13) the boiling point is shifted to a higher temperature and the liquid can be heated above $T_{b}$, making the transformation described in Eqs. (3.10) unnecessary for the liquid-vapor phase transition. Eq. (3.11) and Eq. 3.15) completely describe the temperature profile in the irradiated sample. Due to the temperature dependence of $\kappa(T)$ and $c_{p}(T)$ the analytical solution is not always available, and the equations must be solved numerically with the finite differences method [55].

\subsection{Computation of the temperature profiles}

With the finite differences method, the time $t$ and space $z$ are divided into discrete intervals $t^{i}=i \Delta t$ and $z_{j}=j \Delta z$ ( $i$ and $j$ are integer numbers). The partial derivation in time is calculated as follows:

$$
c_{p}(T) \frac{\partial T}{\partial t}=c_{p}\left(T_{j}^{i}\right) \frac{T_{j}^{i+1}-T_{j}^{i}}{\Delta t} .
$$


The space derivative of the heat conduction flux $J_{\text {cond }}$ (see Eq. (3.11) ) is:

$$
\frac{\partial}{\partial z}\left[\kappa(T) \frac{\partial T}{\partial z}\right]=\frac{1}{\Delta z}\left[\left(\frac{\kappa\left(T_{j+1}^{i}\right)+\kappa\left(T_{j}^{i}\right)}{2}\right) \frac{T_{j+1}^{i}-T_{j}^{i}}{\Delta z}-\left(\frac{\kappa\left(T_{j}^{i}\right)+\kappa\left(T_{j-1}^{i}\right)}{2}\right) \frac{T_{j}^{i}-T_{j-1}^{i}}{\Delta z}\right] .
$$

In order to conserve the heat flux and to control the discontinuity points of $\kappa$ (typically at any phase transition), the average value of $\kappa$ for two adjacent layers has been used [56]. Since the space is now divided into finite slab, the derivative of the heat flux $J_{\text {laser }}$ due to the absorbed laser energy (see Eq. (3.11)) is calculated as the average irradiance absorbed in one slab divided by the slab thickness:

$$
\frac{\partial I_{a}(z, t)}{\partial z}=\frac{\left.\left\langle I_{a}(z, t)\right\rangle\right|_{\Delta z}}{\Delta z}=\frac{\int_{z}^{z+\Delta z} I_{a}\left(z^{\prime}, t\right) d z^{\prime}}{\Delta z}=-\frac{\left(1-R_{0}\right) I_{0}(t) e^{-\alpha z}\left(1-e^{-\alpha \Delta z}\right)}{\Delta z},
$$

and in terms of finite differences:

$$
\frac{\partial I_{a}(z, t)}{\partial z}=-\frac{\left(1-R_{0}\right)}{\Delta z} \frac{\phi i \Delta t}{\omega^{2}} e^{-\frac{(i \Delta t)^{2}}{2 \omega^{2}}} e^{-\alpha(j-1) \Delta z}\left(1-e^{-\alpha \Delta z}\right)
$$

The boundary condition (Eq. (3.15) ) is included in the surface layer $(j=1)$ :

$$
\left.\kappa(T) \frac{\partial T}{\partial z}\right|_{z=0}=\left(\frac{\kappa\left(T_{2}^{i}\right)+\kappa\left(T_{1}^{i}\right)}{2}\right) \frac{T_{2}^{i}-T_{1}^{i}}{\Delta z}=\frac{L_{e v} p_{0}}{\sqrt{2 \pi R T_{1}^{i} M}} \exp \left[\frac{L_{e v}}{R}\left(\frac{1}{T_{b}}-\frac{1}{T_{1}^{i}}\right)\right] .
$$

Eqs. (3.10) are implemented in the computation as follows: the temperature of a given layer $j$ at a given time $i$ will be $T_{j}^{i}$. During the heating of the sample, the temperature will increase with increasing time: $T_{j}^{i+1} \geq T_{j}^{i}$. When the melting point is reached $T_{j}^{i+1} \geq$ $T_{m} \geq T_{j}^{i}$. If the amount of heat $\Delta H=c_{p}\left(T_{j}^{i}\right)\left(T_{j}^{i+1}-T_{m}\right) \leq L_{m}$, the layer $j$ starts to melt, the new temperature is held to $T_{j}^{i+1}=T_{m}$ and the fraction $\Delta H / L_{m}$ of the slab is molten. On the other hand, if $\Delta H=c_{p}\left(T_{j}^{i}\right)\left(T_{j}^{i+1}-T_{m}\right)>L_{m}$ the whole slab is molten, the extra heat $\Delta H^{\prime}=c_{p}\left(T_{j}^{i}\right)\left(T_{j}^{i+1}-T_{m}\right)-L_{m}$ can be written as $\Delta H^{\prime}=c_{p}\left(T_{j}^{i}\right)\left(\widetilde{T}_{j}^{i+1}-T_{m}\right)$ and the new temperature of the layer is $\widetilde{T}_{j}^{i+1}>T_{m}$. In a similar way, during the solidification we will have $T_{j}^{i+1} \leq T_{m} \leq T_{j}^{i}$ and the procedure is repeated mutatis mutandis, with the proper signs. The melting depth vs. time is easily calculated, since at any time step $i$, all the layers $j$ satisfying the condition $T_{j}^{i} \geq T_{m}$ are in the liquid phase. The heat equation in finite differences becomes an algebraic equation that can be solved with respect to $T_{j}^{i+1}$. Starting with initial condition $T_{j}^{i}=298 \mathrm{~K}$ (room temperature) for every $i$ and $j$, the temperature is calculated iteratively for each layer at each time step. The values of $\Delta z$ and $\Delta t$ are not arbitrarily chosen, but they must satisfy the Neumann criterion [57]:

$$
\Delta t<\Delta z^{2}\left[\frac{\rho c_{p}(T)}{2 \kappa(T)}\right]_{\text {minimum }}
$$

in order to obtain convergent, physical solutions. The whole computational procedure has been implemented in a FORTRAN computer code using typical values of $\Delta z \sim 10$ $\mathrm{nm}$, and $\Delta t \sim 1 \mathrm{ps}$, depending on the thermal properties of the target. Table 3.1 reports the thermal and optical parameters of $\mathrm{Fe}, \mathrm{Al}$ and $\mathrm{Si}$ used in the thermal calculations, while Fig. 3.1 shows the temperature dependence of $\kappa(T)$ and $c_{p}(T)$ for the pure iron, aluminum and silicon substrates. Fig. 3.2 represents the time evolution of the surface 
Table 3.1: Thermal and optical parameters of iron, aluminum and silicon used in the heat equation (data from [43]).

\begin{tabular}{lrrr}
\hline \hline & $\mathrm{Fe}$ & $\mathrm{Al}$ & $\mathrm{Si}$ \\
\hline$M[\mathrm{~g} / \mathrm{mole}]$ & 56 & 27 & 28 \\
$\rho\left[\mathrm{g} / \mathrm{cm}^{3}\right]$ & 7.86 & 2.7 & 2.33 \\
$L_{m}[\mathrm{~kJ} / \mathrm{mole}]$ & 15 & 10.5 & 49.8 \\
$L_{e v}[\mathrm{~kJ} /$ mole $]$ & 350 & 296 & 420 \\
$T_{m}[\mathrm{~K}]$ & 1810 & 933.5 & 1685 \\
$T_{b}[\mathrm{~K}]$ & 3023 & 2740 & 2628 \\
$R_{0}(\lambda=308 \mathrm{~nm})$ & 0.53 & $0.5^{(a)}$ & 0.6 \\
$\alpha\left[\mathrm{cm}^{-1}\right](\lambda=308 \mathrm{~nm})$ & $1 \times 10^{6}$ & $1.5 \times 10^{6}$ & $1.5 \times 10^{6}$ \\
\hline \hline$(a)$ Measured. & & &
\end{tabular}

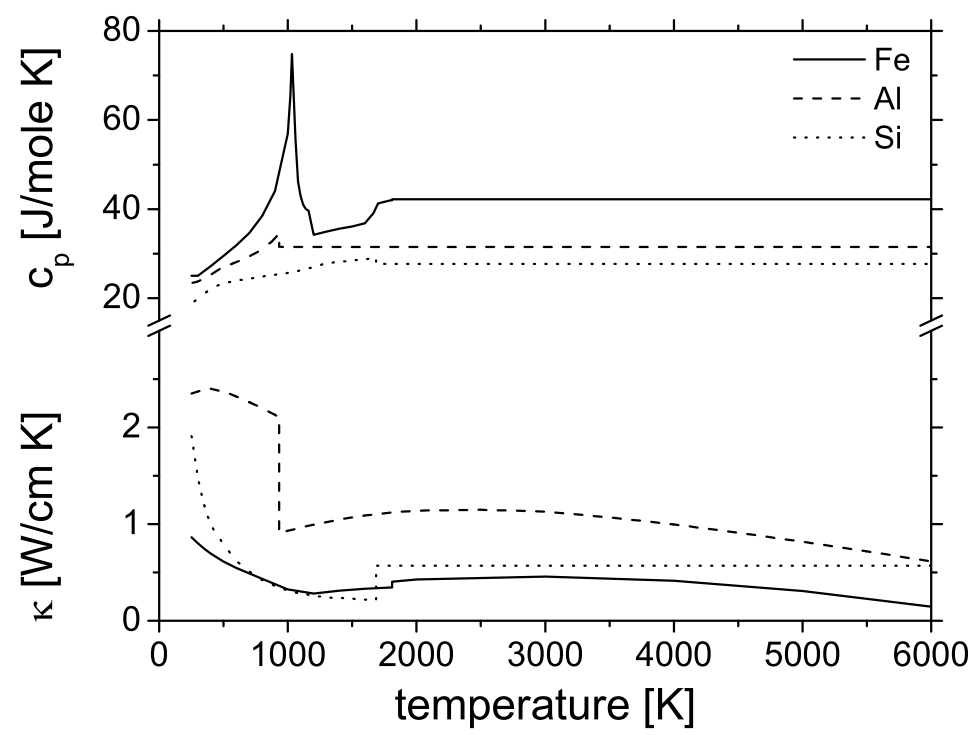

Figure 3.1: Temperature dependence of the molar specific heat (top) and the thermal conductivity (bottom) of iron (solid line), aluminum (dashed line) and silicon (dotted line). Data from [58.

temperature profiles $T_{1}^{i}$ and the melting depths of the iron and the aluminum targets irradiated at a laser fluence $\phi=4 \mathrm{~J} / \mathrm{cm}^{2}$ with a laser pulse duration $t_{p}=55 \mathrm{~ns}(\mathrm{XeCl}$ excimer laser). Although it is known that the optical reflectivity $R_{0}$ of metals decreases with increasing temperature [3], due to the lack of experimental data, it is assumed temperature-independent. In the case of the silicon substrate, the reflectivity of the solid is about $20 \%$ lower than the liquid (for near UV wavelength, $R_{0}\left(T<T_{m}\right) \simeq 0.6$ and $R_{0}\left(T \geq T_{m}\right) \simeq 0.75[\underline{5},[59,[60])$ and different temperature profiles are obtained if the temperature dependence of $R_{0}$ is taken into account, as illustrated in Fig. 3.3. In particular, when the melting point is reached, the higher reflectivity of the liquid phase reduces the absorbed laser energy, leading to a decrease in the maximum surface 




Figure 3.2: Time evolution of the surface temperature profiles (left) and the melting depths (right) of the iron and the aluminum substrates irradiated at $4 \mathrm{~J} / \mathrm{cm}^{2}$. The melting and the boiling points of each element are indicated.

temperature and in the melting time.



Figure 3.3: Comparison between the $\mathrm{Si}$ surface temperature profiles obtained using temperature-dependent (solid line) and temperature-independent (dashed line) reflectivities. 


\subsection{Phenomena occurring on the target surface}

In the previous sections, the temporal evolution of temperature profiles and the related phase transformations inside the irradiated substrates have been described, but important phenomena take place also on the surface of the target, as illustrated in the following sections.

\subsubsection{Vaporization}

According to the previous discussion of the heat equation, the laser beam can be absorbed by the substrate causing melting and vaporization. In the case of strong evaporation, typically at fluences of several $\mathrm{J} / \mathrm{cm}^{2}$ and nanosecond pulse duration, the more proper boundary condition at the target surface would be to include the velocity $v_{e v}$ of the evaporation front. Using Eq. (3.12) we can write [5]:

$$
v_{e v}=\frac{M}{\rho} j=\frac{p(T)}{\rho} / \sqrt{2 \pi R T / M} .
$$

In the reference frame attached to the liquid-vapor interface moving with velocity $v_{e v}$, the heat equation Eq. (3.2) becomes:

$$
\frac{\rho c_{p}(T)}{M}\left(\frac{\partial T(z, t)}{\partial t}-v_{e v} \frac{\partial T(z, t)}{\partial z}\right)=-\frac{\partial J_{\text {heat }}}{\partial z} .
$$

Both cases of stationary and non-stationary evaporations can be treated with proper approximations [5], but the correct treatment should consider: (i) the hydrodynamic motion of the evaporated material, (ii) the decrease of the vapor temperature due to its expansion and (iii) the backward flux of the evaporated species. The vaporized atoms/molecules leave the substrate at temperature $T_{s}$ with half-Maxwellian non-equilibrium velocity distribution (the velocity are initially in the direction normal to the target surface). Due to the collisions with other atoms/molecules, the vapor propagates with hydrodynamic speed $v_{v}$ and the velocity distribution becomes Maxwellian (i.e. in thermodynamical equilibrium) [61. The transformation from non-equilibrium to equilibrium distributions takes place in a thin layer of few mean free paths called Knudsen layer, as sketched in Fig. 3.4 . The detailed mathematical analysis of the transformation was performed by Anisimov [62] with a proper definition of the velocity distribution and using the conservation of mass, momentum and energy across the Knudsen layer. The results show that the temperature $T_{v}$ of the vapor beyond the Knudsen layer is lower than $T_{s}$, due to the partial transformation of thermal energy into kinetic energy of the expanding vapor plume. Besides, the number density of the vaporized species and the vapor pressure behind the Knudsen layer (subscript " $v "$ ) can be related to the same values within the layer (subscript " $s "$ ) as follows:

$$
\begin{aligned}
T_{v} & =T_{s}(1-0.33 \Pi) \\
N_{v} & =N_{s}\left(T_{s}\right) /(1+2.2 \Pi) \\
p_{v} & =N_{v} k_{B} T_{v}=p_{s}\left(T_{s}\right) \frac{1-0.33 \Pi}{1+2.2 \Pi},
\end{aligned}
$$






Figure 3.4: Irradiation geometry (top) and temperature profiles within the target and the ambient medium (bottom): effect of the Knudsen layer.

where the Mach number $\Pi$ determines the expansion velocity $v_{v}$ of the species beyond the Knudsen layer: in general $v_{v}=\Pi c_{s}$, where $c_{s}$ is the speed of sound. For a vapor expanding in vacuum, $\Pi=1$ [61], but if the laser irradiation takes place in a gaseous medium, $\Pi$ (with $0<\Pi<1$ ) must be calculated theoretically or measured experimentally [5].

\subsubsection{Recondensation}

Using Eqs. 3.24 and considering the vapor as an ideal gas, it can be verified that the vapor beyond the Knudsen layer is strongly supersaturated: in fact, $N_{v}=N_{s}\left(T_{s}\right) /(1+2.2 \Pi)>N_{s}\left(T_{v}\right)=p_{s}\left(T_{v}\right) / k_{B} T_{v}$. An example is reported in Fig. 3.5. The ratios $N_{v} / N_{s}\left(T_{v}\right)$ and $p_{v} / p_{s}\left(T_{s}\right)$ for an iron target at $T_{s}=4500 \mathrm{~K}$ are plotted as a function of the Mach number $\Pi$. The supersaturation is always present $\left(N_{v} / N_{s}\left(T_{v}\right)>1\right)$, and it is much stronger for high values of $\Pi$ (i.e. for low ambient pressures). Therefore, the recondensation of the evaporated species may start beyond the Knudsen layer. Besides, in the presence of a reactive atmosphere, the chemical reaction between the vapor and the ambient gas might lead to the formation and the subsequent condensation of chemical compounds. The pressure acting on the target surface is the pressure inside the Knudsen layer, that is the saturated vapor pressure $p_{s}$ at the temperature $T_{s}$, given by the Clausius-Clapeyron equation (Eq. (3.13)). For an Fe substrate at $T_{s}=4500 \mathrm{~K}$, we have $p_{s}\left(T_{s}\right) \simeq 10^{2}$ bar. The dramatic effect of the surface temperature on the saturated vapor pressure is illustrated in Fig. 3.6. the surface temperature profiles of the silicon target already shown in Fig. 3.3 have been used to compute the vapor pressure vs. time according to the Clausius-Clapeyron equation. A moderate increment of the surface temperature 


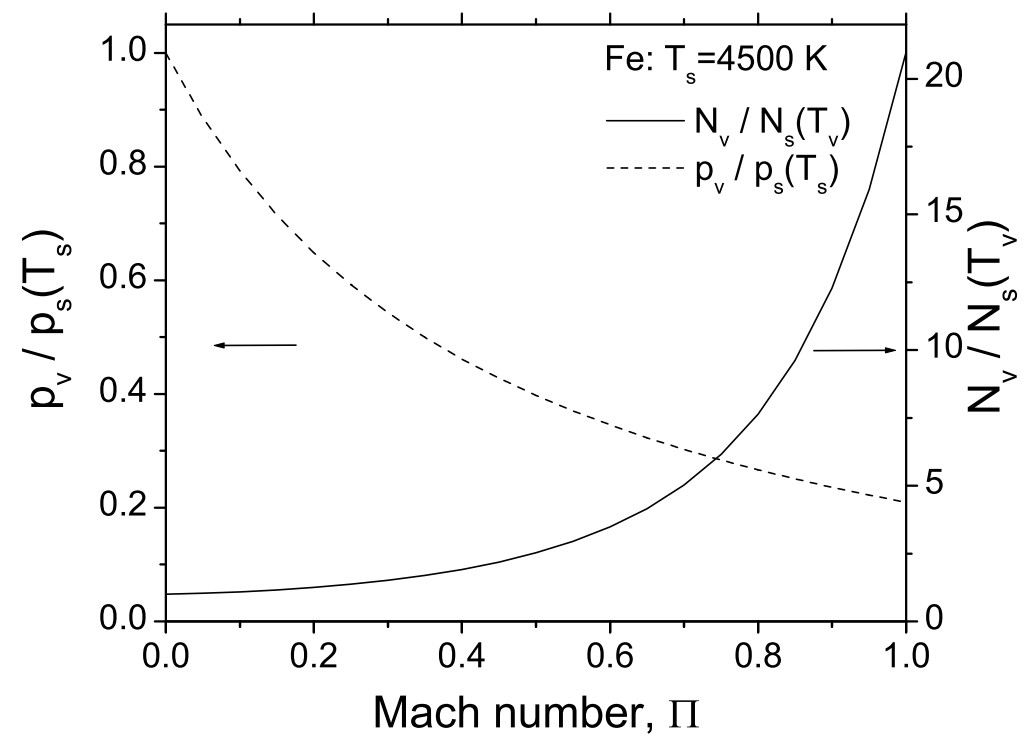

Figure 3.5: Ratios of the vapor pressures within and beyond the Knudsen layer (dashed line) and the densities of the vaporized species obtained from the Anisimov analysis and from ClausiusClapeyron equation (solid line) as a function of the Mach number.

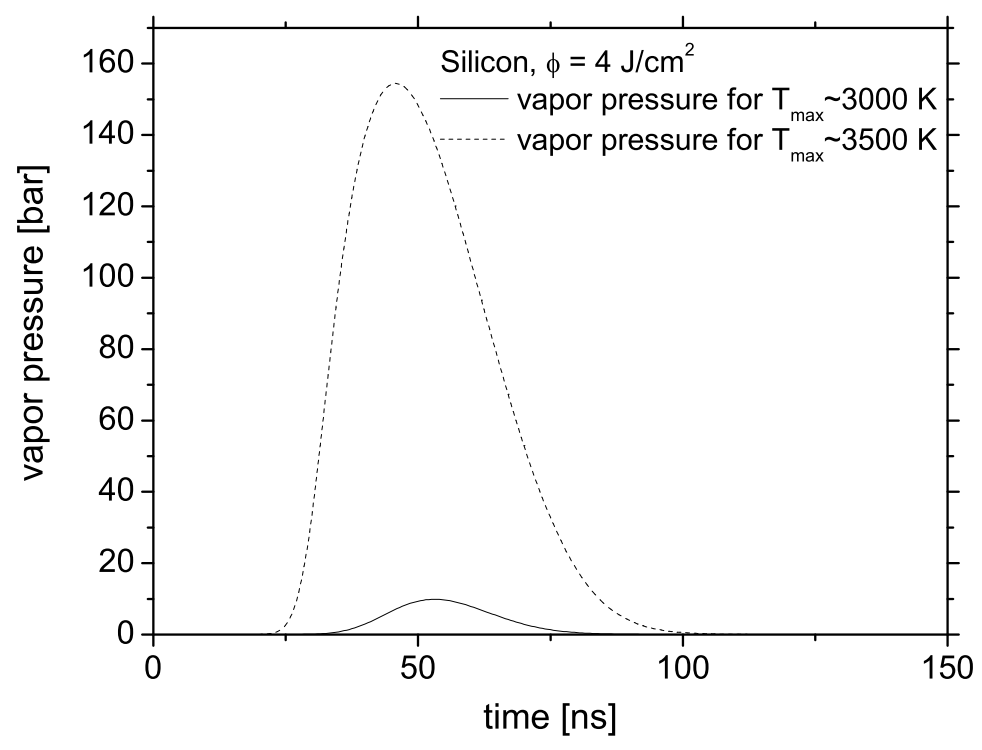

Figure 3.6: Influence of the Si surface temperature on the saturated vapor pressure (the temperature profiles shown in Fig. 3.3 have been used to calculate the vapor pressures).

from $3000 \mathrm{~K}$ to $3500 \mathrm{~K}$ leads to the enormous increase of the vapor pressure from $10 \mathrm{bar}$ to almost 160 bar. 


\subsubsection{Plasma formation}

When the laser intensity is high enough, the vapor or the ambient gas can become ionized, and properly described as a plasma. Within a gas at temperature $T_{g}$ the collisions between thermal electrons and vaporized species produce a certain degree of ionization given by the Saha equation [5]. The ionized gas strongly absorbs the laser radiation and expands within the laser beam channel, thus shielding the substrate from the laser light. The propagating plasma is generally termed laser-supported absorption wave (LSAW). If the LSAW moves with subsonic velocity with respect to the ambient medium, it is called laser-supported combustion wave (LSCW). As the laser intensity increases, the LSAW can exceed the sound speed becoming a laser-supported detonation wave (LSDW). The typical irradiance necessary to ionize a gas with a free propagating laser (i.e. without any target) is of the order of $10^{9}-10^{11} \mathrm{~W} / \mathrm{cm}^{2}$, but it can decrease by several orders of magnitude in front of a solid or liquid target [3, 5]. The theories of LSC and LSD waves have been developed in the '70 [63, 64, 65, 66] obtaining quantitative evaluations of the propagation velocity of the wave front and the pressure behind it. In the case of LSC wave, the laser-light is absorbed within the plasma and dissipated in the ambient medium via heat conduction and thermal radiation. The energy balance can be written as [66]:

$$
k^{e f f} \Delta T / d=d\left(\alpha_{p} I_{0}-J^{l o s s}\right),
$$

where $k^{e f f}$ is an effective thermal conductivity, $\alpha_{p}$ is the absorption coefficient of the plasma, $\Delta T$ is the temperature jump across the LSC wave, $d$ is the thickness of the wave front, $I_{0}$ is the laser irradiance and $J^{\text {loss }}$ is the volumetric energy loss $\left[\mathrm{J} / \mathrm{cm}^{3}\right]$ of the plasma, due to radiation/conduction. Using the heat equation, we obtain [66]:

$$
\frac{\rho_{p} c_{p}}{M_{p}} \frac{\partial T}{\partial t}=\frac{\rho_{p} c_{p}}{M_{p}} v_{L S C} \frac{\Delta T}{\Delta x}=v_{L S C} \frac{\rho_{p} c_{p}}{M_{p}} \frac{\Delta T}{d}=k^{e f f} \frac{\Delta T}{d^{2}}
$$

where $\rho_{p}, c_{p}$ and $M_{p}$ are the mass density, the molar specific heat and the molar mass of the heated gas, while $v_{L S C}$ is the velocity of the wave front. Using Eq. (3.25) in Eq. (3.26) to eliminate $d$, the LSCW front velocity becomes [66]:

$$
v_{L S C}=\frac{M_{p} k^{e f f}}{\rho_{p} c_{p}} \sqrt{\frac{\alpha_{p} I_{0}-J^{\text {loss }}}{k^{e f f} \Delta T}} .
$$

For intense laser beams $\left(I_{0} \gg J^{\text {loss }} / \alpha_{p}\right)$, we have $v_{L S C} \propto I_{0}^{1 / 2}$ and typical values of $10^{3}$ to $10^{4} \mathrm{~cm} / \mathrm{s}[3]$. On the other hand, if $I_{0} \approx J^{\text {loss }} / \alpha_{p}$, we have $v_{L S C} \approx 0$ and the LSCW becomes a stationary wave called plasmatron [67, 68]. If the velocity of the propagating wave exceeds the sound velocity of the medium, a supersonic LSD wave is produced. Treating such a wave as a hydrodynamic discontinuity, and using the conservation of mass, momentum and energy, its velocity can be estimated as [63]:

$$
v_{L S D}=\left[2\left(\gamma^{2}-1\right) I_{0} / \rho_{0}\right]^{1 / 3} \propto I_{0}^{1 / 3},
$$

where $\gamma$ is the adiabatic coefficient and $\rho_{0}$ is the mass density of the ambient gas. The gas pressure behind the wave is [63]:

$$
p_{L S D}=\frac{\rho_{0} v_{L S D}^{2}}{\gamma+1} \propto I_{0}^{2 / 3}
$$


For a laser irradiance $I_{0}=72 \mathrm{MW} / \mathrm{cm}^{2}$ (i.e. our excimer laser, see next chapter) and considering air in standard conditions $\left(\rho_{0} \approx 1.3 \mathrm{~kg} / \mathrm{m}^{3}\right.$ and $\left.\gamma \approx 7 / 5\right)$ as a medium, we obtain $v_{L S D} \approx 10^{6} \mathrm{~cm} / \mathrm{s}$ and $p_{L S D} \approx 5 \times 10^{2}$ bar. It is now clear that in both cases of pure vaporization and plasma formation, the pressure acting on the target surface is of the order of $10^{2}$ bar. As already mentioned, this effect rises the boiling point of the target, and the melt can be heated well above $T_{b}$. Therefore, the latent heat of evaporation $L_{e v}$ does not play the same role of heat sink as the latent heat of melting $L_{m}$, and Eqs. 3.10) are unnecessary for the liquid-vapor phase transition. 


\section{Chapter 4}

\section{Experimental methods}

The following sections describe the experimental details of the samples preparation, samples treatment and analyses via ion beam techniques (Rutherford Backscattering Spectrometry, Resonant Nuclear Reaction Analysis), X-ray techniques (X-ray Absorption Spectroscopy, X-ray Diffraction), Mössbauer Spectroscopy and nanoindentation hardness measurements. Each technique will be described briefly. For more detailed explanations, the specific literature will be reported.

\subsection{Samples preparation and laser treatments}

Armco iron (purity 99.85\%) and aluminum (purity 99.9\%) rods have been cut into slices of 1-1.5 mm thickness, mechanically polished with $\mathrm{SiC}$ grinding paper $(1200,2400,4000$ mesh) and then with $1 \mu \mathrm{m}$ diamond paste in order to obtain mirror-like metal surfaces. Single crystalline, boron-doped wafer, $\langle 100\rangle$ and $<111>$ oriented, $0.5 \mathrm{~mm}$ thick, have been used as silicon substrates without any further treatment. The laser irradiations have been performed with a Siemens XP $2020 \mathrm{XeCl}$ excimer laser: its basic characteristics are

reported in Table 4.1. The samples were placed inside a chamber equipped with a quartz

Table 4.1: Basic features of the Siemens XP 2020 excimer laser.

\begin{tabular}{lr}
\hline \hline Parameter & Value \\
\hline Laser gas & XeCl \\
Wavelength $\lambda[\mathrm{nm}]$ & 308 \\
Maximum pulse energy $E_{\max }[\mathrm{J}]$ & 2 \\
Pulse duration $t_{p}$ FWHM $[\mathrm{ns}]$ & 55 \\
Maximum pulse frequency $f_{p}[\mathrm{~Hz}]$ & 10 \\
\hline \hline
\end{tabular}

window and mounted on a X-Y stage. In order to reduce the oxygen contaminations, the chamber was evacuated to a residual pressure $<10^{-8}$ bar, and then filled with the desired gas (typically at pressures between 0.1 bar and 10 bar). Natural nitrogen (purity 99.999\%) and methane (purity 99.5\%) have been used for most of the treatments, but 
in some cases the use of isotopic gas was required: ${ }^{15} \mathrm{~N}$ enriched nitrogen gas ( $\sim 18 \%$ enrichment, i.e. 49 times larger than in the natural gas) was used for the detailed analysis of the nitrogen depth profiles by means of Resonant Nuclear Reaction Analysis (more details on the isotopic experiments will be found in the next chapter). The laser beam was focused through a flat-concave quartz lens with focal length of $190 \mathrm{~mm}$, obtaining the so-called raw beam. The distance between the lens and the sample was adjusted in order to irradiate an area $A$ of about $25 \mathrm{~mm}^{2}$. Since the energy profile obtained with this lens is quite inhomogeneous, leading to a strong convection on the irradiated targets, most of the laser treatments have been performed employing a fly-eye homogenizer lens with focal length of $140 \mathrm{~mm}$. At the optimal distance between the homogenizer and the sample, a square spot of area $A=5 \times 5 \mathrm{~mm}^{2}$ is produced, with minimized surface roughness compared to the raw beam [31]. The laser energy $E$ was measured with a pyroelectric joulemeter and the energy fluence $\phi$ was determined as $\phi=E / A$ (the area of the laser spot was estimated experimentally with the help of a surface profiler). Since the area $A$ was fixed, the laser fluence was varied changing the laser energy $E$ by means of an additional attenuator (a semitransparent mirror) placed between the laser and the lens: varying the angle between the normal to the mirror and the laser direction, the amount of reflected/transmitted light could be easily modified. The laser experimental setup is sketched in Fig. 4.1. Two kinds of laser treatments have been performed: with the single

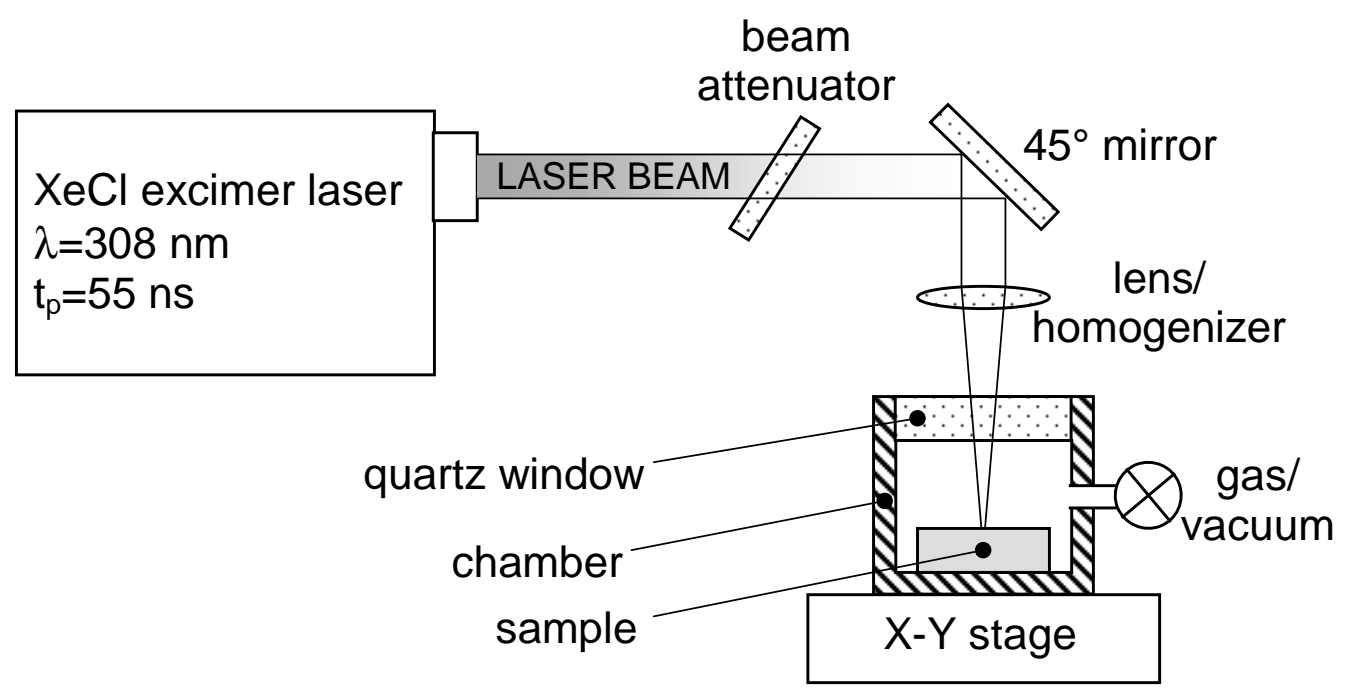

Figure 4.1: Sketch of the laser irradiation experimental setup.

spot irradiation, the same spot on the target was irradiated with the desired number of laser shots (typically from 1 to $\sim 500$ ); with the meandering treatment, the sample was shifted after each laser pulse in such a way that the displacement was a fraction of the spot size. The pictorial explanation is reported in Fig. 4.2. if $a$ is the size of the laser spot, the sample shift $\Delta x=a / n$ in the $x$ direction and $\Delta y=a / m$ in the $y$ direction lead to the meander treatment termed $n \times m$, i.e. each square of area $a^{2}$ on the sample surface is irradiated $n \cdot m$ times in toto. 


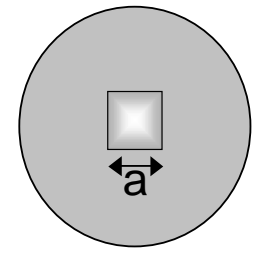

single spot



meandering

Figure 4.2: Sketch of the single spot and the meandering treatments.

\subsection{Rutherford Backscattering Spectrometry}

The Rutherford Backscattering Spectrometry (RBS) is based on the elastic scattering of an ion beam ( $\alpha$ particles with $\mathrm{MeV}$ energy) at the target nuclei. The detection and energetic analysis of the backscattered particles allow to measure the elemental depth profile of the target. The principle of the technique is sketched in Fig. 4.3. All RBS

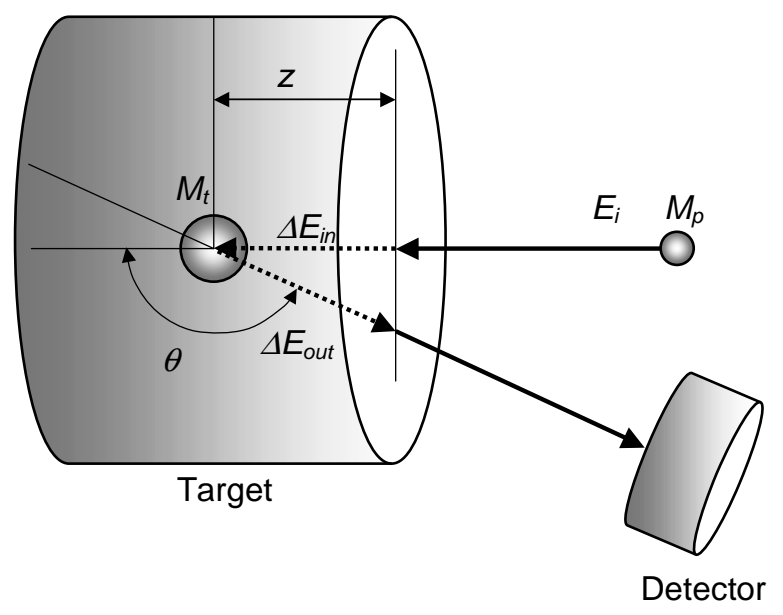

Figure 4.3: Sketch of the RBS experiment.

measurements have been carried out at the IONAS accelerator facility in Göttingen [69]. The $\alpha$ particle beam $\left(M_{p}=4 \mathrm{amu}\right)$ with energy $E_{i}$ of $0.9 \mathrm{MeV}$ is scattered at the target element of mass $M_{t}$ and detected at a scattering angle $\theta$ of $165^{\circ}$. The energy $E_{i}$ of the incident beam is reduced after the collision by the so called kinematic factor $K\left(M_{t}, \theta\right)$ as follows [70]:

$$
E_{s}=K\left(M_{t}, \theta\right) E_{i}=\left(\frac{M_{p} \cos \theta+\sqrt{M_{t}^{2}-M_{p}^{2} \sin ^{2} \theta}}{M_{p}+M_{t}}\right)^{2} E_{i}
$$

If the scattering process takes place at the surface of the probe, the $\alpha$ beam is detected with energy $E_{s}$ as given by the previous equation. If the scattering process takes place at 
a depth $z$ from the surface of the sample, the $\alpha$ beam will loose an amount of energy $\Delta E_{\text {in }}$ penetrating the sample due to the stopping power of the target. After the scattering, the beam with energy $K\left(E_{i}-\Delta E_{i n}\right)$ will travel a distance $z / \cos (\pi-\theta)$ in the direction of the target surface, loosing an amount of energy $\Delta E_{\text {out }}$, and the $\alpha$ particle will be detected with final energy $E_{s}(z)$ given by:

$$
E_{s}(z)=K\left(E_{i}-\Delta E_{\text {in }}\right)-\Delta E_{\text {out }}=K\left(E_{i}-\int_{0}^{z} \frac{d E}{d z} d z\right)-\int_{0}^{\frac{z}{\cos (\pi-\theta)}} \frac{d E}{d z} d z .
$$

The quantity $\frac{d E}{d z}$ is called stopping power and it represents the energy loss per distance traveled in the target. It is correlated to the stopping cross section $\varepsilon$, defined as the energy loss per atom per areal density of the target (usual units $\mathrm{eV} / 10^{15}$ atoms $\mathrm{cm}^{-2}$ ) [70]:

$$
\varepsilon=\frac{1}{N} \frac{d E}{d z}
$$

where $N$ is the atomic density of the target. Since the stopping cross section of a $\alpha$ particle beam is tabulated as function of its energy for all the elements of the periodic table, it can be calculated for any compound $\mathrm{A}_{x} \mathrm{~B}_{y}$ according to the Bragg's rule [71]:

$$
\varepsilon_{A_{x} B_{y}}=x \varepsilon_{A}+y \varepsilon_{B}
$$

with $\varepsilon_{A}$ and $\varepsilon_{B}$ being the stopping cross sections of the pure elements $\mathrm{A}$ and $\mathrm{B}$. The corresponding stopping power to be used in Eq. (4.2) is then:

$$
\left(\frac{d E}{d z}\right)_{A_{x} B_{y}}=N_{A_{x} B_{y}}\left(x \varepsilon_{A}+y \varepsilon_{B}\right)
$$

where $N_{A_{x} B_{y}}$ is the molecular density of the compound $\mathrm{A}_{x} \mathrm{~B}_{y}$. The probability to detect a backscattered $\alpha$ particle is proportional to the total cross section $\sigma$ averaged over the solid angle $\Omega$ of the detector:

$$
\sigma\left(E_{i}, \theta\right)=\frac{1}{\Omega} \int \frac{d \sigma}{d \Omega} d \Omega
$$

The quantity $\frac{d \sigma}{d \Omega}$ is called differential cross section and it depends on the incident energy $E_{i}$ of the beam, its scattering angle $\theta$, the masses and the nuclear charges of the atoms involved in the scattering [70]:

$$
\frac{d \sigma}{d \Omega}=\left(\frac{Z_{p} Z_{t} e^{2}}{4 E_{i}}\right)^{2} \frac{4}{\sin ^{4} \theta} \frac{\left[\sqrt{1-\left(M_{p} / M_{t}\right)^{2} \sin ^{2} \theta}+\cos \theta\right]^{2}}{\sqrt{1-\left(M_{p} / M_{t}\right)^{2} \sin ^{2} \theta}} .
$$

Since the target has typically atomic species much heavier than the $\alpha$ particles $\left(M_{p}<<M_{t}\right)$ the previous equation can be simplified as follows:

$$
\frac{d \sigma}{d \Omega}=\left(\frac{Z_{p} Z_{t} e^{2}}{4 E_{i}}\right)^{2} \frac{1}{\sin ^{4}(\theta / 2)} .
$$

For a $\alpha$ beam impinging at normal incidence on a uniform target, the total number $H$ of detected particles can be written as [72]:

$$
H=\sigma \Omega Q \cdot N d,
$$


where $Q$ is the total number of particles hitting the sample, $N d$ is the number of target atoms per unit area and $\sigma$ is calculated from Eqs. (4.6) and (4.8). When $\sigma$ and $\Omega$ are known, and the numbers of detected and incident particles are measured, the number of atom per unit area $N d$ can be determined. Besides, if the atomic density $N$ of the target is known, the physical thickness $d$ can be found. For a multielemental compound $\mathrm{A}_{x} \mathrm{~B}_{y}$, the extension of Eq. (4.9) is straightforward, since

$$
H_{A_{x} B_{y}}=H_{A}+H_{B}
$$

where $H_{A}=\sigma_{A} \Omega Q \cdot N_{A} d_{A}$ and $H_{B}=\sigma_{B} \Omega Q \cdot N_{B} d_{B}$. Being $N_{A}=x N_{A_{x} B_{y}}$ and $N_{B}=y N_{A_{x} B_{y}}$, form Eq. 4.10) we obtain [72]:

$$
\frac{H_{A}}{H_{B}}=\frac{\sigma_{A} \Omega Q x N_{A_{x} B_{y}} d}{\sigma_{B} \Omega Q y N_{A_{x} B_{y}} d}=\frac{\sigma_{A} x}{\sigma_{B} y},
$$

and the stoichiometric ratio of the compound is readily found:

$$
\frac{x}{y}=\frac{H_{A} \sigma_{B}}{H_{B} \sigma_{A}}
$$

Eq. 4.9) and Eq. (4.12) reveal how the RBS is a suitable technique for measuring the depth profile and the elemental composition of the target. An example of a RBS spectrum is reported in Fig. 4.4. A thin Fe film $(90 \mathrm{~nm})$ deposited by Physical Vapor Deposition (PVD) on a Si substrate has been analyzed by RBS with a $900 \mathrm{keV} \mathrm{He}{ }^{++}$beam. The

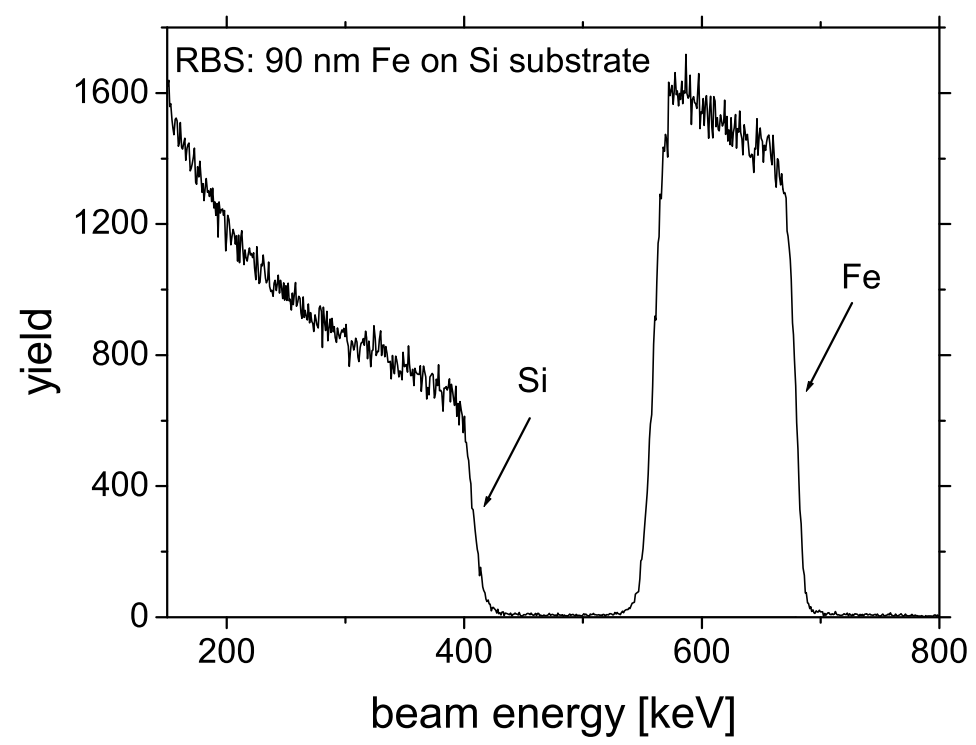

Figure 4.4: RBS spectrum of a thin Fe film (90 nm) deposited on a Si substrate. The arrows indicate the energy positions of the Fe and the Si surface/interface.

depth resolution of RBS is limited by: (i) the energy width of the incident $\alpha$ beam, (ii) the detector resolution and (iii) the energy straggling. The first two contributions are instrumental effects. The beam energy width at the IONAS accelerator has a gaussian 
shape with width $\omega_{\text {beam }}$ depending on the energy and the charge of the accelerated particle: for a $0.9 \mathrm{MeV} \alpha$ beam we have $\omega_{\text {beam }}=79 \mathrm{eV}$ [69], while the typical energy resolution $\omega_{\text {res }}$ of a solid state silicon detector is of the order of $12 \mathrm{keV}$. On the other hand, the beam straggling is a physical effect. As the charged particles traverse the target, the statistical fluctuations of the collision processes produce a spreading of the beam energy. Bohr [73] formulated the beam straggling in the limit of high ion velocity where the energy loss is mostly electronic:

$$
\omega_{B}^{2}\left[\mathrm{keV}^{2}\right]=0.26 Z_{p}^{2} Z_{t} N d\left[10^{18} \text { at. } / \mathrm{cm}^{2}\right] .
$$

$\omega_{B}^{2}$ is the variance of the energy loss fluctuations. Lindhard and Sharff [74] extended the validity of Eq. (4.13) to beam energies $E$ [keV/amu] below $75 Z_{t}$ :

$$
\omega_{L S}^{2}= \begin{cases}f(E) \omega_{B}^{2} & \text { if } E<75 Z_{t} \\ \omega_{B}^{2} & \text { if } E \geq 75 Z_{t}\end{cases}
$$

with $f(E)=0.136\left(E / Z_{t}\right)^{1 / 2}-0.000064\left(E / Z_{t}\right)^{3 / 2}$. The total beam energy spreading can be quantified as:

$$
\omega_{\text {tot }}=\sqrt{\omega_{\text {beam }}^{2}+\omega_{L S}^{2}+\omega_{\text {res }}^{2}}
$$

and it can be related to the depth resolution $\Delta z$ using the definition of $\varepsilon$ (Eq. (4.3)):

$$
\Delta z=\omega_{t o t} / \varepsilon N
$$

The RBS data analysis has been performed with the RUMP code [75, that enables to take into account the stopping cross section, the beam straggling and the detector resolution.

\subsection{Resonant Nuclear Reaction Analysis}

The Resonant Nuclear Reaction Analysis (RNRA) is a powerful tool for chemical depth profiling, due to its sensitivity to specific isotopes. The principle of the technique is sketched in Fig. 4.5. When the proton beam hits the target containing the isotope of

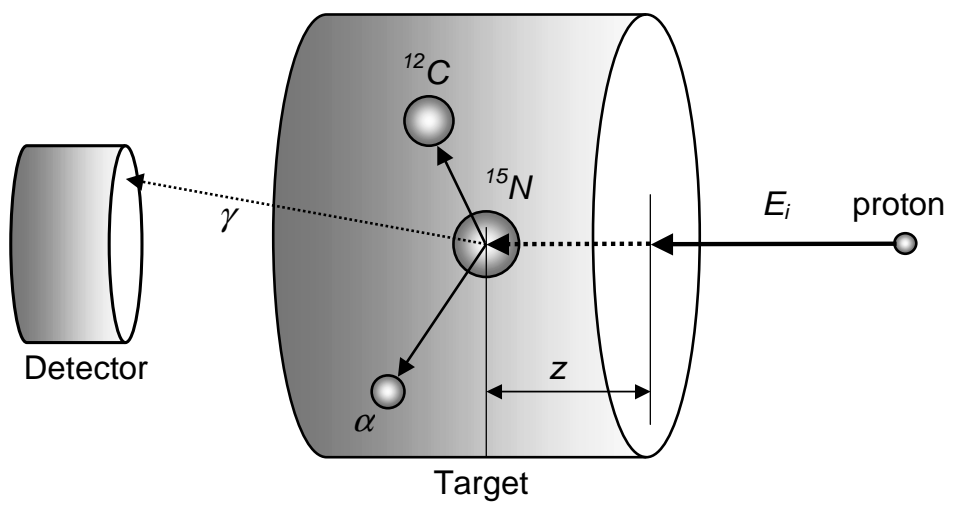

Figure 4.5: Sketch of the ${ }^{15} \mathrm{~N}(\mathrm{p}, \alpha \gamma){ }^{12} \mathrm{C}$ RNRA experiment.

interest, the nuclear reaction can occur, leading to the emission of $\gamma$-rays. The photon yield can be used to estimate the average concentration of the corresponding element. If the energy of the analyzing beam corresponds to the energy $E_{r}$ of a narrow resonance, the 
reaction takes place at the surface of the target. At a higher beam energy, the resonant energy $E_{r}$ is reached at a certain depth $z$ in the target, when the proton has lost the exceeding energy. The $\gamma$-ray yield as a function of the incident beam energy $E_{i}$ (with $E_{i} \geq E_{r}$ ) resembles the concentration depth profile of the isotope. In practice, the yield can be converted into atomic concentration with the use of a calibration sample, and the energy can be converted into depth when the stopping power of the target is known. The nuclear reaction of interest in the present work is ${ }^{15} \mathrm{~N}(\mathrm{p}, \alpha \gamma){ }^{12} \mathrm{C}$ that has been used to measure the nitrogen depth profiles in the laser-irradiated substrates. The main physical properties of the reaction are reported in Table 4.2. In the generic sample of stoichiometry

Table 4.2: Main physical properties of the ${ }^{15} \mathrm{~N}(\mathrm{p}, \alpha \gamma){ }^{12} \mathrm{C}$ nuclear reaction [76].

\begin{tabular}{ccccc}
\hline \hline Reaction & $\begin{array}{c}\text { Resonance energy } \\
{[\mathrm{keV}]}\end{array}$ & $\begin{array}{c}\gamma \text {-ray energy } \\
{[\mathrm{MeV}]}\end{array}$ & $\begin{array}{c}\text { Cross section } \\
{[\mathrm{mb}]}\end{array}$ & $\begin{array}{c}\text { Width } \\
{[\mathrm{keV}]}\end{array}$ \\
\hline${ }^{15} \mathrm{~N}(\mathrm{p}, \alpha \gamma){ }^{12} \mathrm{C}$ & 429.6 & $4.43(100 \%)$ & 300 & 0.124 \\
\hline \hline
\end{tabular}

$A_{f} B_{1-f}$, the element to be profiled is $A$ and its atomic fraction is $f$ (with $0 \leq f \leq 1$ ). The normalized $\gamma$-ray yield $Y_{Q}$ (i.e. the yield $Y$ divided by the number of incident protons $Q$ ) of the investigated target is compared with the normalized yield $Y_{\text {cal }}$ of the calibration sample containing the same isotope of interest in a known fraction with a known depth profile (a TiN film deposited by magnetron sputtering on a Si substrate has been used). In general, the following relation holds [77]:

$$
\frac{f_{\text {cal }}}{Y_{\text {cal }} \varepsilon_{\text {cal }}}=\frac{f}{Y_{Q} \varepsilon}
$$

where $f_{c a l}$ is the atomic fraction of the element $\mathrm{A}$ in the calibration sample and $\varepsilon_{c a l}$ is the stopping cross section of the proton beam. Since the atomic fraction $f_{c a l}$ is known, $\varepsilon_{c a l}$ can be calculated with the Bragg's rule (see Eq. (4.4)). The stopping cross section in the sample under investigation can be computed as:

$$
\varepsilon=f \varepsilon_{A}+(1-f) \varepsilon_{B}=f\left(\varepsilon_{A}-\varepsilon_{B}\right)+\varepsilon_{B},
$$

with $\varepsilon_{A}$ and $\varepsilon_{B}$ being the stopping cross sections of the pure elements $A$ and $B$. Inserting Eq. (4.18) in Eq. (4.17) we obtain:

$$
\frac{f_{c a l}}{Y_{c a l} \varepsilon_{c a l}}=\frac{f}{Y_{Q}\left[f\left(\varepsilon_{A}-\varepsilon_{B}\right)+\varepsilon_{B}\right]},
$$

and solving with respect to $f$, the atomic fraction of the element $A$ as a function of the measured $\gamma$-ray yield $Y_{Q}$ becomes:

$$
f=\frac{f_{c a l} Y_{Q} \varepsilon_{B}}{Y_{c a l} \varepsilon_{c a l}+f_{c a l} Y_{Q}\left(\varepsilon_{B}-\varepsilon_{A}\right)} .
$$

For non homogeneous specimens, the atomic fraction $f$ is a function of the depth $z$, i.e. $f=f(z)$. In order to have the concentration depth profile, $Y_{Q}$ (and therefore the atomic fraction $f$ ) is measured at various energies $E \geq E_{r}$ (i.e. various depths):

$$
f(E)=\frac{f_{c a l} Y_{Q}(E) \varepsilon_{B}\left(E_{r}\right)}{Y_{c a l} \varepsilon_{c a l}\left(E_{r}\right)+f_{c a l} Y_{Q}(E)\left[\varepsilon_{B}\left(E_{r}\right)-\varepsilon_{A}\left(E_{r}\right)\right]} .
$$


Since the calibration sample is homogeneous, $f_{\text {cal }}$ and $Y_{\text {cal }}$ are independent of the beam energy. The energy scale is then converted to depth scale $z$ in $\left.10^{15} \mathrm{at} . / \mathrm{cm}^{2}\right]$ using the relation (see also Eq. (4.3)):

$$
z=\int_{E_{r}}^{E} \frac{d E^{\prime}}{\varepsilon\left(E^{\prime}\right)}=\int_{E_{r}}^{E} \frac{d E^{\prime}}{f\left(E^{\prime}\right)\left[\varepsilon_{A}\left(E^{\prime}\right)-\varepsilon_{B}\left(E^{\prime}\right)\right]+\varepsilon_{B}\left(E^{\prime}\right)},
$$

where Eq. (4.18) has been used to evaluate the stopping cross section in the investigated sample. The stopping cross section of a proton beam in $\mathrm{N}, \mathrm{Al}$ and $\mathrm{Fe}$ are reported as an example in Fig. 4.6 in the energy range 10-1000 keV. In practice, the $\gamma$-ray yield

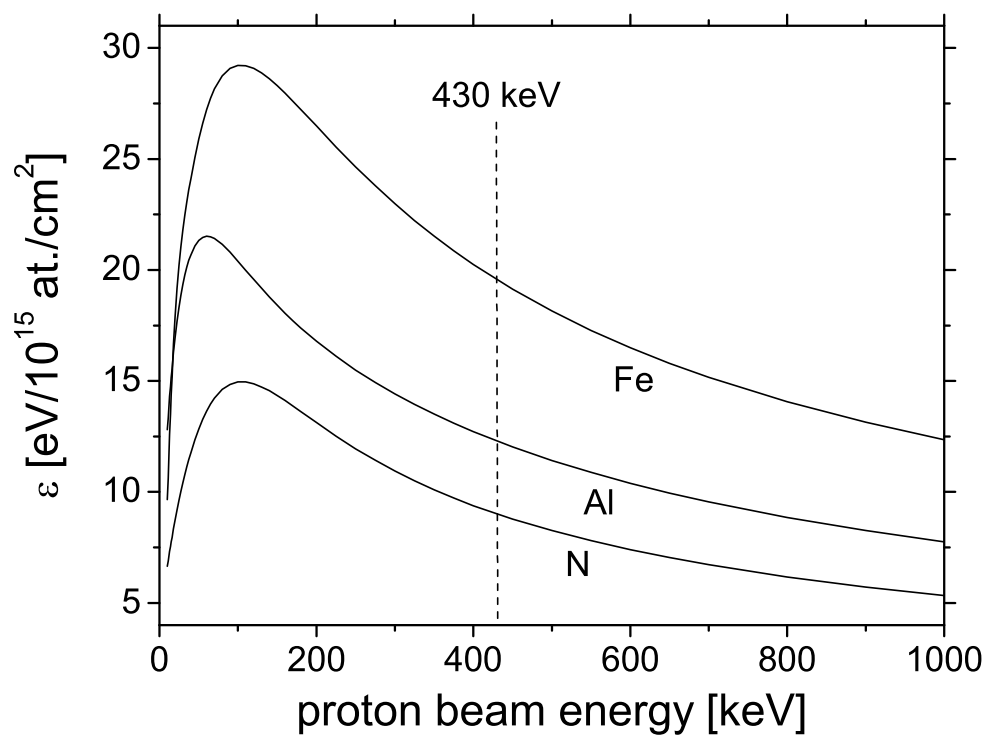

Figure 4.6: Stopping cross sections of a proton beam with 10-1000 keV energy in pure Fe, $\mathrm{Al}$ and $\mathrm{N}$ (gas). The resonance energy of the ${ }^{15} \mathrm{~N}(\mathrm{p}, \alpha \gamma){ }^{12} \mathrm{C}$ reaction is marked by the vertical dashed line.

is measured at discrete energies $E_{k}$ (with $E_{k} \geq E_{r}$ ) and the integral in Eq. 4.22) is calculated numerically with the trapezoid rule:

$$
z_{k}=\sum_{j=1}^{k} \frac{\left(E_{j+1}-E_{j}\right)}{2}\left(\frac{1}{\varepsilon\left(E_{j+1}\right)}+\frac{1}{\varepsilon\left(E_{j}\right)}\right)
$$

where $E_{1}=E_{\text {res }}$. Finally, the depth scale can be converted from $10^{15}$ at. $/ \mathrm{cm}^{2}$ to nanometers if the atomic density $N\left[\mathrm{at} . / \mathrm{cm}^{3}\right]$ of the sample is known as a function of $f$ :

$$
z[\mathrm{~nm}]=10^{7} z\left[10^{15} \mathrm{at} . / \mathrm{cm}^{2}\right] / N(f)\left[\mathrm{at} . / \mathrm{cm}^{3}\right] .
$$

Eqs. 4.18 to 4.24) can be easily implemented in a spreadsheet and the $\gamma$-ray yield vs. beam energy $Y_{Q}(E)$ can be transformed into atomic concentration vs. depth $f(z)$, as shown in Fig. 4.7. A number of factors can affect the depth resolution of the RNRA: (i) the width of the nuclear resonance, (ii) the energy width of the proton beam, (iii) the 

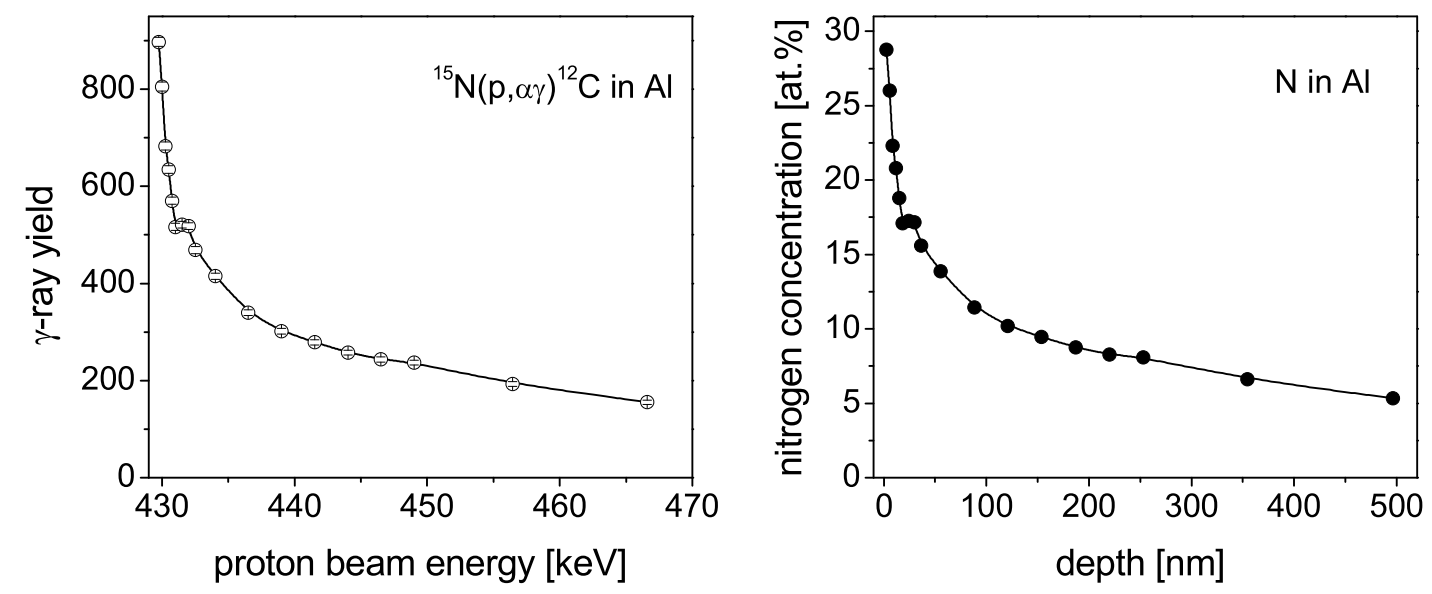

Figure 4.7: Conversion of the $\gamma$-rays yield vs. beam energy (left) to nitrogen concentration in aluminum vs. depth (right) according to the procedure described by Eqs. 44.18)- 4.24).

Doppler broadening of the target nuclei and (iv) the proton beam straggling. The cross section width $\Gamma$ of a given resonance is described by the Breit-Wigner formula:

$$
\sigma(E)=\frac{\sigma_{0} \Gamma^{2}}{\left(E-E_{r}\right)^{2}+\Gamma^{2} / 4}
$$

The energy resolution of a $430 \mathrm{keV}$ proton beam at the IONAS accelerator is $\omega_{\text {beam }}=36 \mathrm{eV}$ [69]. The Doppler broadening is due to the vibrations of the target atoms and it follows a gaussian law with width $\omega_{D}$ given by [78]:

$$
\omega_{D}=2.355 \sqrt{2 M_{p} E k_{B} T / M_{t}}
$$

where $M_{p}$ and $E$ are the atomic mass and the energy of the beam, respectively, $M_{t}$ is the average atomic mass of the target, $T$ is the temperature and $k_{B}$ is the Boltzmann constant. During the measurement the sample is typically cooled to liquid nitrogen temperature $(\sim 80 \mathrm{~K})$ and the value of $\omega_{D}$ is of the order of $28 \mathrm{eV}$. The energy straggling $\omega_{L S}$ of the proton beam can be described by the Lindhard-Sharff model as already discussed in the previous section (see Eq. (4.14)). The total energy spreading of the proton beam is then:

$$
\omega_{t o t}=\sqrt{\Gamma^{2}+\omega_{b e a m}^{2}+\omega_{D}^{2}+\omega_{L S}^{2}}
$$

and the depth resolution $\Delta z$ can be written as (see also Eq. 4.16)):

$$
\Delta z=\omega_{t o t} / \varepsilon\left(E_{r}\right) N(f)
$$

The values of $\Delta z$ for a $430 \mathrm{keV}$ proton beam in pure Fe and pure $\mathrm{Al}$ targets are reported in Fig. 4.8 as an example. 


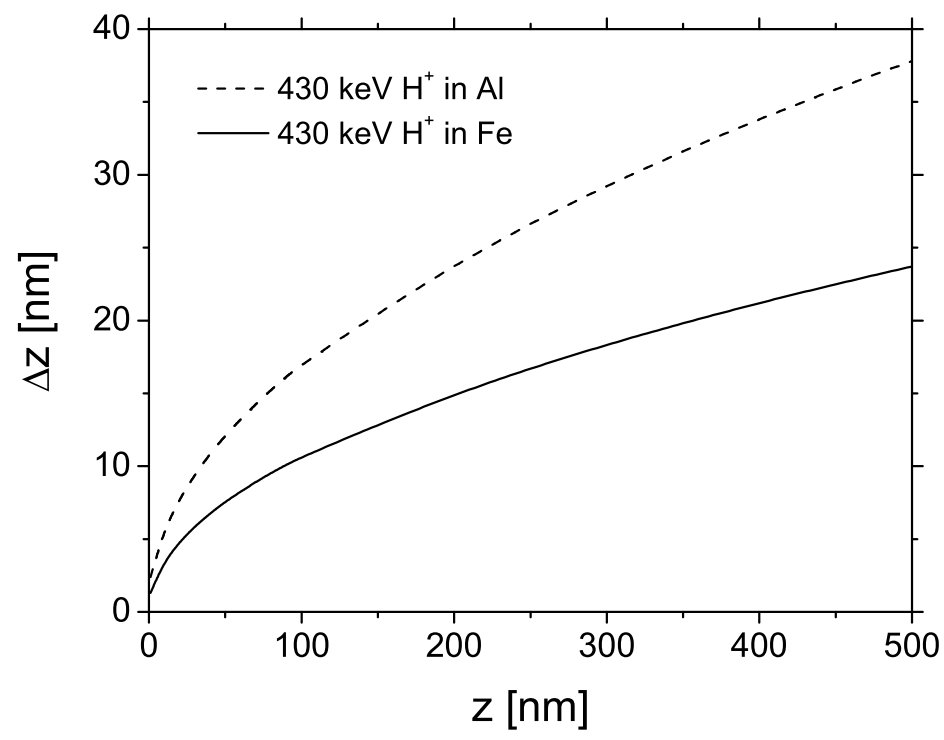

Figure 4.8: Depth resolution of a $430 \mathrm{keV}$ proton beam traversing an aluminum (dashed line) and an iron (solid line) target.

\subsection{Mössbauer Spectroscopy}

Mössbauer Spectroscopy is based on the resonant absorption of nuclear $\gamma$-rays without thermal broadening and without energy loss due to the recoil of the absorbing and the emitting nuclei [79]. An excited nucleus will emit $\gamma$-ray with an energy distribution given by the Breit-Wigner law:

$$
\sigma(E)=\frac{\sigma_{0} \Gamma^{2}}{\left(E-E_{0}\right)^{2}+\Gamma^{2} / 4},
$$

where $E_{0}$ is the transition energy $\left(14.4 \mathrm{keV}\right.$ for $\left.{ }^{57} \mathrm{Fe}\right)$ and $\Gamma$ is the resonance width $\left(4.7 \times 10^{-9} \mathrm{eV}\right.$ for $\left.{ }^{57} \mathrm{Fe}\right)$. Since the momentum of a $\gamma$-ray with energy $E_{0}$ is $E_{0} / c$, a free emitting atom of mass $M$ would recoil with energy $E_{r e c}=E_{0}^{2} / 2 M c^{2}$. Nevertheless, if the nucleus is bound in a solid, there is a non-zero probability that the recoil is transferred to the whole solid with mass of the order of $10^{20} \mathrm{M}$, making the recoil energy negligible. This is the essence of the Mössbauer effect [80, 81, 82]. The recoil-free fraction $f_{D}$ of a nucleus emitting or absorbing a $\gamma$-ray is given by the Debye-Waller factor [79]:

$$
f_{D W}=\exp \left[\frac{-6 E_{0}}{k_{B} \Theta}\left(\frac{1}{4}+\frac{T^{2}}{\Theta^{2}} \int_{0}^{\Theta / T} \frac{x d x}{e^{x}-1}\right)\right],
$$

with $\Theta$ being the Debye temperature of the sample and $T$ its local temperature. The $\gamma$ ray energy of a typical Mössbauer transition is so well defined that its Heisemberg width corresponds to an energy shift produced by a Doppler velocity of $1 \mathrm{~mm} / \mathrm{s}$. Therefore, the transition energy of a $\gamma$-ray source and a $\gamma$-ray absorber can precisely match if the energy is modulated by Doppler effect. This turns the Mössbauer effect into spectroscopy. A Mössbauer spectrum consists of a series of $\gamma$-ray yield measurements at different relative velocities (i.e. energies) between the source and the absorber. The high energy 
resolution allows the detailed measurement of the hyperfine interaction, that is the modification of the nuclear energy levels due to the electric and magnetic fields at the nuclear site. The electrostatic energy due to the interaction between the nuclear charges and the surrounding electrons can be written as [79, 83]:

$$
W=\int \rho(\mathbf{r}) \phi(\mathbf{r}) d V
$$

where $\rho(\mathbf{r})$ and $\phi(\mathbf{r})$ are the nuclear charge distribution and electronic potential, respectively. Due to the infinitesimal dimensions of the nucleus, $\phi(\mathbf{r})$ can be written as a Taylor series:

$$
\phi(\mathbf{r})=\phi(\mathbf{0})+\left.\sum_{i=1}^{3} \frac{\partial \phi}{\partial x_{i}}\right|_{\mathbf{0}} x_{i}+\left.\frac{1}{2} \sum_{i, j=1}^{3} \frac{\partial^{2} \phi}{\partial x_{i} \partial x_{j}}\right|_{\mathbf{0}} x_{i} x_{j} .
$$

Substituting Eq. (4.32) in Eq. (4.31) with a proper arrangement of all terms and a suitable definition of the reference frame [79], the electrostatic energy becomes:

$$
W=\phi(\mathbf{0}) Z e+\frac{1}{6} \sum_{\alpha=1}^{3} \phi_{\alpha \alpha} \int \rho(\mathbf{r}) r^{2} d V+\frac{1}{6} \sum_{\alpha=1}^{3} \phi_{\alpha \alpha} \int \rho(\mathbf{r})\left(3 x_{\alpha}^{2}-r^{2}\right) d V,
$$

with $\phi_{\alpha \alpha}$ being the diagonal terms of the electric field gradient (EFG) tensor and $Z$ the nuclear charge (i.e. the atomic number). The first two terms in the r.h.s. of Eq. (4.33) determine the interaction energy of the so-called isomer shift (IS):

$$
E_{I S}=\phi(\mathbf{0}) Z e+\frac{1}{6} \sum_{\alpha=1}^{3} \phi_{\alpha \alpha} \int \rho(\mathbf{r}) r^{2} d V=\phi(\mathbf{0}) Z e+\frac{Z e}{6}\left\langle r^{2}\right\rangle \nabla^{2} \phi,
$$

with $\left\langle r^{2}\right\rangle$ being the average nuclear radius squared and $\nabla^{2} \phi$ the trace of the EFG tensor. In Mössbauer spectroscopy, the transition between the nuclear ground state $(\mathrm{g})$ and the excited state (e) with differing nuclear radii is measured. Besides, the chemical environments (and consequently $\nabla^{2} \phi$ ) of the source (S) and the absorber (A) are generally different. Using the Poisson equation, $\nabla^{2} \phi$ can be related to the electronic charge at the nucleus, which in turn, is proportional to the square of the total electron wave function $\Psi(\mathbf{r})$. Therefore the isomer shift $\delta$ can be written as:

$$
\delta=\frac{Z e^{2}}{6 \epsilon_{0}}\left(\left\langle r_{g}^{2}\right\rangle-\left\langle r_{e}^{2}\right\rangle\right)\left(\left|\Psi(\mathbf{0})_{S}\right|^{2}-\left|\Psi(\mathbf{0})_{A}\right|^{2}\right) .
$$

The last integral in the r.h.s. of Eq. (4.33) determines the electric quadrupole interaction. The quantity:

$$
e Q=\int \rho(\mathbf{r})\left(3 x_{\alpha}^{2}-r^{2}\right) d V
$$

is a traceless tensor that can be related to the nuclear spin states $(I, \widehat{\mathbf{I}})$ using the WignerEckart theorem [83], and the quadrupole energy becomes:

$$
\widehat{\mathbf{W}}_{Q S}=\sum_{\alpha=1}^{3}\left[\phi_{\alpha \alpha} \frac{e Q}{2 I(2 I-1)}\left(\widehat{\mathbf{I}}_{\alpha}^{2}-\frac{I(I+1)}{3}\right)\right] .
$$

It can be demonstrated that the previous term depends only on the traceless part $V_{\alpha \alpha}=\phi_{\alpha \alpha}-\frac{\nabla^{2} \phi}{3}$ of the electric field gradient tensor [77]. With a proper choice of the coordination system, we can always have:

$$
\left|V_{z z}\right| \geq\left|V_{x x}\right| \geq\left|V_{y y}\right| .
$$


Defining the asymmetry parameter as:

$$
\eta=\frac{V_{x x}-V_{y y}}{V_{z z}}
$$

Eq. (4.37) becomes [79]:

$$
\widehat{\mathbf{W}}_{Q S}=\frac{e Q V_{z z}}{4 I(2 I-1)}\left[3 \widehat{\mathbf{I}}_{z}^{2}+\frac{\eta}{2}\left(\widehat{\mathbf{I}}_{+}^{2}+\widehat{\mathbf{I}}_{-}^{2}\right)-I(I+1)\right]
$$

where $\widehat{\mathbf{I}}_{+}$and $\widehat{\mathbf{I}}_{-}$are the rising and the lowering operators, respectively. For ${ }^{57} \mathrm{Fe}$, the spins of the ground and the excited states are $1 / 2$ and $3 / 2$, respectively, and only the excited states splits into a doublets with separation $\varepsilon$ given by:

$$
\varepsilon=\frac{e Q V_{z z}}{2} \sqrt{1+\frac{\eta^{2}}{3}} .
$$

In the presence of a magnetic field $B$ at the nucleus, the degeneracy of the nuclear states is broken by the interaction energy $-\mu \cdot \mathbf{B}$, where $\mu$ is the nuclear magnetic moment. The state with spin $I$ splits into $2 I+1$ levels with eigenvalues:

$$
E_{m}=-g_{N} \mu_{N} m_{I} B
$$

with $g_{N}$ being the Landé factor, $\mu_{N}$ the nuclear Bohr magneton and $m_{I}=I, I-1, \ldots,-(I-$ $1),-I$ the magnetic quantum number. There are 4 level for the excited state and 2 for the ground state of ${ }^{57} \mathrm{Fe}$, for a total of 8 transitions, but the selection rules $\Delta m_{I}=0, \pm 1$ allow only six of them, leading to the characteristic ${ }^{57} \mathrm{Fe}$ Mössbauer sextet of magnetic materials, as shown in Fig. 4.9. The intensity of each transition line depends on the

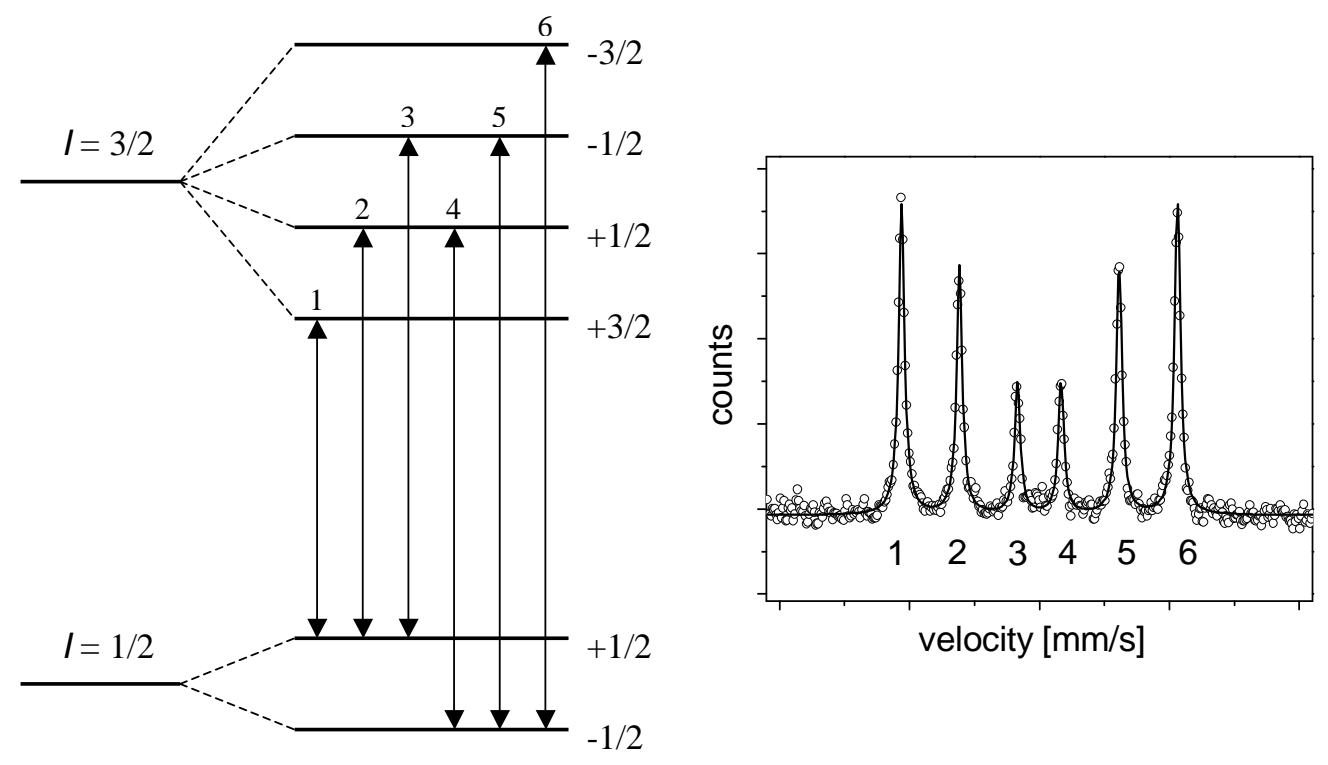

Figure 4.9: Sketch of the ${ }^{57}$ Fe Mössbauer transitions scheme (left) and typical $\alpha$-Fe Mössbauer spectrum (right). All the corresponding transition lines are labeled.

angle $\theta_{m}$ between the direction of propagation of the $\gamma$-rays and the hyperfine magnetic field as summarized in Table 4.3. The standard fitting routine of the Mössbauer spectra 
Table 4.3: Angular dependence of the Mössbauer transition lines.

\begin{tabular}{ccc}
\hline \hline Transition & $\Delta m_{I}$ & Angular dependence \\
\hline $\pm 3 / 2 \rightarrow \pm 1 / 2$ & \pm 1 & $3 / 4\left(1+\cos ^{2} \theta_{m}\right)$ \\
$\pm 1 / 2 \rightarrow \pm 1 / 2$ & 0 & $\sin ^{2} \theta_{m}$ \\
$\mp 1 / 2 \rightarrow \pm 1 / 2$ & $\mp 1$ & $1 / 4\left(1+\cos ^{2} \theta_{m}\right)$ \\
\hline \hline
\end{tabular}

is performed using superimposed lorentzian lines, and the fitting parameters are: the line width $\Gamma$ (Eq. (4.29)), the isomer shift $\delta$ (Eq. (4.35)), the quadrupole splitting $\varepsilon$ (Eq. (4.41)), the magnetic hyperfine field $B$ (Eq. 4.42) $)$ and the relative intensities of the lines (Table 4.3). The typical geometries of a Mössbauer experiment are sketched in Fig. 4.10, The radioactive ${ }^{57} \mathrm{Co}(\mathrm{Rh})$ source $\left({ }^{57} \mathrm{Co}\right.$ in $\mathrm{Rh}$ matrix, typical activity of



Figure 4.10: Geometries of the transmission (TMS) and the conversion (CEMS-CXMS) Mössbauer experiments.

$10 \mathrm{mCi}$ ) is mounted on a computer-controlled drive, moving periodically toward and away from the sample with a frequency of the order of $10 \mathrm{~Hz}$. In transmission geometry (TMS) the $\gamma$-ray detector is placed behind the sample. The $\gamma$-ray yield is recorded in a multichannel analyzer as a function of the source velocity (i.e. the energy modulation). When the velocity is such that the resonant energy is reached, a $\gamma$-ray can be absorbed by the sample, and then re-emitted in random direction. Therefore, at the resonant energy, the detector will count fewer photons and the spectral lines appear downward. In backscattering geometry the sample is placed on the bottom of the detector and the conversion phenomenon is used [84]. The photon emitted from an excited nucleus might interact with the inner shell electrons leading to the emission of electrons and X-rays instead of $\gamma$-rays. If the detector reveals electron, the technique is named Conversion Electron Mössbauer Spectroscopy (CEMS). When the X-rays are counted, we have the Conversion X-ray Mössbauer Spectroscopy (CXMS). In both cases, more electrons and 
X-rays are revealed at the resonant energy and the spectral lines are upward. It should be mentioned that the information depth of CEMS is about $150 \mathrm{~nm}$, while CXMS can reach $10 \mu \mathrm{m}$ [85].

\subsection{X-Ray Absorption Fine Structure}

X-ray absorption fine structure (XAFS) refers to the oscillatory structure of the linear X-ray absorption coefficient $\mu$ above the absorption edge [86]. The intensity $I_{0}$ of a Xray beam traversing a solid sample decreases exponentially with the penetration depth according to the following law:

$$
I(z)=I_{0} \exp (-\mu z)
$$

where $\mu$ depends on the nature of the target and in general on the X-ray energy. The absorption coefficient sharply increases when the X-ray energy matches the energy $E_{c}$ of a inner electronic shell: the abrupt jump of $\mu(E)$ at the energy $E=E_{c}$ corresponds to the photoexcitation of an electron from the atomic core level with energy $E_{c}$. Above the edge $E_{c}$ some weak wiggles of $\mu(E)$ are observed. These oscillations are characteristic of the emitting sample and they provide a unique signature of the atomic structure of the material. They are interpreted as a quantum interference phenomenon: the outgoing electron of momentum $p=\hbar k$ can be viewed as a quantum oscillation of wavelength $\lambda=h / p=2 \pi / k$ propagating over the solid. This waves can be scattered by the atoms surrounding the emitter, and the amplitude of the reflected waves can add constructively or destructively to the original outgoing wave. The interference pattern changes with the energy of the photoelectron (i.e. with $k$ ) and consequently the absorption coefficient will exhibit similar oscillations. A XAFS spectrum consists on the measurement of the X-ray absorption coefficient $\mu(E)$ as a function of the X-ray energy, more specifically the intensities $I_{0}$ and $I\left(z^{\prime}\right)$ of the incident and the transmitted X-ray beams, respectively, are measured. For a sample of given thickness $z^{\prime}$ we have:

$$
\mu=\frac{1}{z^{\prime}} \ln \left[\frac{I_{0}}{I\left(z^{\prime}\right)}\right]
$$

The XAFS spectrum $\chi(E)$ is defined as the normalized, oscillatory part of the absorption coefficient above the edge [87]:

$$
\chi(E)=\left[\mu(E)-\mu_{0}(E)\right] / \Delta \mu_{0}
$$

where $\mu_{0}(E)$ is the smooth, atomic-like component of the absorption coefficient and $\Delta \mu_{0}$ is a normalization factor (typically the magnitude of the jump at the absorption edge). An example is shown in Fig. 4.11, where the measured absorption coefficient $\mu$, its smooth atomic-like component $\mu_{0}$ and the extrapolated $\chi$ of a crystalline Si sample are reported. Each absorption edge is related to a specific atom and the K-edge is considered in most XAFS experiments. The energies of the K-edge for same elements of interest in this work are reported in Table 4.4. The weak oscillatory structure $30 \mathrm{eV}$ above the edge is generally termed EXAFS (Extended X-ray Absorption Fine Structure) and it contains the information about the local atomic surrounding of the atoms that absorbed the X-ray. In 




Figure 4.11: X-ray absorption coefficient $\mu$ (solid line), the smooth atomic-like component $\mu_{0}$ (dashed line) and the extrapolated $\chi$ (bold solid line) of a crystalline Si sample.

Table 4.4: K-edge energies of carbon, nitrogen, aluminum, silicon and iron.

\begin{tabular}{lcc}
\hline \hline Element & Atomic number & K-edge energy $[\mathrm{eV}]$ \\
\hline Carbon & 6 & 284.2 \\
Nitrogen & 7 & 409.9 \\
Aluminum & 13 & 1559 \\
Silicon & 14 & 1839 \\
Iron & 56 & 7112 \\
\hline \hline
\end{tabular}

contrast, the region closer to the edge (within the first $30 \mathrm{eV}$ ) is known as XANES (X-ray Absorption Near-Edge Structure) and is dominated by the strong multiple scatterings and by the atomic resonances [86]. The experimental data are typically collected in the energy region that goes from a few tens of $\mathrm{eV}$ below the edge to several hundreds of $\mathrm{eV}$ above the edge in energy step of constant width (e.g. $1 \mathrm{eV})$. If the X-ray photon has an energy $\hbar \omega>E_{c}$, the extra energy is transformed into kinetic energy of the emitted photoelectron. Conventionally $\chi$ is defined with respect to the photoelectron momentum $k=\sqrt{2 m\left(\hbar \omega-E_{c}\right) / \hbar^{2}}$ obtaining $\chi(k)$ instead of $\chi(E)$. The parametrization of the XAFS pattern was formalized by Sayers et al. [88] in 1971 and it can be summarized in the following form:

$$
\chi(k)=\sum_{i} A(k)_{i} N_{i} \frac{\sin \left[2 k R_{i}+\delta_{i}(k)\right]}{R_{i}^{2}} \exp \left(-2 R_{i} / \eta\right) \exp \left(-2 k^{2} \sigma_{i}^{2}\right) .
$$

The index $i$ refers to the atomic shell, and the sum is performed over all shells: $N_{i}$ are the coordination numbers (i.e. the number of atoms in each shell), $R_{i}$ are the interatomic 
distances (i.e. the radii of the shells), $A_{i}(K)$ are the scattering amplitudes, $\delta_{i}(k)$ are phase shifts, $\eta$ is the XAFS mean free path and $\sigma_{i}$ are the average fluctuations of the interatomic bond lengths. From Eq. (4.46) it is clear that the XAFS pattern is given by the superposition of different coordination shells, each oscillating with different amplitude and frequency. In order to analyze the data, it is necessary to isolate each contribution. The standard method is to perform the Fourier Transform (FT) of the pattern. The atomic like part $\mu_{0}$ of the absorption coefficient is extrapolated from the experimental data using cubic splines or a polynomial fit. Once the $\chi(E)$ is obtained, it is converted to $\chi(k)$ and then Fourier tranformed. Due to the finite data range, the FT can be contaminated by spurious peaks or oscillations. Therefore, the $\chi(k)$ is usually convoluted with a window $W(k)$ that smoothly goes to zero at the edges of the $k$-range. Besides, the data are weighted with some power of $k\left(k^{n}\right.$, with $n=1,2$ or 3$)$ to compensate the decay of the waves at large values of $k$. The FT is thus performed as follows [87]:

$$
\tilde{\chi}(R)=\int_{k_{\min }}^{k_{\max }} e^{-i k r} W(k) k^{n} \chi(k) d k
$$

Each peak of $\tilde{\chi}(R)$ represents a shell contribution. The position of the peaks is related (but not equal) to the radius of the shells, and the intensity of the peaks reflects the number of atoms in the shell. The quantitative analysis is performed selecting one or few adjacent

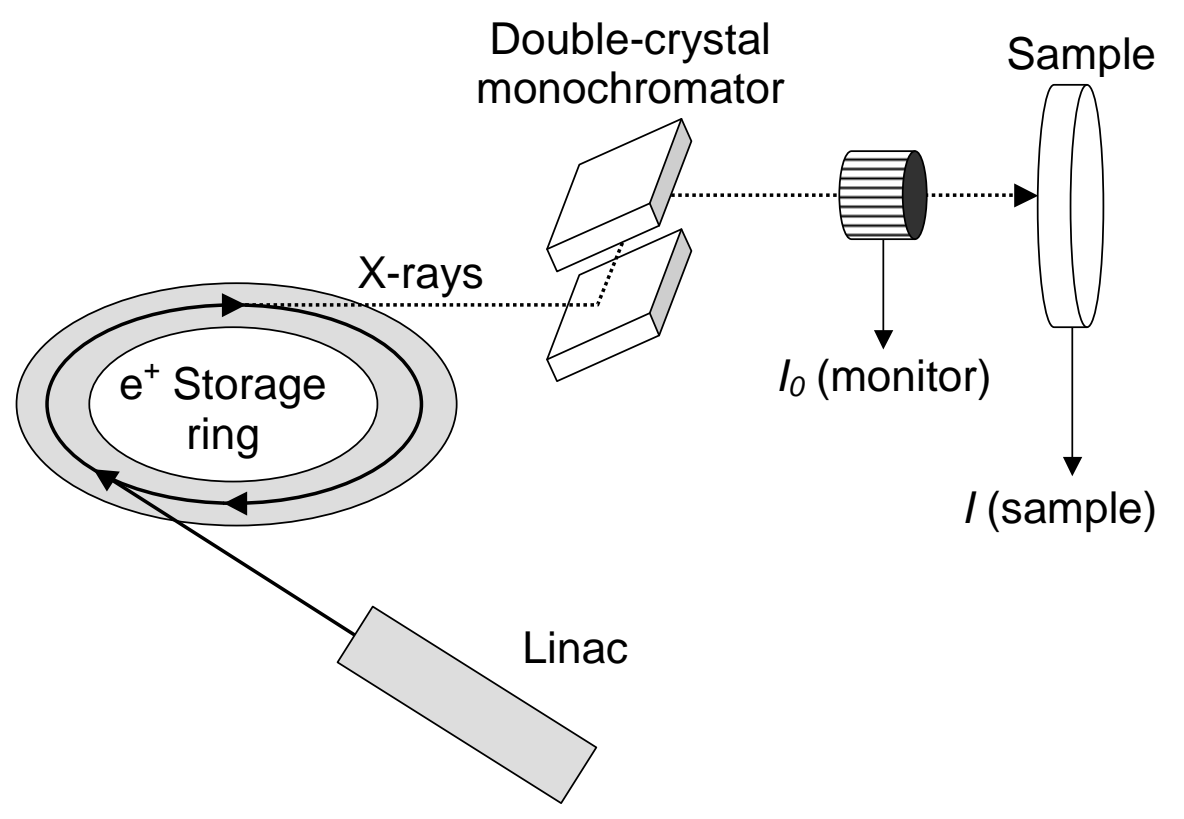

Figure 4.12: Sketch of the L.U.R.E. SA32 beamline experimental setup.

peaks and calculating the back Fourier transform (BFT). The corresponding oscillations in the $k$-space are then fitted according to Eq. (4.46), in order to obtain the values of the coordination number $N_{i}$, the interatomic distance $R_{i}$ and the disorder parameter $\sigma_{i}$ of each shell. The scattering amplitudes $A_{i}$ and the phase shifts $\delta_{i}$ are entered as initial parameters. They are calculated with the help of dedicated softwares [89] or obtained experimentally from the analysis of calibration samples. Similarly to Mössbauer spectroscopy, the XAFS data can be collected in transmission geometry, where the incident and the transmitted X-ray intensities are compared, or in backscattering geometry where the emitted photoelectrons are detected. The latter is called total electron yield (TEY) 
mode. The XAFS technique has been employed to analyze the silicon substrates irradiated in nitrogen and in methane atmospheres. All data have been collected at the L.U.R.E (Laboratoire pour l'Utilisation du Rayonnement Electromagnétique, Orsay - France) on the beamline SA32. The experimental setup is schematically drawn in Fig. 4.12. The SuperACO storage ring was operating with a positron beam at $800 \mathrm{MeV}$ and average current of $200 \mathrm{~mA}$. The X-ray energy was selected with an InSb $<111>$ double-crystal monochromator and the data were collected at room temperature in the energy range 1800-2500 eV, with $1 \mathrm{eV}$ step in TEY mode. The investigated depth was estimated to be about $30 \mathrm{~nm}$ according to the formula given by Elam et al. [90]. The data analysis was performed with the VIPER software [91, 92] using weighted cubic spline functions to simulate the continuous, atomic-like $\mu_{0}$. The FT was performed in the region $2 \AA^{-1}<k<12 \AA^{-1}$ using the $k^{2}$ weight and a gaussian window $W(k)$ centered at $\bar{k}=7.0 \AA^{-1}$ and defined as follows: $W(k)=\exp \left[-3 \pi(k-\bar{k})^{2} / 2 \bar{k}^{2}\right]$.

\subsection{Crystallography and X-Ray Diffraction}

The X-ray Diffraction (XRD) is used for the structural analysis of crystalline samples. Since the typical interatomic distances in a solid are of the order of $10^{-10} \mathrm{~m}$, an electromagnetic probe of the microscopic structure must have a wavelength $\lambda$ this short, corresponding to an energy $h c / \lambda=h c / 10^{-10} \mathrm{~m} \simeq 10^{4} \mathrm{eV}$. These are the characteristic $\mathrm{X}$-ray energies. The scattering process by a perfect periodic structure can be explained in two equivalent ways, due to Bragg and to von Laue [53. Referring to Fig. 4.13, the Bragg formulation assumes the crystal as made of parallel planes of ions called lattice planes, placed a distance $d$ apart. The conditions to have a sharp, intense peak of the scattered

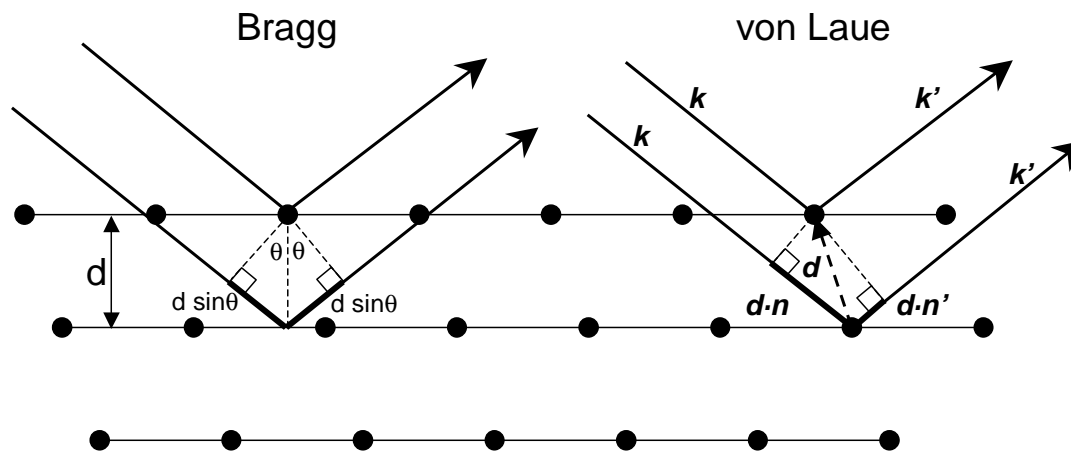

Figure 4.13: Bragg (left) and von Laue (right) pictorial explanations of the X-ray diffraction phenomenon.

radiation are: (i) the X-ray should be specularly reflected and (ii) the reflected waves should interfere constructively. The difference in the optical path of two X-ray scattered from successive planes is simply $2 d \sin \theta$ where $\theta$ is the incident angle. To have constructive interference, this path must equal an integer number $n$ of wavelengths, leading to the celebrated Bragg condition:

$$
2 d \sin \theta=n \lambda .
$$

The alternative way to explain the X-ray diffraction is due to von Laue, who considered the crystal as given by identical microscopic objects (atoms) regularly placed. If two 
scatterers are separated by a displacement vector $\mathbf{d}$, the incoming X-ray of wavelength $\lambda$ along the direction $\widehat{\mathbf{n}}$ can be described by the wave vector $\mathbf{k}=2 \pi \widehat{\mathbf{n}} / \lambda$. Similarly, the scattered X-ray in a direction $\widehat{\mathbf{n}}^{\prime}$ is described by the wave vector $\mathbf{k}^{\prime}=2 \pi \widehat{\mathbf{n}}^{\prime} / \lambda$. The difference in the optical paths is simply $\mathbf{d} \cdot\left(\widehat{\mathbf{n}}-\widehat{\mathbf{n}}^{\prime}\right)$ and the condition of constructive interference becomes:

$$
\mathbf{d} \cdot\left(\widehat{\mathbf{n}}-\widehat{\mathbf{n}}^{\prime}\right)=n \lambda
$$

which, in terms of wave vectors, is:

$$
\mathbf{d} \cdot\left(\mathbf{k}-\mathbf{k}^{\prime}\right)=2 \pi n \text {. }
$$

or also:

$$
e^{i \mathbf{d} \cdot\left(\mathbf{k}-\mathbf{k}^{\prime}\right)}=1 .
$$

It can be demonstrated that Eq. (4.48) and Eq. (4.51) are equivalent (see for example [53], chapter 6). The basic concept to describe a crystalline solid is the Bravais lattice, that specifies the periodicity of the atomic arrangement. It consists of all points with positions $\mathbf{R}$ of the form:

$$
\mathbf{R}=m_{1} \mathbf{v}_{\mathbf{1}}+m_{2} \mathbf{v}_{\mathbf{2}}+m_{3} \mathbf{v}_{\mathbf{3}}
$$

where $\mathbf{v}_{\mathbf{i}}(i=1,2,3)$ are called primitive vectors and $m_{i}$ are integer numbers. The primitive cell is the portion of space that, translated through all the primitive vectors, fills the space without overlaps or voids. A physical crystal is completely described by giving its Bravais lattice and the arrangement of molecules/atoms/ions inside the primitive cell. The structure of such crystal consists of identical copies of the same unit, called basis, located at the points of the Bravais lattice. An example is reported in Fig. 4.14. For a

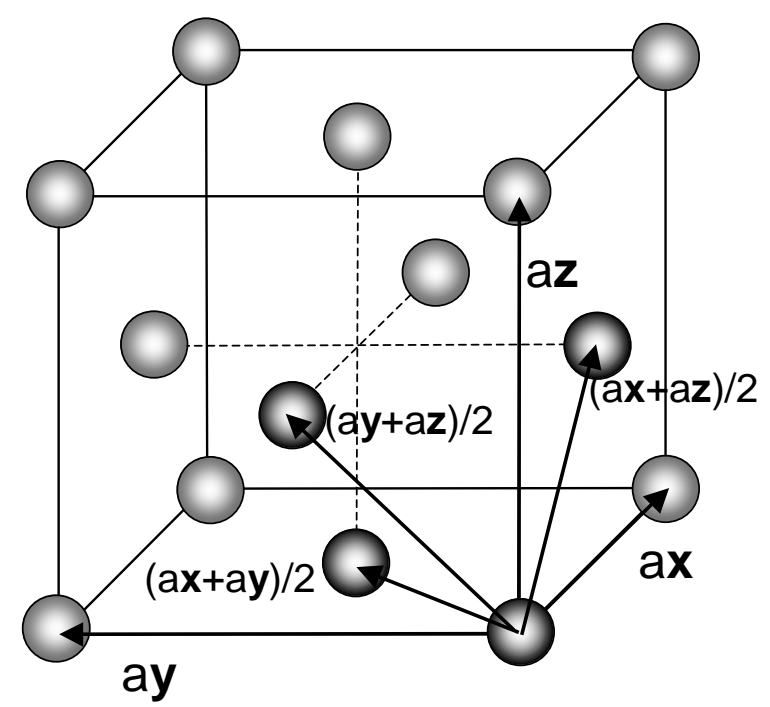

Figure 4.14: Face centered cubic (fcc) Bravais lattice system with lattice parameter $a$. The four darker atoms constitute the basis.

given Bravais lattice $\mathbf{R}$, the reciprocal lattice is defined as the set of vectors $\mathbf{K}$ that gives plane waves with the periodicity of the given Bravais lattice. Analytically:

$$
e^{i \mathbf{K} \cdot \mathbf{R}}=1
$$


As an example, for a cubic crystal of lattice parameter $a, \quad \mathbf{R}=a\left(m_{1} \widehat{\mathbf{x}}+m_{2} \widehat{\mathbf{y}}+m_{3} \widehat{\mathbf{z}}\right)$ and the reciprocal lattice vectors satisfying Eq. 4.53$)$ are $\mathbf{K}=2 \pi / a\left(k_{1} \widehat{\mathbf{x}}+k_{2} \widehat{\mathbf{y}}+k_{3} \widehat{\mathbf{z}}\right)$, being $m_{1}, m_{2}, m_{3}, k_{1}, k_{2}$ and $k_{3}$ integer numbers. The reciprocal lattice plays an important role in crystallography, since it identifies all families of crystallographic planes: a lattice plane is defined as any plane containing at least three non-collinear points of the Bravais lattice, and a family of lattice planes is the set of parallel, equally spaced lattice planes that together contain all the points of the Bravais lattice. For any family of lattice planes a distant $d$ apart, there are reciprocal lattice vectors, the shortest of which has length $2 \pi / d$ [53]. The Miller indices (usually $h, k, l$ ) of a lattice plane are the coordinates of the shortest reciprocal lattice vector normal to that plane. Comparing Eq. (4.51) with Eq. 4.53) it is clear that the change of the wave vector $\mathbf{K}=\mathbf{k}-\mathbf{k}^{\prime}$ must belong to the reciprocal lattice, in order to have constructive interference. For a cubic crystal of lattice parameter $a, \quad \mathbf{K}=2 \pi / a(h \widehat{\mathbf{x}}+k \widehat{\mathbf{y}}+l \widehat{\mathbf{z}})$. By definition, the family of lattice planes $\{h k l\}$ must satisfy the condition

$$
|\mathbf{K}|^{2}=\left(\frac{2 \pi}{a}\right)^{2}\left(h^{2}+k^{2}+l^{2}\right)=\left(\frac{2 \pi}{d}\right)^{2}
$$

and using the Bragg's law (Eq. (4.48) with $n=1$ ) to eliminate $d$, we obtain

$$
\lambda^{2} \frac{\left(h^{2}+k^{2}+l^{2}\right)}{4 a^{2}}=\sin ^{2} \theta .
$$

Each family of lattice planes $\{h k l\}$ corresponds to a Bragg peak in the angular position $\theta$, or equivalently with scattering angle $2 \theta$. In nature there are 7 crystal systems and 14 Bravais lattices, as summarized in Table 4.5. Relations like Eq. 4.55) can be found

\begin{tabular}{|c|c|c|c|}
\hline Crystal system & Bravais lattice & Lattice constants & Lattice angles \\
\hline Cubic & $\begin{array}{l}\text { simple } \\
\text { body-centered } \\
\text { face-centered }\end{array}$ & $a=b=c$ & $\alpha=\beta=\gamma=90^{\circ}$ \\
\hline Tetragonal & $\begin{array}{l}\text { simple } \\
\text { centered }\end{array}$ & $a=b \neq c$ & $\alpha=\beta=\gamma=90^{\circ}$ \\
\hline Orthorhombic & $\begin{array}{l}\text { simple } \\
\text { base-centered } \\
\text { body-centered } \\
\text { face-centered }\end{array}$ & $a \neq b \neq c$ & $\alpha=\beta=\gamma=90^{\circ}$ \\
\hline Monoclinic & $\begin{array}{l}\text { simple } \\
\text { centered }\end{array}$ & $a \neq b \neq c$ & $\beta \neq \alpha=\gamma=90^{\circ}$ \\
\hline Triclinic & simple & $a \neq b \neq c$ & $\alpha, \beta, \gamma \neq 90^{\circ}$ \\
\hline Trigonal & simple & $a=b=c$ & $\alpha=\beta=\gamma \neq 90^{\circ}$ \\
\hline Hexagonal & simple & $a=b, c$ & $\alpha=\beta=90^{\circ}, \gamma=120^{\circ}$ \\
\hline
\end{tabular}

Table 4.5: Crystal systems and Bravais lattices.

for each of them, revealing that the the diffraction directions (i.e. the diffraction angles) are determined only by the shape and the size of the unit cell. On the other hand, the 
intensity of each diffraction peak is affected by the arrangement of atoms inside the unit cell and by the geometry of the XRD experiment. The structure factor $F_{h k l}$ is in general a complex number describing the amplitude and the phase of the wave scattered by all $N$ atoms in the unit cell [93]:

$$
F_{h k l}=\sum_{n=1}^{N} f_{n} e^{2 \pi i\left(h u_{n}+k v_{n}+l w_{n}\right)},
$$

where $u_{n}, v_{n}$ and $w_{n}$ are the fractional coordinates of the $n^{\text {th }}$ atom inside the unit cell, and $f_{n}$ is the corresponding scattering factor. The Lorentz-polarization factor $\phi_{L P}$ depends on the geometry of the XRD experiment and on the polarization of the X-ray beam (typically randomly polarized) [93]:

$$
\phi_{L P}=\frac{1+\cos ^{2} 2 \theta}{\sin ^{2} \theta \cos \theta} .
$$

The temperature factor produces a decrease in the intensity of the diffracted peaks. It depends on the thermal vibrations that atoms undergo about their equilibrium positions, and is quantified as $e^{-2 M}$ with [93]:

$$
M=\frac{6 h^{2}}{m k_{B} \Theta}\left[\frac{1}{4}+\frac{T^{2}}{\Theta^{2}} \int_{0}^{\Theta / T} \frac{x d x}{e^{x}-1}\right]\left(\frac{\sin \theta}{\lambda}\right)^{2} .
$$

The angular dependence of the Lorentz-polarization $\times$ temperature factors is shown in Fig. 4.15 for pure Fe and pure $\mathrm{Al}$ specimens. The multiplicity factor $p$ is the number of

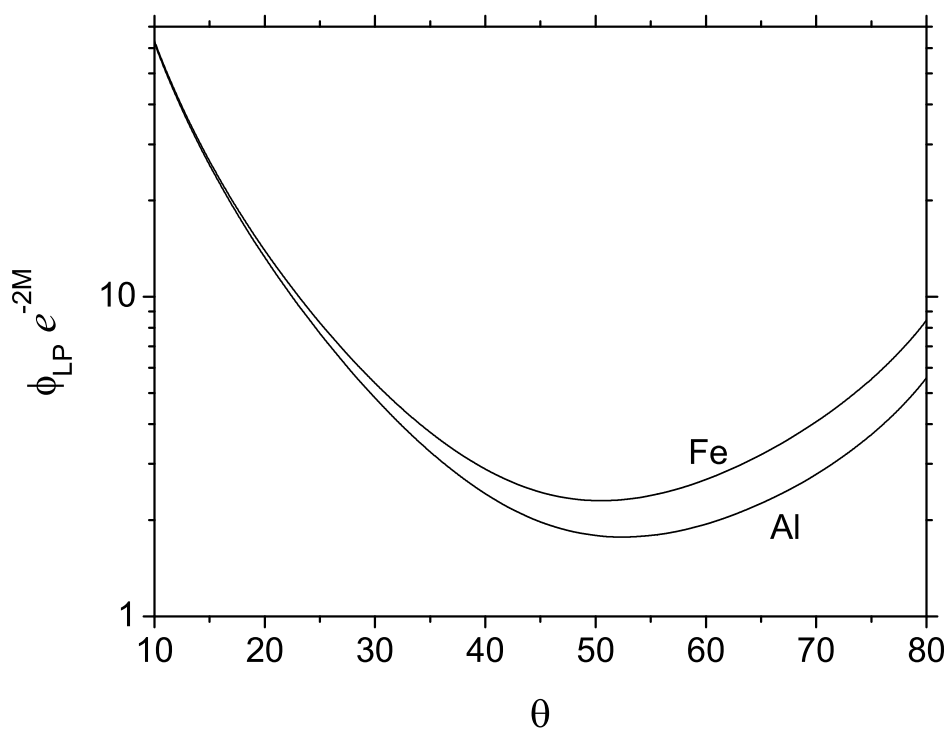

Figure 4.15: Angular dependence of the Lorentz-polarization $\times$ temperature factors for $\mathrm{Fe}$ and Al substrates.

equivalent directions contributing to the same reflection (for example, in a cubic crystal the [110], [101] and [011] directions are all equivalent and indicated as $<110>$ ). Therefore, the intensity $I_{h k l}$ of a given diffraction peak can be written as:

$$
I_{h k l}=\left|F_{h k l}\right|^{2} p\left(\frac{1+\cos ^{2} 2 \theta}{\sin ^{2} \theta \cos \theta}\right) e^{-2 M} .
$$


All the diffractograms reported in this work have been recorded with a Bruker AXS diffractometer employing a $\mathrm{Cu}$ source $(\lambda=1.54 \AA)$ and $\mathrm{LiF}$ single-crystal monochromator on the diffracted beam. Most of the data have been collected in glancing incidence geometry (GIXRD), the basic principle of which is sketched in Fig. 4.16. The incidence angle $\alpha$

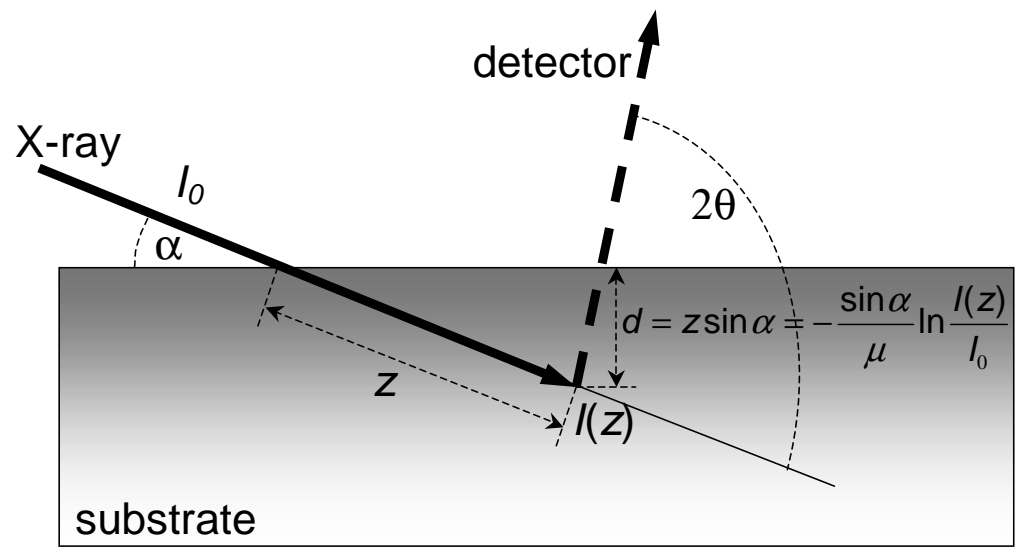

Figure 4.16: Geometry of the GIXRD experiment. The intensity $I_{0}$ of the incident X-rays is reduced to $I(z)$ after traveling a distance $z$ inside the substrate, corresponding to a depth $d=z \sin \alpha$.

between the incoming X-rays and the substrate surface is fixed, while the angle between the incoming $\mathrm{X}$-rays and the rotating detector is $2 \theta$. With simple geometrical considerations and using Eq. (4.43), the X-ray penetration depth $d$ can be calculated at any given incidence angle for any material (the linear absorption coefficients $\mu$ of the $\mathrm{Cu}-\mathrm{K} \alpha$ radiation are tabulated in 93 . for all the elements of the periodic table). Table 4.6 reports the penetration depths $[\mu \mathrm{m}]$ at which $90 \%$ of the X-ray intensity has been absorbed (i.e. $I(z) / I_{0}=0.1$, see Fig. 4.16), for three incidence angles $\left(1^{\circ}, 3^{\circ}\right.$ and $\left.5^{\circ}\right)$ in pure Fe, pure $\mathrm{Al}$ and pure Si substrates.

Table 4.6: Penetration depth $[\mu \mathrm{m}]$ at which $90 \%$ of the $\mathrm{Cu}-\mathrm{K} \alpha$ X-ray intensity is absorbed in $\mathrm{Fe}, \mathrm{Al}$ and $\mathrm{Si}$ targets at incidence angles of $1^{\circ}, 3^{\circ}$ and $5^{\circ}$.

\begin{tabular}{lccc}
\hline \hline Element & \multicolumn{3}{c}{ glancing angle [ $\left.{ }^{\circ}\right]$} \\
\cline { 2 - 4 } & 1 & 3 & 5 \\
\hline $\mathrm{Fe}$ & 0.17 & 0.50 & 0.84 \\
$\mathrm{Al}$ & 1.29 & 3.86 & 6.43 \\
$\mathrm{Si}$ & 1.15 & 3.44 & 5.73 \\
\hline \hline
\end{tabular}

Some detailed analysis required the use of the Rietveld refinement [94, 95], that was carried out with the FULLPROF software package [96] (for a review of the Rietveld method see [97]). 


\subsection{Nanoindentation hardness}

The hardness of a material can be defined as its resistance to the penetration of a harder tip [43]. The current method to measure the hardness is to employ a diamond tip as the

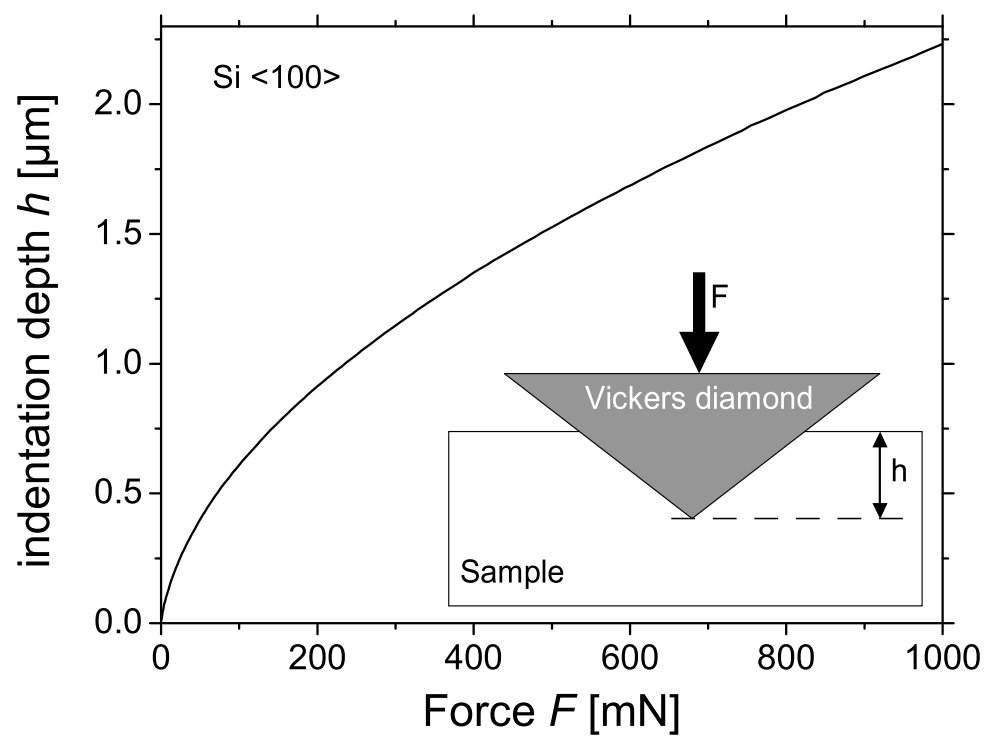

Figure 4.17: Indentation depth vs. load applied on a Vickers diamond tip indenting a single crystalline Si substrate. The inset represents the experimental geometry.

indenter and to determine the indentation depth $h$ as a function of the applied load $F$. The typical $h-F$ curve of a single-crystalline silicon substrate, $<100>$ oriented is shown in Fig. 4.17. The universal hardness $H\left[\mathrm{~N} / \mathrm{mm}^{2}\right.$ or $\left.\mathrm{MPa}\right]$ is defined as the ratio of the applied force $F$ and the area of contact $A$ between the material and the diamond tip, which in turn is function of the indentation depth. For a Vickers diamond indenter, the tip is a square-based pyramid with an opening angle of $136^{\circ}$, and the contact area $A$ is given by $A=4 h^{2} \sin \left(68^{\circ}\right) / \cos ^{2}\left(68^{\circ}\right)=26.43 h^{2}$. Therefore the universal hardness $H$ is:

$$
H=\frac{F}{A}=\frac{F}{26.43 h^{2}}
$$

In reality, the Vickers diamond is never a perfect pyramid especially at its vertex, and any geometrical deviation leads to large errors in the estimation of the hardness. To overcome this problem, the experimental setup is calibrated by indenting a homogeneous material of known hardness (typically $<100>$ single crystalline $\mathrm{Si}$ ): the measured hardness is corrected in order to match its real value, and the correction function is stored in a computer. Each indentation profile is then corrected accordingly. An example of hardness curve before and after the correction is reported in Fig. 4.18. All hardness measurements shown in the next chapters have been performed with a Fisherscope HV 100 allowing a depth resolution of few nanometers (depending on the surface roughness) and a force definition that can be better than $50 \mathrm{nN}$. The maximum load is chosen according to the analyzed material (typically less than $1 \mathrm{~N}$ ); the indentation curve is obtained by increasing 


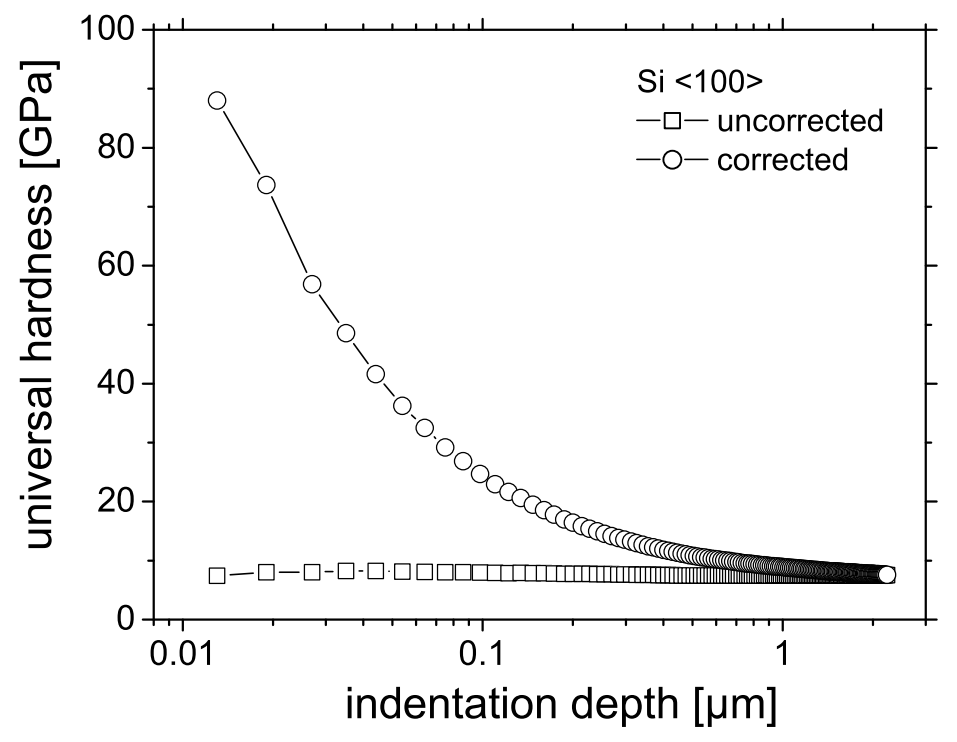

Figure 4.18: Corrected and non-corrected hardness of a single crystalline Si substrate.

the square root of the load with constant increments (150 steps in total) and measuring the corresponding indentation depth at each step. The force is applied for a tenth of a second in each step. The data acquisition procedure is computer-controlled and requires few minutes. In general, 20 to 50 indentation curves are measured at different positions on the surface of the samples, in order to improve the statistics of the acquisition. 


\section{Chapter 5}

\section{The iron substrate}

Laser irradiation of iron in nitrogen atmosphere (iron laser nitriding) has been extensively investigated in the past years, determining the influence of the laser fluence, the number of shots, the pulse duration and wavelength, and the nitrogen gas pressure on the efficiency of the treatment. The results are reported in Ref. [28, 29, 30, 31, 98, 99, 100] and only the most recent findings on the phenomenological modeling of the mass transport mechanism will be discussed here [101]. Due to the successful treatment of iron in nitrogen atmosphere, the laser process has been extended to different kinds of gaseous environments. The results of the laser irradiation in methane $\left(\mathrm{CH}_{4}\right)$ will be reported [102, 103], showing the effectiveness of the carbon incorporation into the iron matrix.

\subsection{The nitrogen transport mechanism}

The following sections will describe a phenomenological model (based on the experimental evidence) of the nitrogen transport mechanism during single spot irradiations of iron in $\mathrm{N}_{2}$ gas. The nitrogen take-up mechanism has already been modeled for the raw laser beam [104]: the nitrogen depth profiles were fitted with a linear combination of two complementary error functions (erfc) and the parametrization vs. the number of laser pulses was obtained. Further experiments have been performed at the laser fluence of $4 \mathrm{~J} / \mathrm{cm}^{2}$ in 1 bar $\mathrm{N}_{2}$ with the homogeneous laser beam, in order to hinder the mass transport due to convection.

\subsubsection{The diffusion process}

Using the capability of RNRA, the contribution of each laser shot on the mass transport mechanism can be distinguished. By irradiating the target in enriched isotopic atmosphere, it is possible to track the evolution of the nitrogen profiles under the effect of subsequent laser pulses. An irradiation in ${ }^{15} \mathrm{~N}$ atmosphere will be marked by a star $\left(^{*}\right)$ and the notation $m^{*} / n$ will indicate $m$ pulses in enriched atmosphere followed by $n$ pulses in natural nitrogen. Fig. 5.1 shows the results of the isotopic experiments $1^{*} / 0,1^{*} / 1$, $1^{*} / 2,1^{*} / 4$, illustrating the evolution of the nitrogen profile produced by the first pulse 
$\left(1^{*} / 0\right)$ after $1\left(1^{*} / 1\right), 2\left(1^{*} / 2\right)$ and $4\left(1^{*} / 4\right)$ subsequent laser shots in natural nitrogen. The first pulse $\left(1^{*} / 0\right)$ can be fitted by a complementary error function (erfc):

$$
e\left(z, t_{e}\right)=C \operatorname{erfc}\left[z / \sqrt{4 D t_{e}}\right]
$$

where $C$ is the surface concentration, $D$ the diffusion constant and $t_{e}$ the diffusion time. The other isotopic experiments $\left(1^{*} / 1,1^{*} / 2,1^{*} / 4\right)$ can be fitted by gaussian profiles:

$$
g_{n}\left(z, t_{g}\right)=\frac{M}{\sqrt{\pi} L_{n} / 2} \exp \left[-z^{2} / L_{n}^{2}\right] \text {. }
$$

$M$ is the amount of ${ }^{15} \mathrm{~N}$ remaining after further irradiations and $L_{n}$ is the diffusion length of the profile after $n$ pulses. As illustrated in Fig. 5.2, the square of $L_{n}$ depends linearly

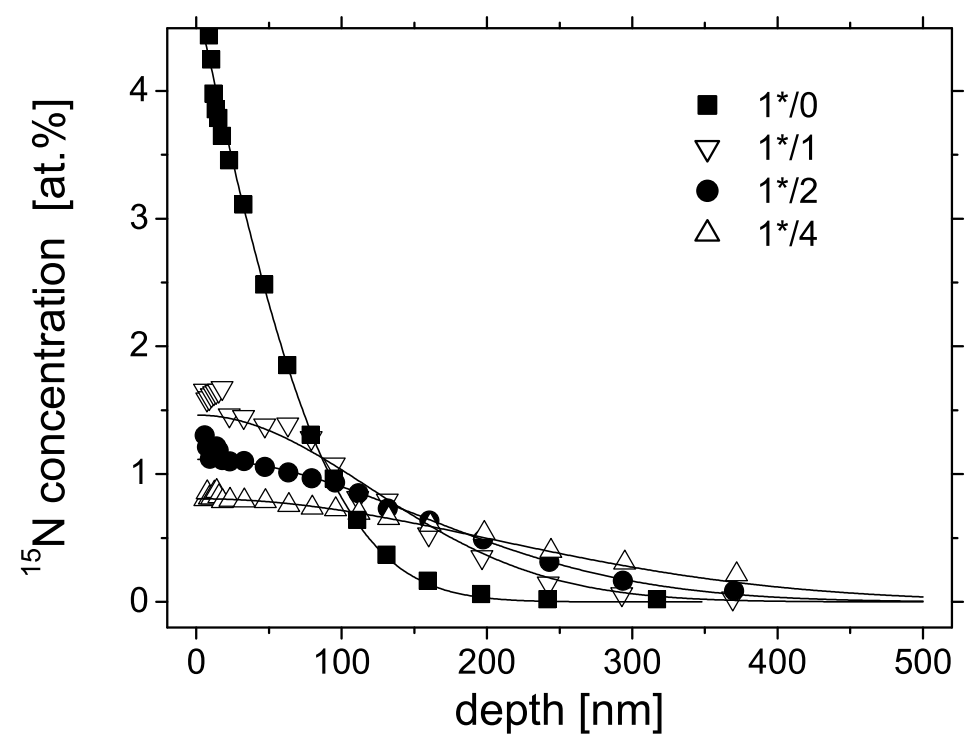

Figure 5.1: Isotopic experiments $1^{*} / 0,1^{*} / 1,1^{*} / 2,1^{*} / 4$ : the symbols represent the experimental data, the solid lines are the simulations.

on $n$ :

$$
L_{n}^{2}=4\left(D t_{e}+n D t_{g}\right) .
$$

$D t_{e}$ is the contribution of the first pulse (the erfc) to the total diffusion length $L_{n}$ and $t_{e}+n t_{g}$ is the total diffusion time of the profile after $n+1$ pulses. Eq. (5.3) is a clear evidence of the diffusion nature of the process: the increase in the diffusion length is due to the linear increase in the total diffusion time with the number of laser pulses. It should be noticed that both Eq. (5.1) and Eq. (5.2) are solutions of the one-dimensional diffusion equation:

$$
\frac{\partial C(z, t)}{\partial t}=D \frac{\partial^{2} C(z, t)}{\partial z^{2}},
$$

where $C(z, t)$ is the solute concentration and the diffusion constant $D$ is assumed to be independent of the concentration itself, the time $t$, the position $z$ and the substrate 


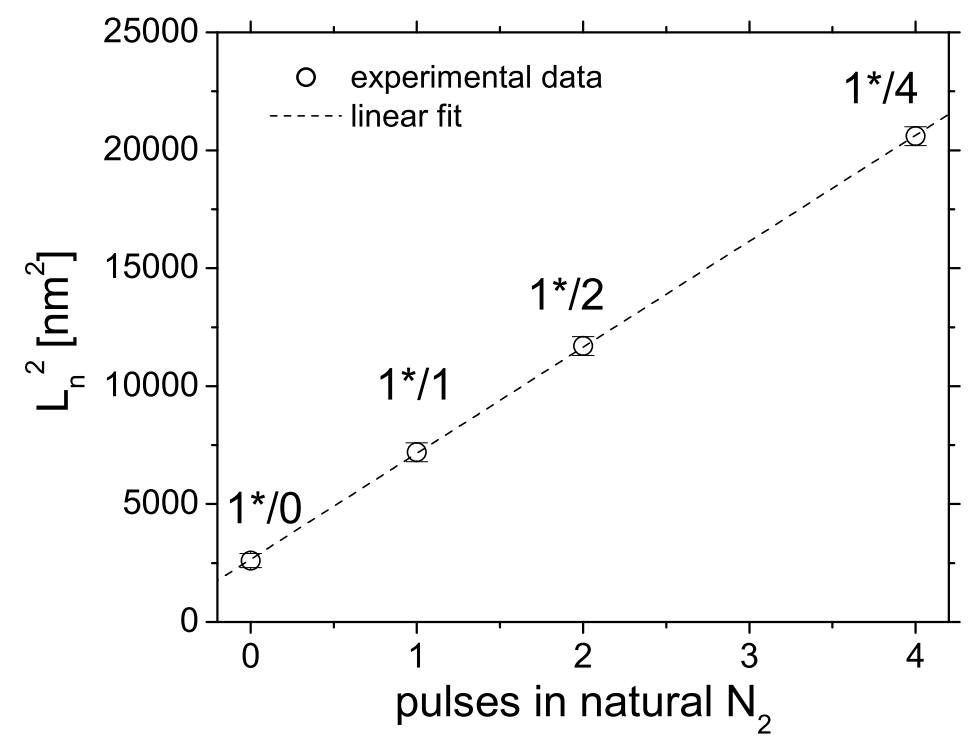

Figure 5.2: Diffusion length squared of the depth profiles as a function of the number of pulses.

temperature (the use of one-dimensional equations was justified in section 3.1). The experimental value of $C$ is 4.1 at.\%, while $D t_{e}$ is $2700 \mathrm{~nm}^{2}$ (from such experiments we can estimate only the product $D t$ ). The diffusion constant $D$ of nitrogen in liquid iron at a mean temperature of the melt $(\sim 2500 \mathrm{~K})$ can be evaluated according to the relation $D=D_{0} \exp (-Q / R T)$, where $D_{0}=2.86 \times 10^{-3} \mathrm{~cm}^{2} / \mathrm{s}$ and $Q=61090 \mathrm{~J} / \mathrm{mol}$ [105], obtaining $D=15 \mathrm{~nm}^{2} / \mathrm{ns}$ and therefore $t_{e} \sim 180 \mathrm{~ns}$. Within the theory of Laser Supported Absorption (LSA) waves [31, 106], this time is consistent with the existence of a rarefaction fan and it represents the interaction time during which $\mathrm{N}$ is supplied to the target. The value of $D t_{g}$ is $4500 \mathrm{~nm}^{2}$, corresponding to a time $t_{g} \sim 300 \mathrm{~ns}$, that is in excellent agreement with the simulated duration of the melt (see Fig. 3.2). The fact that $t_{g} \neq t_{e}$ also reveals the different nature of the first pulse with respect to its evolution, meaning that the nitrogen take-up (erfc) and the further diffusion (gaussian) are distinct processes. A second series of isotopic experiments, 4/1*, 8/1*,32/1*,64/1* [29], was performed in order to check how the nitrogen take-up of the last pulse is affected by the nitrogen content. We observed that the erfc can nicely fit these profiles as well, but the surface concentration $C$ seems to increase with $n$, while the diffusion length $\sqrt{4 D t_{e}}$ decreases. The number of pulses $n$ is not the proper physical parameter, since the observed changes should be correlated to the prior modifications induced in the target. The total nitrogen concentration $S_{n-1}$ on the surface of the target, due to all previous $n-1$ pulses seems to be a suitable choice. We can express the evolution of $C$ as $C_{n}=C\left(S_{n-1}\right)$ and the evolution of $D t_{e}$ as $\left(D t_{e}\right)_{n}=D t_{e}\left(S_{n-1}\right)$ (notice that here $C_{n}$ represents the contribution of the $n^{\text {th }}$ erfc alone to the nitrogen surface concentration, while $S_{n-1}$ is the total nitrogen surface content after $n-1$ pulses); writing $C_{n}$ as a power series and considering only the linear term we obtain:

$$
C_{n}=a+b S_{n-1}=C\left(1+\sigma S_{n-1}\right)
$$


with $\sigma>0$ (since $C_{n}$ increases with $\left.S_{n-1}\right)$. In a similar way:

$$
\left(D t_{e}\right)_{n}=D t_{e}\left(1+\mu S_{n-1}\right)
$$

with $\mu<0$. $\sigma$ and $\mu$ are parameters of the model, while $S_{n-1}$ is determined by iteration. The dependencies of the diffusion length $2 \sqrt{\left(D t_{e}\right)_{n}}$ and the erfc surface concentration $C_{n}$ on the number of laser shots $n$ are reported in Fig. 5.3 .



Figure 5.3: Evolution of the erfc surface concentration $C_{n}$ and the erfc diffusion length $\left(D t_{e}\right)_{n}$ as a function of the number of laser pulses.

\subsubsection{The melting depth}

A factor that we have to include in the model is the finite melting depth. According to the thermodynamic simulations, the melting depth $d_{m}$ induced by the excimer laser in iron should be of the order of $1 \mu \mathrm{m}$ (see Fig. 3.2). We are assuming that the diffusion of nitrogen takes place only in the liquid iron (due to the much smaller nitrogen diffusion constant in the solid [105], the observed nitrogen profiles would not be justifiable). The amount of nitrogen is therefore confined inside the molten layer; mathematically this condition is satisfied with a proper choice of the gaussian normalization factor (the erfc is always within the melting depth), so Eq. (5.2) can be written as:

$$
g_{n}\left(z, t_{g}\right)=\frac{M}{\int_{0}^{d_{m}} \exp \left[-z^{\prime 2} / L_{n}^{2}\right] d z^{\prime}} \exp \left[-z^{2} / L_{n}^{2}\right] .
$$

The integral of $g_{n}\left(z, t_{g}\right)$ over the whole melting region $\left(0 \leq z \leq d_{m}\right)$ is $M$ for any values of $L_{n}$. We verified numerically that the condition reported in Eq. (5.7) is analogous to the introduction of a reflecting interface at the maximum melting depth: $\left.\frac{\partial C}{\partial z}\right|_{z=d_{m}}=0$. 


\subsubsection{The nitrogen losses}

The effect of each irradiation is not only to incorporate nitrogen in the sample as an erfc profile, but also to remove some of the nitrogen introduced by the previous pulses. This is attributed to the combined effects of ablation and nitrogen degassing of the target. The amount of the nitrogen incorporated in the sample with the $n^{\text {th }}$ erfc is:

$$
A_{n}=\int_{0}^{d_{m}} C_{n} \operatorname{erfc}\left[z / \sqrt{4\left(D t_{e}\right)_{n}}\right] d z \simeq 2 C_{n} \sqrt{\left(D t_{e}\right)_{n} / \pi},
$$

and what is remaining after the following irradiation can be written as:

$$
M_{n}^{n+1}=\int_{\Delta}^{d_{m}} C_{n} \operatorname{erfc}\left[z / \sqrt{4\left(D t_{e}\right)_{n}}\right] d z,
$$

where $\Delta$ is the nitrogen depletion depth of the erfc. Here, the parameter $\Delta$ includes both the ablation and the nitrogen degassing effects. $M_{n}^{n+1}$ represents the area of the gaussian evolution of the $n^{\text {th }}$ erfc after the $(n+1)^{\text {th }}$ pulse. Under the effect of further laser shots, the width of this gaussian profile will increase according to Eq. (5.3), but the area will decrease after each pulse due to ablation/degassing, and the nitrogen loss can be computed iteratively as follows:

$$
M_{n}^{q+1}=\int_{\delta}^{d_{m}}\left(\frac{M_{n}^{q}}{\int_{0}^{d_{m}} \exp \left[-z^{\prime 2} / L_{n}^{2}\right] d z^{\prime}} \exp \left[-z^{2} / L_{n}^{2}\right]\right) d z,
$$

where $\delta$ is the depletion depth of the gaussian and $q>n+1$. Both $\delta$ and $\Delta$ are parameters of the model determining the nitrogen loss per pulse. We observed that $\Delta>\delta$, i.e., the nitrogen loss is larger for the erfc than the gaussian. The value of $\delta$ is in good agreement with the ablation depth observed experimentally for the homogenized beam treatments [107], and the fact that $\Delta>\delta$ indicates a stronger degassing effect of the $\mathrm{N}$ in the erfc profile, probably correlated to the differences in the two types of nitrogen distributions (for example, the degassing rate has a quadratic dependence on the $\mathrm{N}$ concentration [105]).

\subsubsection{The results}

To summarize the results, a laser pulse "introduces" nitrogen in the sample, producing an erfc depth profile. Under the effect of the subsequent pulses the erfc evolves into gaussian profiles with increasing width and decreasing area vs. $n$ (using an erfc as initial condition in Eq. (5.4), the time evolution closely resembles a gaussian profile [108]), but a new erfc is created by each further laser shot. Therefore, the overall nitrogen profile after $n$ pulses is given by the sum of $n-1$ gaussians plus an erfc representing the effect of the $n^{\text {th }}$ pulse. Fig. 5.4 illustrates the experimental nitrogen profile after 8 pulses and its simulation. The contribution of each pulse is labeled in chronological order from 1 to 8 and added to all previous ones. The last pulse of a series of $n$ is hence described by the following erfc:

$$
e_{n}\left(z, t_{e}\right)=C_{n} \operatorname{erfc}\left[z / \sqrt{4\left(D t_{e}\right)_{n}}\right],
$$

where $C_{n}$ and $\left(D t_{e}\right)_{n}$ are calculated respectively from Eq. (5.5) and Eq. (5.6). The evolution of a previous erfc, for example the $j^{\text {th }}$ erfc (with $1 \leq j<n$ ), is given by the following 


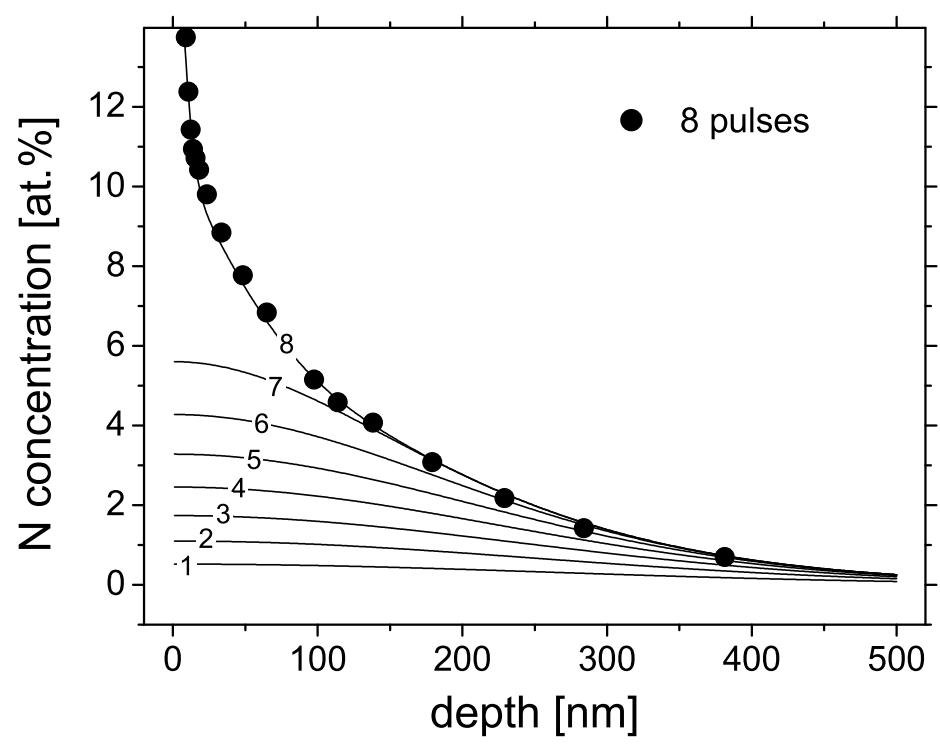

Figure 5.4: The nitrogen profile after 8 laser pulses (solid symbols): the evolution of each pulse is labeled in chronological order and added to all the previous ones.

gaussian:

$$
\begin{aligned}
g_{n}^{j}\left(z, t_{g}\right)= & \left(\frac{M_{j}^{n}}{\int_{0}^{d_{m}} \exp \left[-z^{\prime 2} /\left(4\left(D t_{e}\right)_{j}+4(n-j) D t_{g}\right)\right] d z^{\prime}}\right) \\
& \times \exp \left[-z^{2} /\left(4\left(D t_{e}\right)_{j}+4(n-j) D t_{g}\right)\right]
\end{aligned}
$$

where the value of $M_{j}^{n}$ is calculated according to Eq. (5.9) and Eq. (5.10), while the

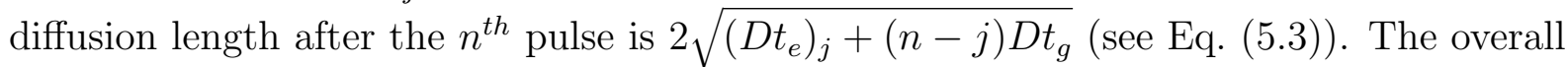
nitrogen depth profile $p_{n}$ after $n$ laser shots is described by the sum:

$$
p_{n}(z)=e_{n}\left(z, t_{e}\right)+\sum_{j=1}^{n-1} g_{n}^{j}\left(z, t_{g}\right) .
$$

Notice that the value of $S_{n-1}$ appearing in Eq. (5.5) and in Eq. (5.6) is simply:

$$
S_{n-1}=\left.p_{n-1}(z)\right|_{z=0}=C_{n-1}+\sum_{j=1}^{n-2} g_{n-1}^{j}\left(0, t_{g}\right) \text {. }
$$

Besides, the overall nitrogen loss $G_{n}$ after the $n^{\text {th }}$ pulse is:

$$
G_{n}=\int_{0}^{\Delta} e_{n}\left(z, t_{e}\right) d z+\int_{0}^{\delta} \sum_{j=1}^{n-1} g_{n}^{j}\left(z, t_{g}\right) d z \simeq \int_{0}^{\Delta} e_{n}\left(z, t_{e}\right) d z+\left(S_{n}-C_{n}\right) \delta .
$$

As shown in Fig. 5.5, the nitrogen take-up $A_{n}$ (Eq. (5.8) ) decreases with increasing number of laser pulses $n$, while the nitrogen loss $G_{n}$ increases with $n$. Both functions approach the same value in the limit $n \rightarrow \infty$, reflecting the fact that the nitrogen losses compensate the 


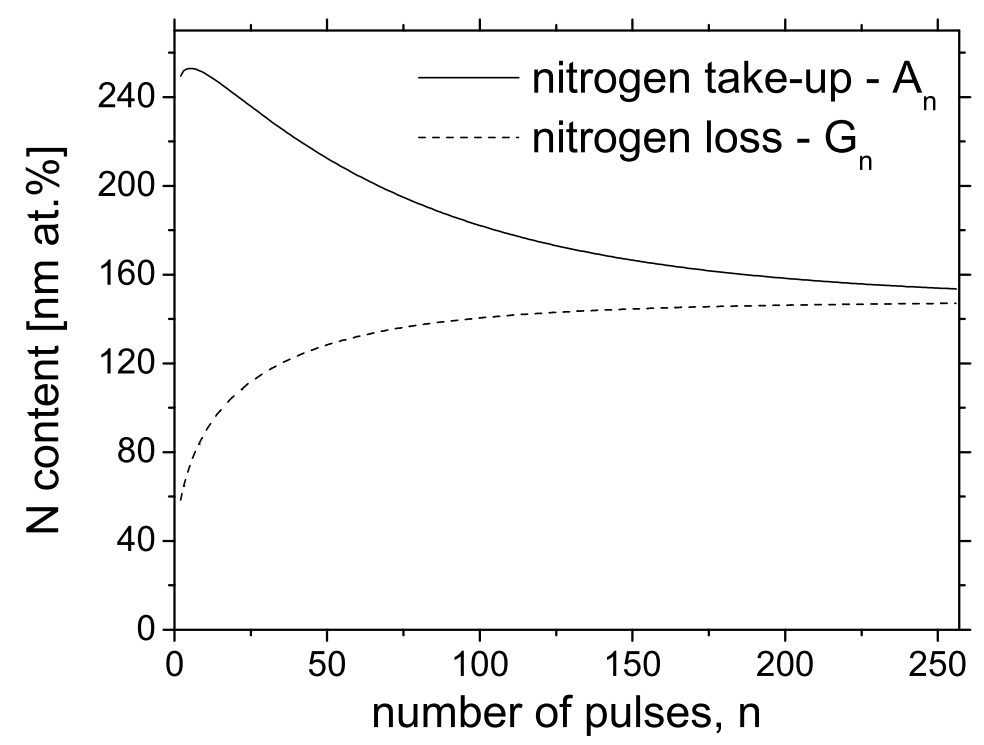

Figure 5.5: Nitrogen take-up (continuous line) and nitrogen losses (dashed line) per pulse as a function of the number of laser shots.

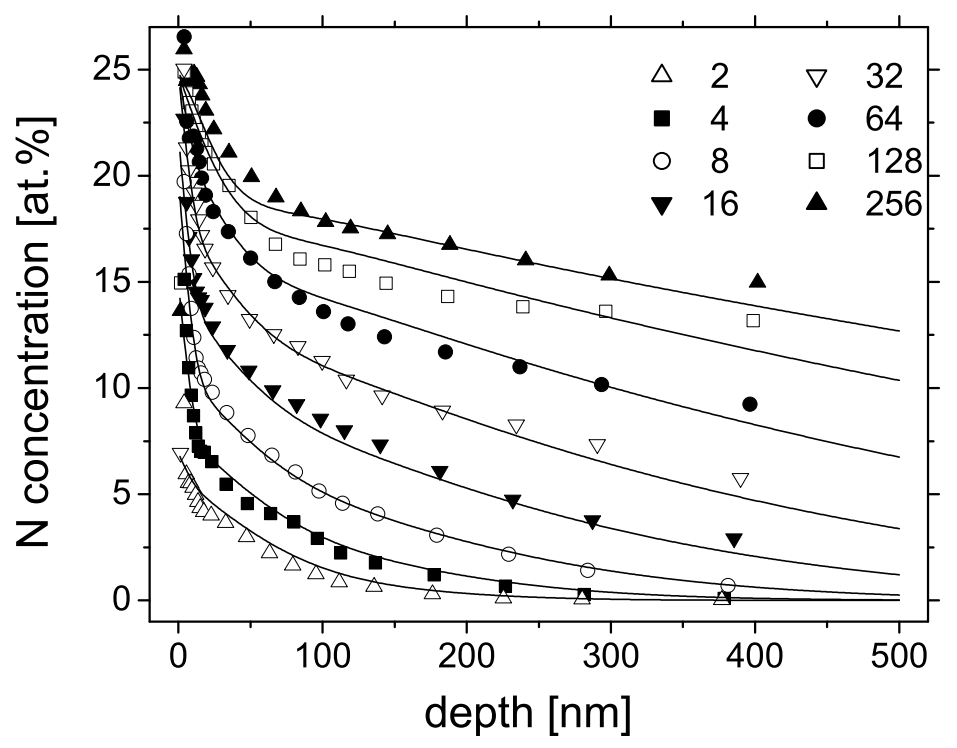

Figure 5.6: Experimental nitrogen depth profiles (symbols) and corresponding simulations according to Eq. 5.13 (solid lines) of the pulse series 2, 4, 8, 16, 32, 64, 128, 256.

up-take for high number of laser shots, and the overall nitrogen content remains constant. Eq. (5.13) is used to fit the experimental nitrogen depth profiles obtained with various number of pulses and the results are illustrated in Fig. 5.6. The simulations (solid lines) of the nitrogen profiles obtained with 2, 4, 8, 16, 32, 64, 128 and 256 laser pulses are 
calculated using the parameters reported in Table 5.1 .

Table 5.1: List of parameters used in the model. Where possible, a comparison with the experimental data has been given.

\begin{tabular}{lrr}
\hline \hline Parameter & Value & From [31, [107] \\
\hline$C[$ at.\%] & 4.1 & - \\
$D t_{e}\left[\mathrm{~nm}^{2}\right]$ & 2700 & 1700 \\
$\sigma\left[\right.$ at. $\left.\%^{-1}\right]$ & $1 / 35$ & - \\
$\mu\left[\right.$ at. $\left.\%^{-1}\right]$ & $-1 / 30$ & - \\
$D t_{g}\left[\mathrm{~nm}^{2}\right]$ & 4500 & 4500 \\
$\Delta[\mathrm{nm}]$ & 13 & - \\
$\delta[\mathrm{nm}]$ & 4 & 2 \\
$d_{m}[\mathrm{~nm}]$ & 900 & 800 \\
\hline \hline
\end{tabular}

\subsection{Laser irradiation in methane atmosphere}

After the successful and promising results of iron laser nitriding, the irradiation has been extended to other gaseous environments. Due to the technological importance of carbon in iron, a carbon-containing atmosphere was chosen. The following sections will focus on the results of the meandering irradiation in methane $\left(\mathrm{CH}_{4}\right)$ gas and the influence of experimental parameters, such as the spot overlap and the gas pressure on the mechanical and crystallographic properties will be illustrated.

\subsubsection{Influence of the meandering spot overlap}

The preliminary investigation on the efficiency of the carbon incorporation has been performed with fixed laser fluence $\left(4 \mathrm{~J} / \mathrm{cm}^{2}\right)$ and fixed methane gas pressure $(1.5 \mathrm{bar})$, varying the spot overlaps of the meandering treatment. The phase analysis has been carried out by means of Mössbauer spectroscopy (CEMS and CXMS) and XRD, the carbon depth profiles were investigated with RBS and the mechanical properties by nanoindentation. The CEM spectra of the samples treated with $8 \times 8,11 \times 12$ and $16 \times 16$ meander scans are shown in Fig. 5.7. The $8 \times 8$ scan produces a mixture of $\gamma$-Fe(C) (austenite), $\epsilon-\mathrm{Fe}_{x} \mathrm{C}$ and non-reacted $\alpha$-Fe. The $\gamma$ phase is fitted using a singlet (IS $\simeq 0.06(1) \mathrm{mm} / \mathrm{s}) \mathrm{rep}-$ resenting an iron site without carbon neighbors, and a doublet $(\mathrm{IS} \simeq 0.02(1) \mathrm{mm} / \mathrm{s}$, $\mathrm{QS} \simeq 0.65(2) \mathrm{mm} / \mathrm{s})$ due to an iron site with one carbon nearest neighbor. Using the binomial distribution, the relative area of the singlet, $A_{s}$, and the doublet, $A_{d}$, can be related to the occupancy of the interstitial octahedral sites, and the average carbon content $y_{c}$ can be estimated according to the relations [31]:

$$
y_{c}=1-\sqrt[6]{\frac{A_{s}}{A_{s}+A_{d}}},
$$




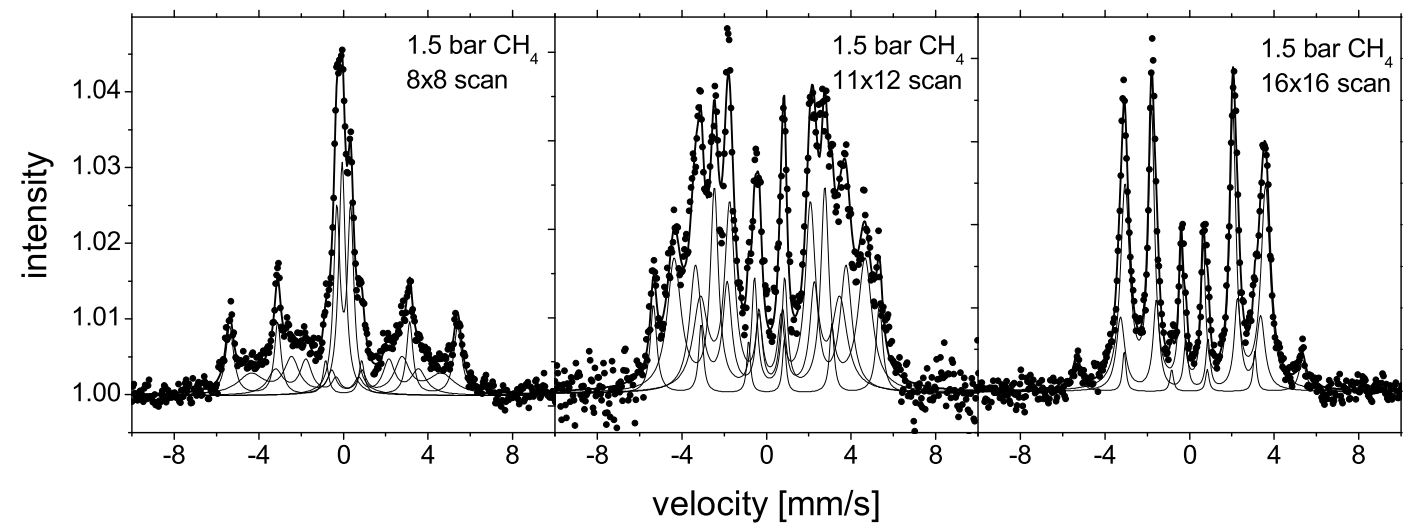

Figure 5.7: CEM spectra and corresponding fits of the iron samples irradiated in 1.5 bar $\mathrm{CH}_{4}$ with $8 \times 8,11 \times 12$ and $16 \times 16$ spot overlaps (from left to right).

obtaining $y_{c} \simeq 13$ at.\%. This value is higher than the maximum solubility of carbon in $\gamma$-Fe under equilibrium conditions, but as we explained in chapter 3 , the laser irradiation produces extreme pressures and temperature gradients that can lead to far-fromequilibrium solid solutions. The $\epsilon-\mathrm{Fe}_{x} \mathrm{C}$ is represented by two sextets with hyperfine fields of $\sim 28 \mathrm{~T}$ and $\sim 21 \mathrm{~T}$. They are attributed to the hexagonal arrangement of iron atoms with one $\left(\epsilon-\mathrm{Fe}_{6} \mathrm{C}\right)$ and two $\left(\epsilon-\mathrm{Fe}_{3} \mathrm{C}\right)$ interstitial carbon atoms, respectively. The relative fraction of the two components allows to determine the average stoichiometry of the phase, obtaining $x=4.33$. The atomic arrangement of the $\epsilon-\mathrm{Fe}_{3} \mathrm{C}$ carbide is shown in Fig. 5.8 the $\epsilon$ phase with the $\mathrm{Fe}_{6} \mathrm{C}$ stoichiometry is obtained removing one of the two carbon atoms. With the $11 \times 12$ meander scan, no austenite is observable with CEMS,

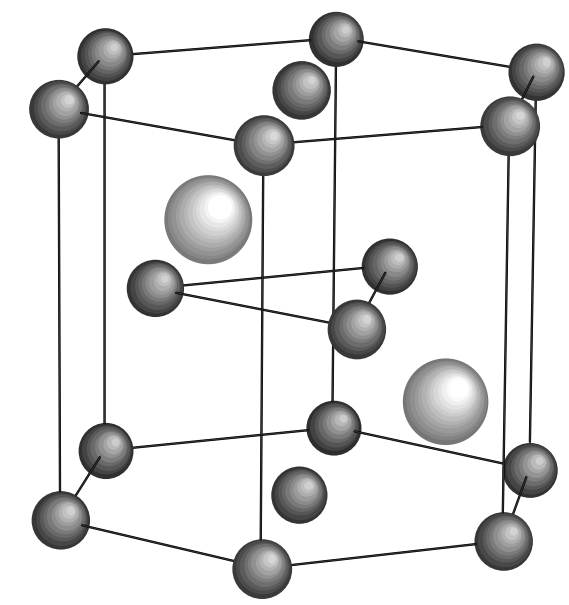

Figure 5.8: Atomic arrangements of the $\epsilon-\mathrm{Fe}_{3} \mathrm{C}$ carbide: the darker spots represent the positions of the iron atoms (space group $\mathrm{P} 6322$ ).

although a mixture of $\gamma$ and $\epsilon$ phases is visible with GIXRD (grazing incidence of $5^{\circ}$ ), 
as shown in Fig. 5.9. Since the information depth of GIXRD with the incidence angle of
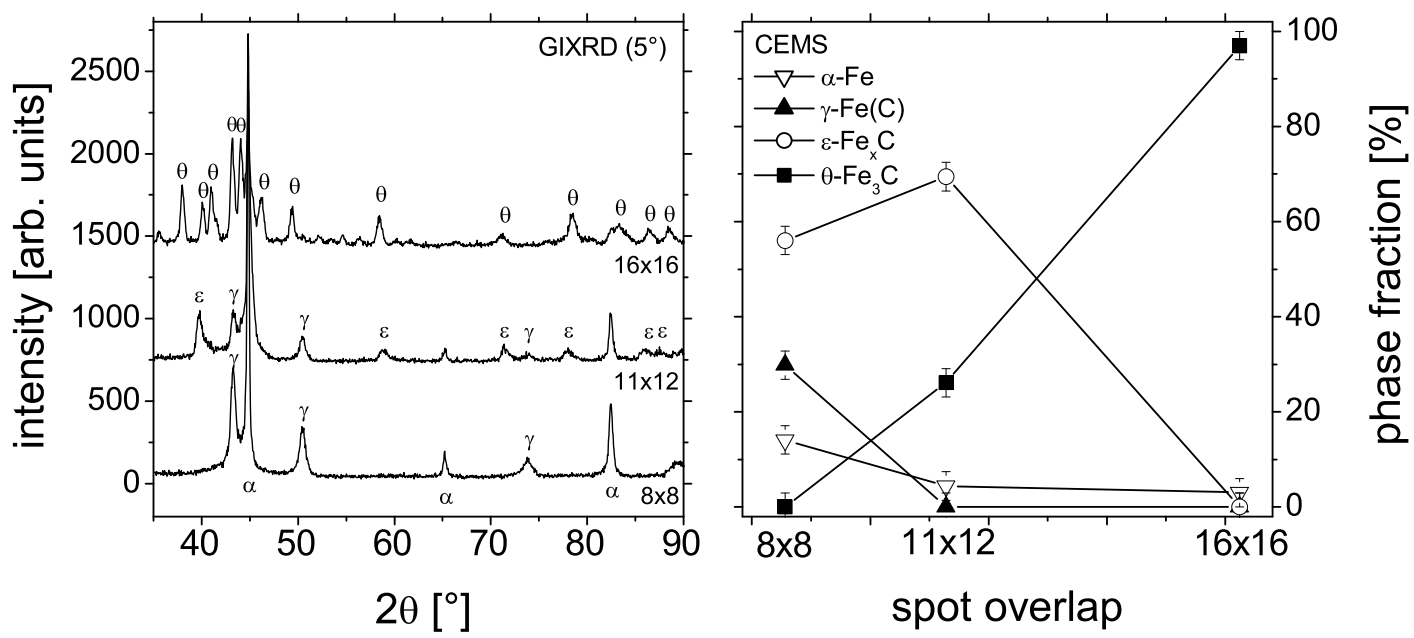

Figure 5.9: Phase fraction vs. the spot overlap as obtained from the CEMS analysis (right) and the corresponding GIXRD diffractograms measured at grazing angle of $5^{\circ}$ (left). All main XRD peaks are labeled.

$5^{\circ}$ is about $0.8 \mu \mathrm{m}$ (see Table 4.6), while CEMS is sensitive to the first $150 \mathrm{~nm}$ from the surface, we can conclude that the austenite is not formed at the surface of the sample, but deeper inside, most probably close to the maximum melting depth, where the carbon content is lower. Opposite to the austenite, some $\theta-\mathrm{Fe}_{3} \mathrm{C}$ appears in the CEM spectrum, but not in the X-ray diffractogram, indicating that its formation is limited to the surface (first $150 \mathrm{~nm}$ ) of the specimen. Notice that in the sample meandered with the $8 \times 8$ scan, only the austenite is visible with XRD, while both the the $\gamma$ and the $\epsilon$ phases are observed in the CEM spectra, suggesting that the latter is mainly confined on the sample surface. The treatment performed with the $16 \times 16$ spot overlap reveals the formation of a cementite $\left(\theta-\mathrm{Fe}_{3} \mathrm{C}\right)$ layer and no other carbide is observed by means of CEMS and GIXRD. The phase evolution as a function of the spot overlap is reported in Fig. 5.9. The Mössbauer spectral area has been converted to phase fraction taking into account the recoilless factor $f_{D W}$ of each phase, as given in Eq. 4.30). The Debye temperature of the carbon-rich carbides $(\epsilon$ and $\theta$ ) was measured by Mathalone et al. [109], who obtained the value of about $200 \mathrm{~K}$, corresponding to $f_{D W}=0.36$ at room temperature. This is quite small compared to the recoilless factors of $\alpha$-Fe (0.8) and $\gamma$-Fe (0.6). The mechanical properties of the irradiated samples have been investigated by nanoindentation, and the hardness curves are reported in Fig. 5.10. Jönsson and Hogmark [110] developed a model to estimate the composite hardness $H(d)$ of a coating on a softer substrate as a function of the indentation depth. According to their analysis:

$$
H(d)=H_{s}+\left(H_{c}-H_{s}\right)\left[2 k \frac{t}{d}-k^{2}\left(\frac{t}{d}\right)^{2}\right] .
$$

$H_{c}$ and $H_{s}$ are respectively the hardness of the coating and the substrate, $d$ is the indentation depth, $t$ is the coating thickness and $k$ (with $0.073<k<0.14$ ) is a parameter 


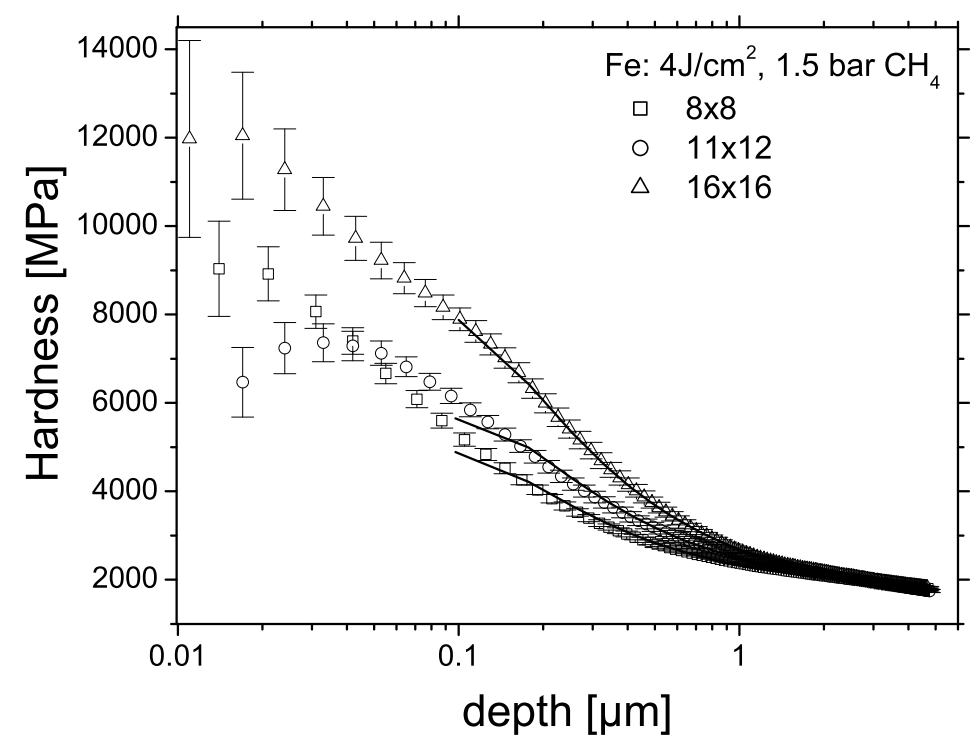

Figure 5.10: Microhardness curves of the samples meandered in $\mathrm{CH}_{4}$ (symbols). The continuous lines are the fitting according to Eq. 5.17.

describing the plastic deformation of the film as the diamond tip indents the sample. For simplicity we assumed $k=0.1$. The model works properly if the ratio $d / t>0.1$; since the thickness $t$ of the carburized layer should not exceed the melting depth $(\simeq 1 \mu \mathrm{m})$, the model can be applied to indentation depths $d>100 \mathrm{~nm}$. The continuous lines in Fig. 5.10 are the fits of the hardness profiles according to Eq. (5.17), using $H_{s}, H_{c}$ and $t$ as fitting parameters. The results are reported in Table 5.2. The substrate hardness

Table 5.2: Results the hardness profiles fits vs. the spot overlap.

\begin{tabular}{lrrr}
\hline \hline Meandering & $H_{s}[\mathrm{MPa}]$ & $H_{c}[\mathrm{MPa}]$ & $t[\mu \mathrm{m}]$ \\
\hline $8 \times 8$ & $1761(7)$ & $4888(85)$ & $0.95(3)$ \\
$11 \times 12$ & $1701(10)$ & $5668(70)$ & $1.04(3)$ \\
$16 \times 16$ & $1588(3)$ & $7927(20)$ & $0.91(1)$ \\
\hline \hline
\end{tabular}

$H_{s}$ is $\sim 1700 \mathrm{MPa}$ for all samples and the thickness $t$ of the layer is $\sim 0.95 \mu \mathrm{m}$, in good agreement with the calculated melting depth. The hardness $H_{c}$ of the carburized film increases with the spot overlap and therefore with the amount of cementite (the hardest carbide [39]). Despite the fact that the model was developed for homogeneous coatings on softer substrates, the fits are quite satisfactory and the average hardness of the carburized layers is consistent with the phase analysis. Since the mechanical properties and the crystallographic phases obtained with the $8 \times 8$ meander scan in $\mathrm{CH}_{4}$ closely resemble what has been observed with nitrogen gas under identical experimental conditions (same spot overlap, same pressure and laser fluence) [30, 111], the further investigation has been focused on the higher spot overlap. 


\subsubsection{Influence of the methane gas pressure}

As already pointed out in the previous section, with the $11 \times 12$ scan a complex overlap of magnetic phases has been observed with Mössbauer spectroscopy. In order to achieve a better understanding of the carburization process we performed the same meandering treatment at various methane pressures ranging from 0.1 bar to 10 bar. The Mössbauer spectra with the corresponding fits are shown in Fig. 5.11. The central component, at-

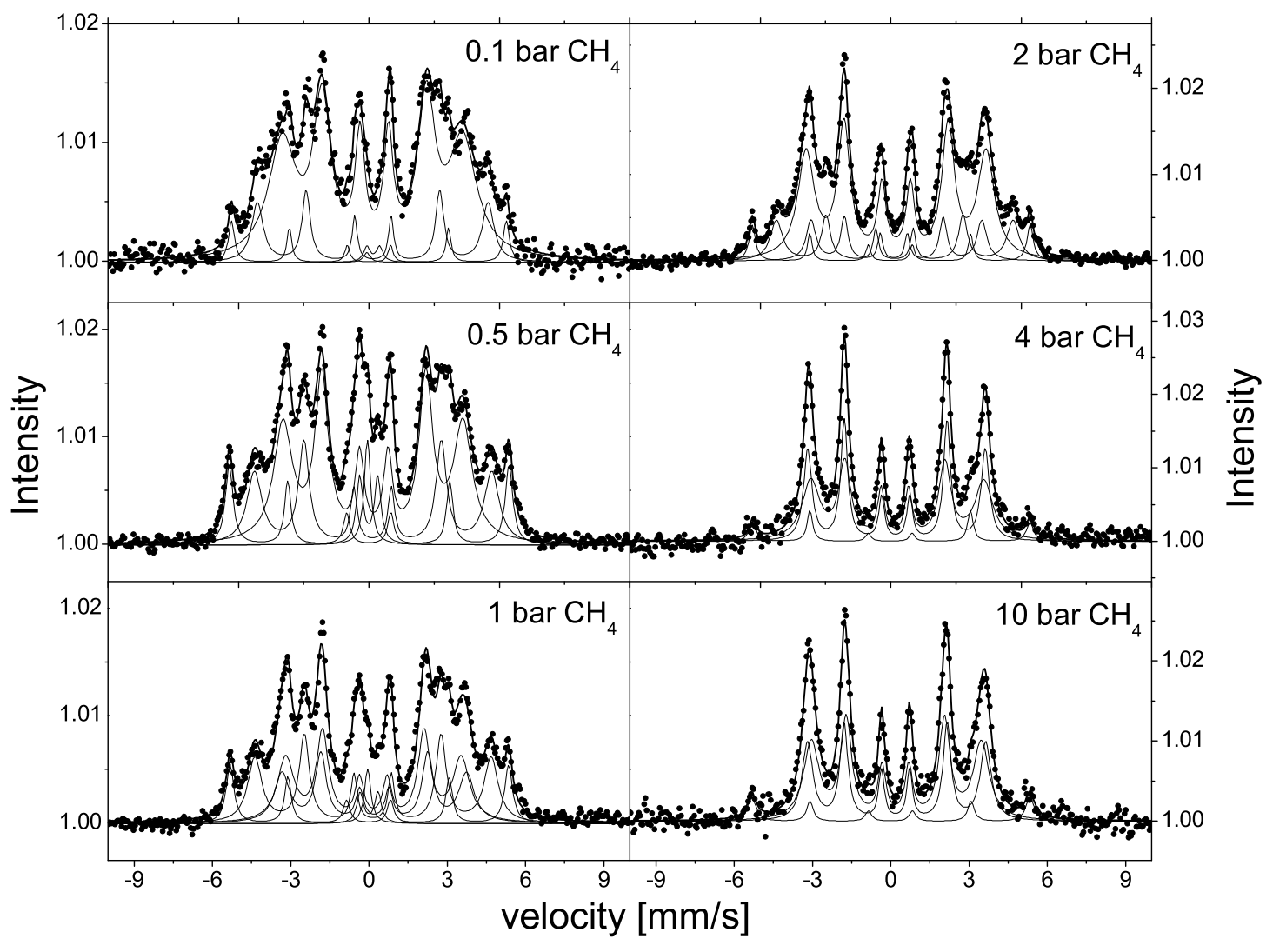

Figure 5.11: CEM spectra and corresponding fits of the iron samples irradiated at different methane pressures with the $11 \times 12$ meandered scan.

tributed to $\gamma-\mathrm{Fe}(\mathrm{C})$ austenite, is detectable only for pressures lower than 2 bar, while the sextet of non-reacted iron and additional broad magnetic components are observed over the entire pressure range. The two characteristics sextets of $\epsilon-\mathrm{Fe}_{x} \mathrm{C}$ carbide can be distinguished, but a gradual increase in the isomer shift of the lower hyperfine field component (corresponding to $\epsilon-\mathrm{Fe}_{3} \mathrm{C}$ ) is observed with increasing pressures. Simultaneously, the relative area of the higher hyperfine field component (corresponding to $\epsilon-\mathrm{Fe}_{6} \mathrm{C}$ ) gradually drops, disappearing if the $\mathrm{CH}_{4}$ pressure is higher than 4 bar. As confirmed by GIXRD measurements carried out at grazing incidence of $1^{\circ}$ (corresponding to an information depth of about $200 \mathrm{~nm}$ ), this behaviour is attributed to the gradual increase of the $\theta-\mathrm{Fe}_{3} \mathrm{C}$ relative fraction at expenses of the $\epsilon-\mathrm{Fe}_{x} \mathrm{C}$ phase with increasing methane 
pressure. The Mössbauer analysis has been performed employing two distinct subspectra for the lower hyperfine field component, constraining their isomer shift to $0.17 \mathrm{~mm} / \mathrm{s}$ for the $\epsilon-\mathrm{Fe}_{3} \mathrm{C}$ and to $0.205 \mathrm{~mm} / \mathrm{s}$ for the $\theta-\mathrm{Fe}_{3} \mathrm{C}$ phase. These are their values at the edges of the pressure range, i.e. the isomer shift of the $\epsilon-\mathrm{Fe}_{3} \mathrm{C}$ formed at $0.1 \mathrm{bar}^{\mathrm{CH}_{4}}$ (where no cementite is present) and the isomer shift of the $\theta-\mathrm{Fe}_{3} \mathrm{C}$ formed at 10 bar $\mathrm{CH}_{4}$ (where no $\epsilon$ carbide is present). This procedure allows to separate the $\epsilon$ and the $\theta$ phases in the Mössbauer spectra. At high methane pressure only the cementite is formed and the result is analogous to the irradiation in 1.5 bar $\mathrm{CH}_{4}$ with the $16 \times 16$ scan. The relative phase fractions extrapolated from the Mössbauer analysis are reported in Fig. 5.12 as a function of the $\mathrm{CH}_{4}$ pressure. The amount of non-reacted iron is about $5 \%$ regardless of

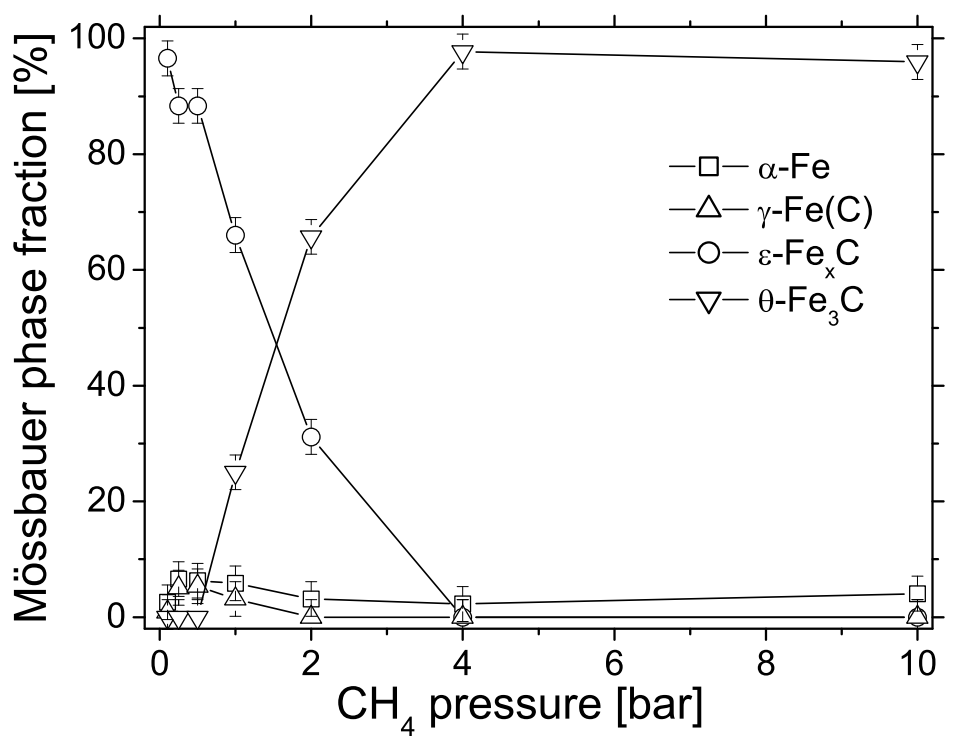

Figure 5.12: Phase fractions obtained from the Mössbauer analysis vs. the methane gas pressure.

the ambient pressure, while the amount of cementite sharply increases at expenses of the $\epsilon-\mathrm{Fe}_{x} \mathrm{C}$ carbide in the region 1-4 bar. The austenitic phase drops simultaneously to the $\epsilon$ phase and both are not observed for irradiations performed at methane pressures higher than 2 bar. Thanks to the capability of the Mössbauer spectroscopy not only the relative amount of all phases can be obtained, but also the average carbon content in each of them. The overall concentration of carbon as a function of the methane pressure is reported in Fig. 5.13. The data have been compared with the carbon contents extrapolated from the RBS depth profiles (averaged over the first $150 \mathrm{~nm}$ from the surface in order to compare the results with the CEMS analysis). The agreement is quite good for pressures higher than 2 bar. The large discrepancy in the region $0.1-2$ bar is due to the considerable amount of carbon deposited on the samples treated at low pressure (see the RBS depth profiles in the inset of Fig. 5.13). Since the Mössbauer technique is not sensitive to carbon and the GIXRD measurements did not show any crystalline phase other than iron or iron carbides, we can conclude that the carbon forms amorphous layers. At the moment, a valid explanation of this phenomenon is missing. Intuitively, one would expect thicker 
carbon layers for increasing methane pressure, but the opposite trend is observed instead. This might be correlated to the shielding effect of the ambient gas on the laser energy (see section 3.3.3). The higher is the methane pressure, the stronger is the shielding of the laser beam and less laser energy reaches the substrate. This reduces the surface temperature and thus the vapor pressure. As a consequence, the effect of the substrate on the plasma formation is hindered: fewer $\mathrm{CH}_{4}$ molecules are ionized/decomposed, and less carbon is deposited on the target surface.

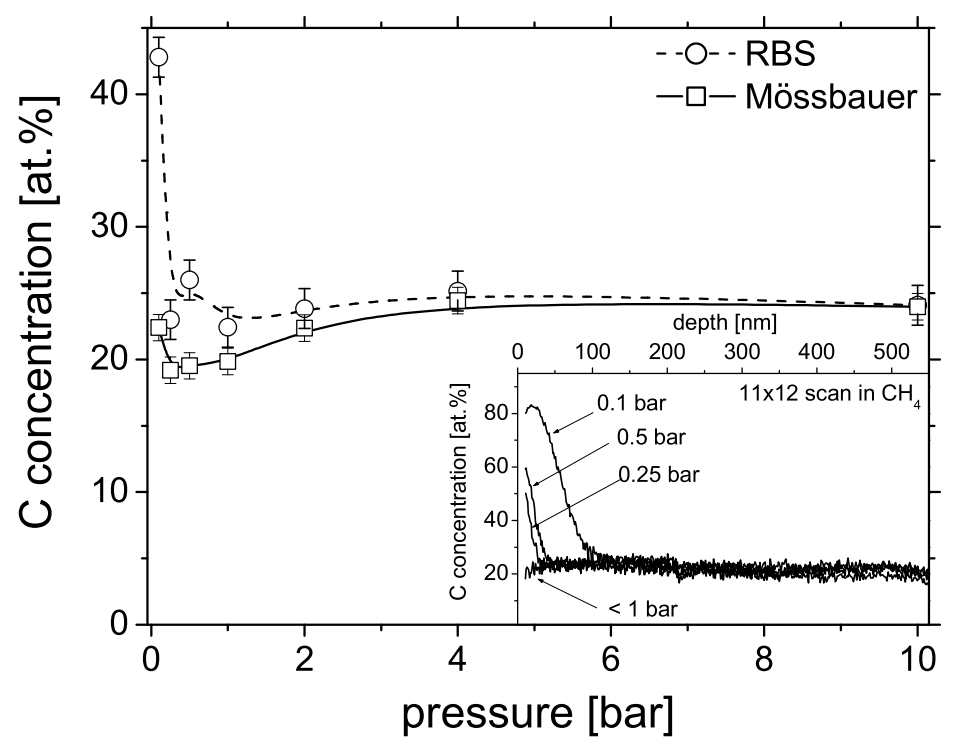

Figure 5.13: Average carbon content vs. the methane gas pressure obtained from the RBS depth profiles (dash line) and from the Mössbauer analysis (continuous line). The inset show the most significant RBS carbon depth profiles.

\subsubsection{Formation of a homogeneous cementite layer}

As we already mentioned in the preliminary investigation, with the $16 \times 16$ meander scan at an intermediate methane pressure $(1.5$ bar $)$, the only iron-carbide formed in the irradiated sample is $\theta-\mathrm{Fe}_{3} \mathrm{C}$. Due to its technological importance in iron metallurgy, a more detailed investigation has been carried out [102]. Fig. 5.14 shows the RBS spectra of the untreated substrate and the one irradiated at $4 \mathrm{~J} / \mathrm{cm}^{2}$ in 2 bar $\mathrm{CH}_{4}$ with the $16 \times 16$ scan. After the laser treatment, the Fe yield reduces due to the carbon incorporation into the specimen. The simulation performed with the RUMP code shows excellent agreement with the cementite stoichiometry $(25$ at.\% $\mathrm{C}$ and 75 at.\% Fe) to a depth of at least $650 \mathrm{~nm}$. The Mössbauer data and their analyses are shown in Fig. 5.15. The CEM spectrum, with an information depth of about $150 \mathrm{~nm}$, shows that almost $97 \%$ of the relative phase fraction is cementite, while the remaining part is non-reacted $\alpha$-Fe. The two characteristic sextets of $\theta-\mathrm{Fe}_{3} \mathrm{C}$ can be clearly distinguished in the spectrum. They originate from the two different crystallographic iron sites: $\mathrm{Fe}(\mathrm{I})$ with $3 \mathrm{C}$ and $11 \mathrm{Fe}$ 


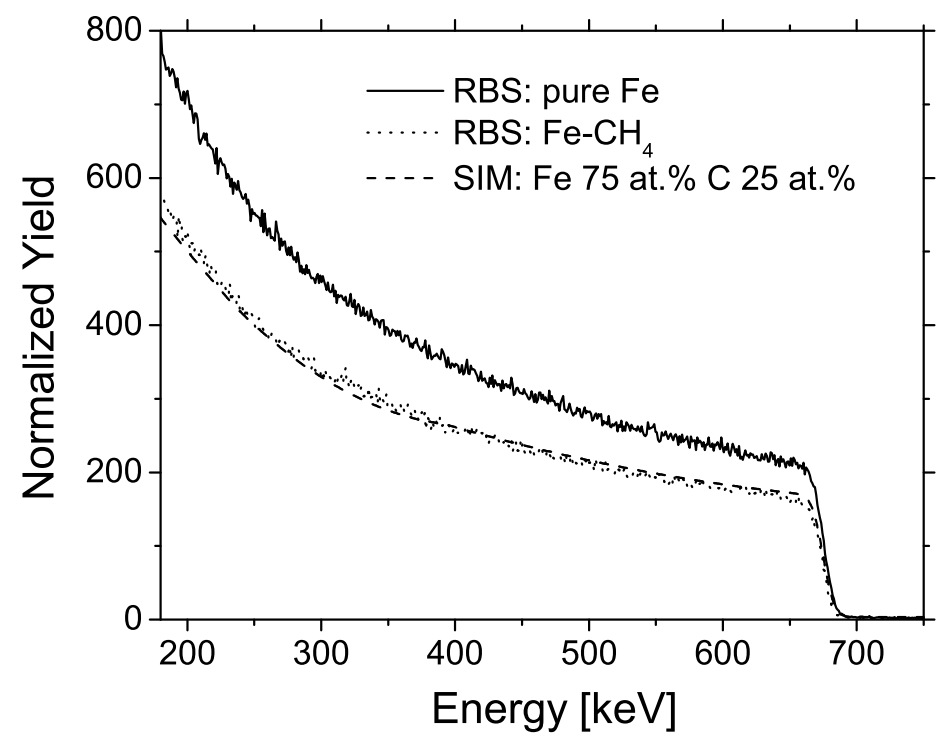

Figure 5.14: RBS spectra of the non-treated sample (higher yield) and the one irradiated with the $16 \times 16$ scan at fluence of $4 \mathrm{~J} / \mathrm{cm}^{2}$ in 2 bar $\mathrm{CH}_{4}$ (lower yield). The dashed line is the RUMP simulation of the cementite phase.

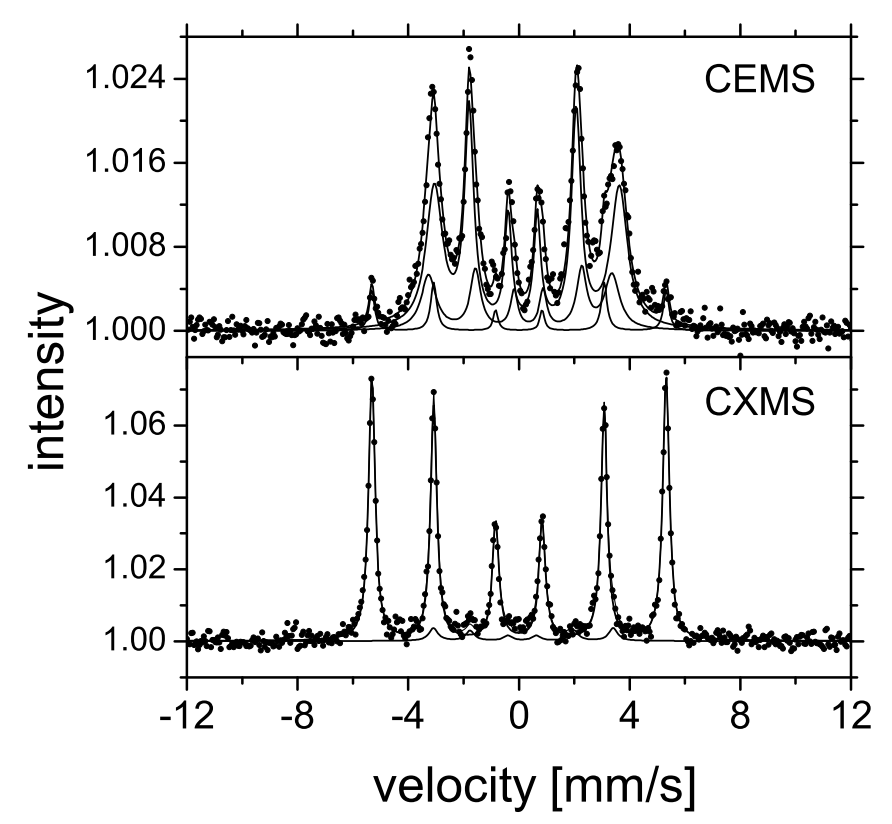

Figure 5.15: CEM (top) and CXM (bottom) spectra of the $16 \times 16$ meandered sample in 2 bar $\mathrm{CH}_{4}$.

nearest neighbors, and Fe(II) with $2 \mathrm{C}$ and 12 Fe nearest neighbors [39]. The CXM spectrum, with an information depth of the order of $10 \mu \mathrm{m}$, reveals that only about $10 \%$ 
of the relative phase fraction can be attributed to the carbide phase, indicating that the $\theta-\mathrm{Fe}_{3} \mathrm{C}$ layer is about $1 \mu \mathrm{m}$ thick, in agreement with the calculated melting depth. All hyperfine parameters are shown in Table 5.3 and agree well with those reported in the literature [112]. X-Ray diffraction was carried out at three different grazing incidence

Table 5.3: Mössbauer parameters of the spectra shown in Fig. 5.15.

\begin{tabular}{llrrrr}
\hline \hline & Phase & IS $[\mathrm{mm} / \mathrm{s}]$ & QS $[\mathrm{mm} / \mathrm{s}]$ & $\mathrm{HF}[\mathrm{T}]$ & fraction [\%] \\
\hline \multirow{3}{*}{ CEMS } & $\mathrm{Fe}_{3} \mathrm{C}(\mathrm{I})$ & 0.21 & 0.075 & 20.72 & $66(3)$ \\
& $\mathrm{Fe}{ }_{3} \mathrm{C}(\mathrm{II})$ & 0.20 & -0.15 & 20.56 & $31(2)$ \\
& $\alpha$-Fe & 0.0 & 0.0 & 33 & $3(1)$ \\
\hline \multirow{2}{*}{ CXMS } & $\mathrm{Fe}_{3} \mathrm{C}$ & 0.20 & 0.003 & 20.4 & $9.7(2)$ \\
& $\alpha$-Fe & 0.0 & 0.0 & 33 & $90.3(2)$ \\
\hline \hline
\end{tabular}

angles $\left(5^{\circ}, 10^{\circ}, 15^{\circ}\right)$ to obtain qualitative information on the crystallographic morphology of the carburized layer. The three indexed peaks in Fig. 5.16 correspond to the $\alpha$-Fe phase and originate from the non-reacted substrate, while all other peaks can be assigned to the $\theta-\mathrm{Fe}_{3} \mathrm{C}$ phase [44]. Since the cementite phase is at the surface of the sample, the relative

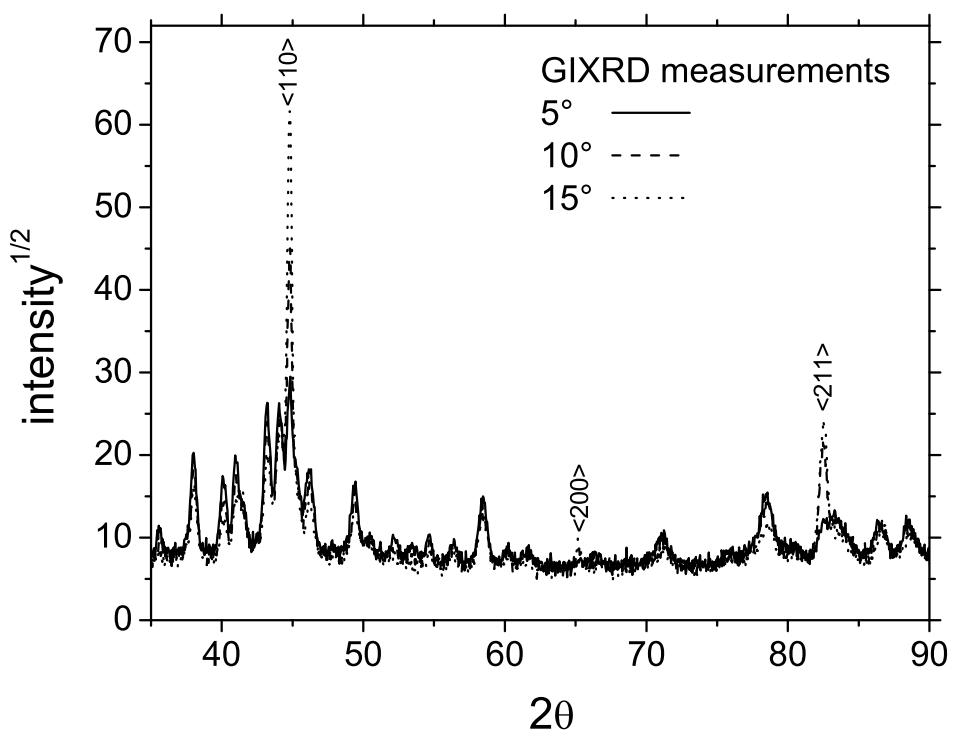

Figure 5.16: GIXRD diffractograms of the $16 \times 16$ scan in $\mathrm{CH}_{4}$ taken at three different grazing incidence angles $\left(5^{\circ}, 10^{\circ}, 15^{\circ}\right)$ : the indexed peaks correspond to the iron substrate, all other peaks belong to the cementite phase.

intensity of the $\mathrm{Fe}_{3} \mathrm{C}$ peaks decreases with increasing incidence angle, i.e. with increasing X-ray penetration depth. Besides, no other carbide can be detected at larger depth, in agreement with the CXMS measurement, indicating the good degree of homogeneity of the layer. The possible mechanism governing the formation of the cementite layer can be 
explained with the help of the $\mathrm{Fe}-\mathrm{C}$ temperature-carbon activity phase diagram reported in Fig. 5.17. Due to the high temperature reached during the laser irradiation (>4000 K



Figure 5.17: Temperature-carbon activity phase diagram of the Fe-C system (data from [42]).

at a laser fluence of $4 \mathrm{~J} / \mathrm{cm}^{2}$, see Fig. 3.2), the system, that initially is at the lower left-hand corner of the phase diagram, can rapidly move into the $\gamma$-Fe region. However, the high quenching rates $\left(\sim 10^{9} \mathrm{~K} / \mathrm{s}\right)$ obtained with excimer laser processes rapidly bring the system back to the $\alpha$-Fe region. As the number of pulses increases, more carbon is incorporated in the sample and the system gradually moves towards the higher carbon activity side. Eventually it may cross the steep $\gamma / \mathrm{Fe}_{3} \mathrm{C}$ phase boundary in the direction of the cementite, where no $\gamma$-Fe is present, cooling in the $\mathrm{Fe}_{3} \mathrm{C}$ region. When the carbon incorporated in the iron matrix has reached the value of 25 at.\%, the cementite phase will grow inside the iron substrate leading to the formation of a homogeneous $\mathrm{Fe}_{3} \mathrm{C}$ coating. The transition carbides, due to their unstable nature, are not included in the carbon activity phase diagram, but we observed in the previous section that the $\gamma-\mathrm{Fe}(\mathrm{C})$ phase is "coupled" to the $\epsilon$ phase (their existence is simultaneous) and the $\gamma$-Fe region in Fig. 5.17 might be correlated to the $\epsilon-\mathrm{Fe}_{x} \mathrm{C}$ phase (reported in square brackets). 


\section{Chapter 6}

\section{The aluminum substrate}

Laser nitriding of aluminum substrates has been studied in the last decades with particular emphasis on the morphological, structural and tribological properties [113, 114, 115], but so far no effort has been made to understand the mechanisms of nitrogen incorporation and mass transport. In order to investigate the influence of the experimental parameters on the nitriding efficiency and the mass transport, a systematic study on the effects of the laser irradiation of pure $\mathrm{Al}$ substrates in nitrogen atmosphere has been carried out, revealing the strong influence of the laser fluence on the mass transport properties [98, 116]. Even if aluminum carbide is technologically not as attractive as AlN, laser irradiation in methane atmosphere has been performed, demonstrating that the carbon incorporation is effective.

\subsection{Laser nitriding of aluminum}

The following sections will describe the results of the laser irradiation of aluminum substrates in nitrogen atmosphere as a function of the laser fluence, the number of pulses and the gas pressure. Special emphasis will be given to the mass transport mechanism, revealing how the migration of nitrogen is strongly correlated to the laser-induced temperature of the substrate.

\subsubsection{Preliminary investigations: the laser fluence dependence}

Fig. 6.1 depicts the nitrogen depth profiles of the Al samples irradiated as single spot with 64 laser pulses in 1 bar $\mathrm{N}_{2}$, at fluences $\phi$ ranging from 1 to $4 \mathrm{~J} / \mathrm{cm}^{2}$, in steps of $0.5 \mathrm{~J} / \mathrm{cm}^{2}$. Below $2 \mathrm{~J} / \mathrm{cm}^{2}$, the maximum nitrogen concentration is lower than 10 at.\% and the thickness of the nitrided layer is of the order of $100 \mathrm{~nm}$. Between 2 and $3 \mathrm{~J} / \mathrm{cm}^{2}$, diffusion-like profiles (i.e. exponential decays analogous to the nitrogen profiles in iron, see section 5.1) are observed: the nitrogen concentration at the surface is higher than 30 at.\% and the thickness of the nitrided layer exceeds $600 \mathrm{~nm}$. Since the nitriding efficiency can be measured by the mean nitrogen concentration and/or by the average thickness of the nitrided layer, the nitriding process in $\mathrm{Al}$ becomes effective at a laser 


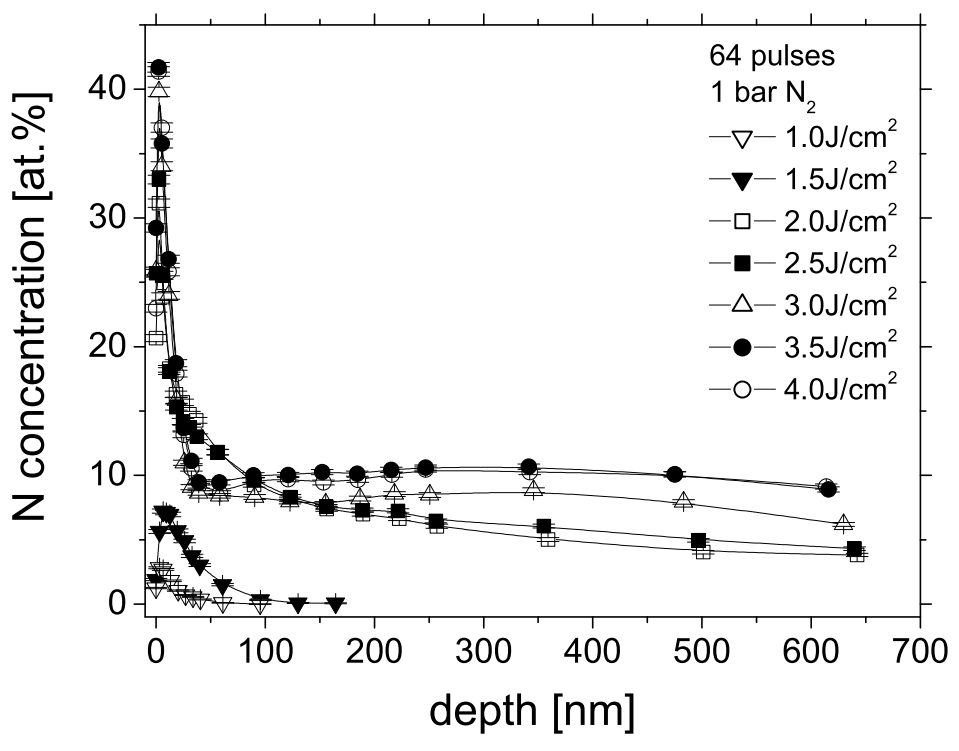

Figure 6.1: Nitrogen depth profiles obtained after irradiation with 64 pulses in 1 bar $\mathrm{N}_{2}$ at laser fluences ranging from 1 to $4 \mathrm{~J} / \mathrm{cm}^{2}$.

fluence of about $2 \mathrm{~J} / \mathrm{cm}^{2}$. Above $3 \mathrm{~J} / \mathrm{cm}^{2}$, roughly homogeneous nitrogen profiles are formed within the investigated depth. Sharp nitride peaks with an average thickness of a few tens of nanometers appear on the surface of these samples: they are attributed to the "fall-out" (plasma re-condensate) of AlN clusters, most probably formed in the laserinduced plasma. The supersaturated $\mathrm{Al}$ vapor on the sample surface might facilitate the chemical reaction between $\mathrm{Al}$ and $\mathrm{N}$ as already pointed out in section 3.3. In order to better understand the nature of the fluence dependence across $\phi=3 \mathrm{~J} / \mathrm{cm}^{2}$ we have studied in more detail the nitriding effect at $2 \mathrm{~J} / \mathrm{cm}^{2}$ and at $4 \mathrm{~J} / \mathrm{cm}^{2}$. The differences are strongly correlated with the maximum temperature induced in the irradiated sample. According to the thermal simulations, as reported in Fig. 6.2, at $4 \mathrm{~J} / \mathrm{cm}^{2}$ the $\mathrm{Al}$ surface reaches the temperature of $3800 \mathrm{~K}$ : this value is higher than the dissociation temperature of AlN (2400 K at 1 bar $\mathrm{N}_{2}$, but it increases with the ambient gas pressure [48]) and the melting point of AlN (3070 K [38]), while at $2 \mathrm{~J} / \mathrm{cm}^{2}$ the maximum surface temperature is about $2000 \mathrm{~K}$, i.e. higher than the melting point of pure $\mathrm{Al}$, but insufficient to dissolve the AlN. This aspect will be analyzed extensively in the following sections.

\subsubsection{Mass transport mechanism at $\phi=4 \mathbf{J} / \mathrm{cm}^{2}$}

The depth profiles of the samples irradiated at $4 \mathrm{~J} / \mathrm{cm}^{2}$ in 1 bar $\mathrm{N}_{2}$ are shown in Fig. 6.3. The nitrogen concentrations are rather homogeneous, increasing monotonically with the number of laser pulses up to the mean value of 14.7 at.\% after 256 shots. The average nitrogen content drastically increases if the irradiation at $4 \mathrm{~J} / \mathrm{cm}^{2}$ is performed in higher ambient gas pressure. Fig. 6.4 reports the nitrogen profiles obtained at $\phi=4 \mathrm{~J} / \mathrm{cm}^{2}$ in 6 bar $\mathrm{N}_{2}$. After 256 pulses, the homogeneous (within the experimental errors) $\mathrm{N}$ 


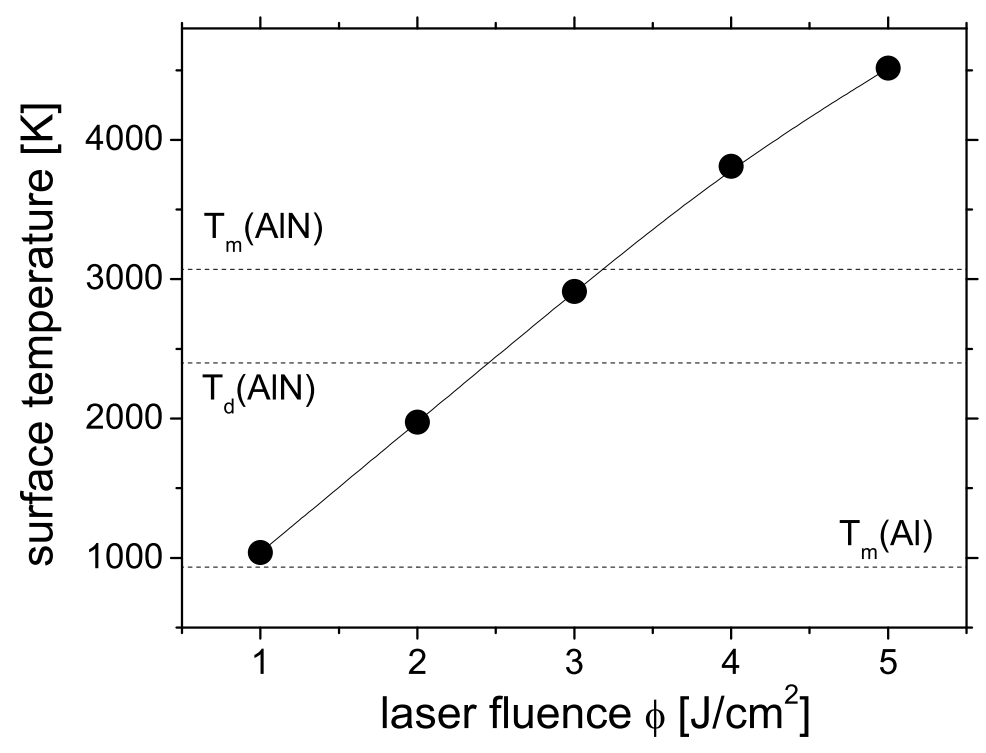

Figure 6.2: Simulation of the maximum Al surface temperature vs. the laser fluence. The dashed lines represent the melting point of $\mathrm{Al}$, the dissociation and the melting points of AlN, respectively (from bottom to top).

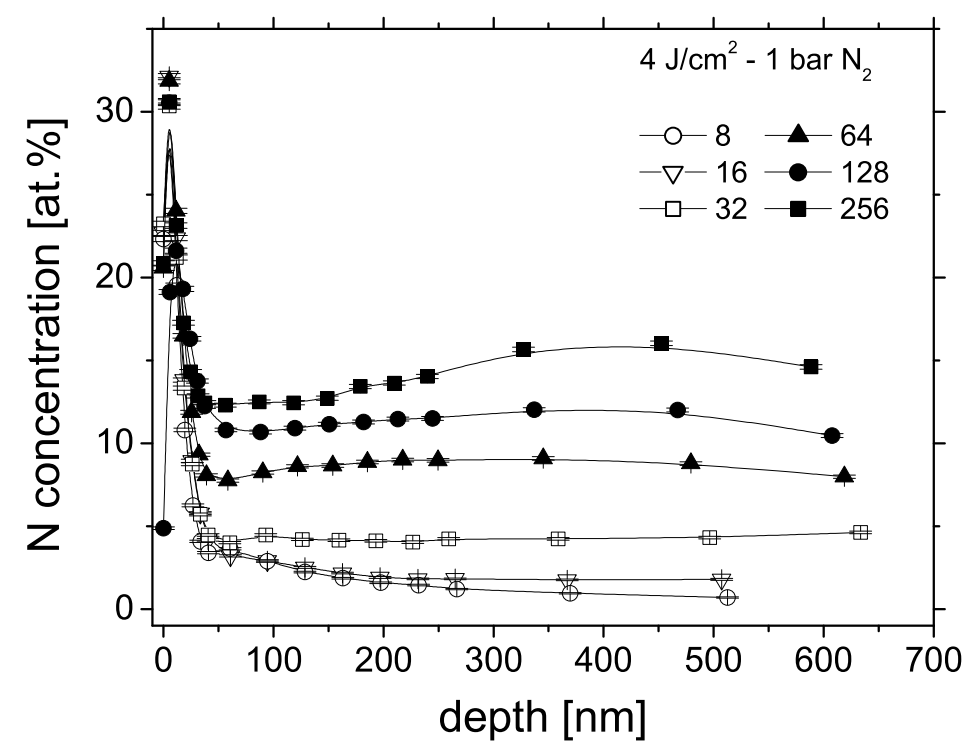

Figure 6.3: Nitrogen depth profiles after irradiation in 1 bar $\mathrm{N}_{2}$ at a fluence of $4 \mathrm{~J} / \mathrm{cm}^{2}$ and various numbers of laser pulses.

concentration reaches the value of $\sim 48$ at.\%, i.e. very close to the stoichiometry of AlN. According to the temperature simulations, at a laser fluence of $4 \mathrm{~J} / \mathrm{cm}^{2}$ the aluminum surface exceeds the melting point of AlN for about $80 \mathrm{~ns}$ and remains liquid for about 


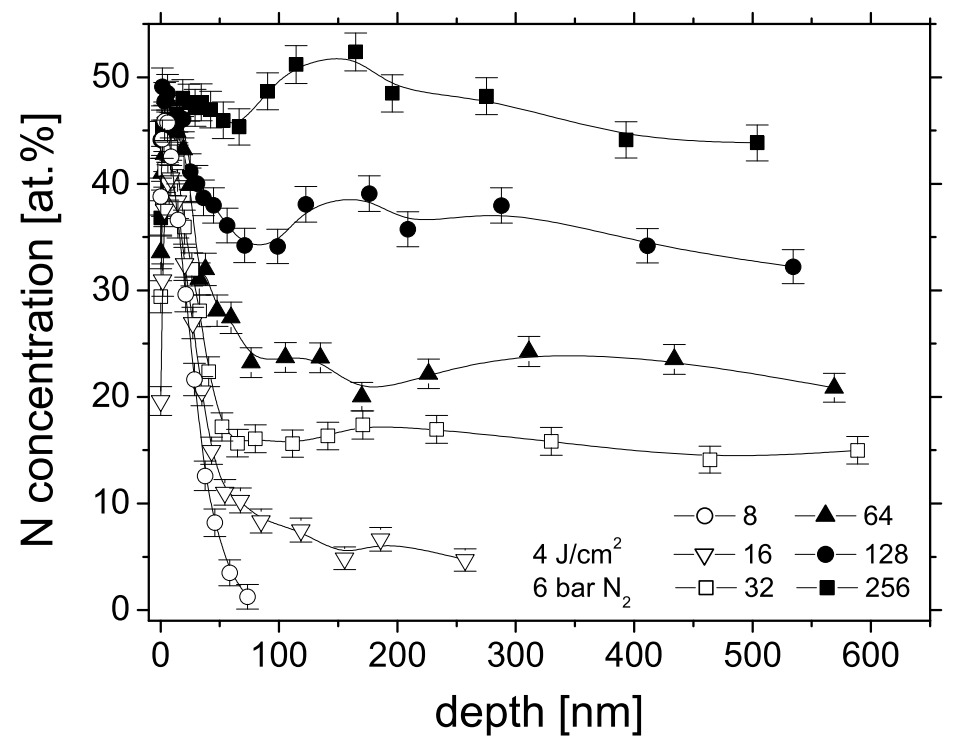

Figure 6.4: Nitrogen depth profiles after irradiation in 6 bar $\mathrm{N}_{2}$ at a fluence of $4 \mathrm{~J} / \mathrm{cm}^{2}$ and various numbers of laser pulses.

800 ns, as shown in Fig. 6.5. During the melting time, atomic nitrogen can be in solution

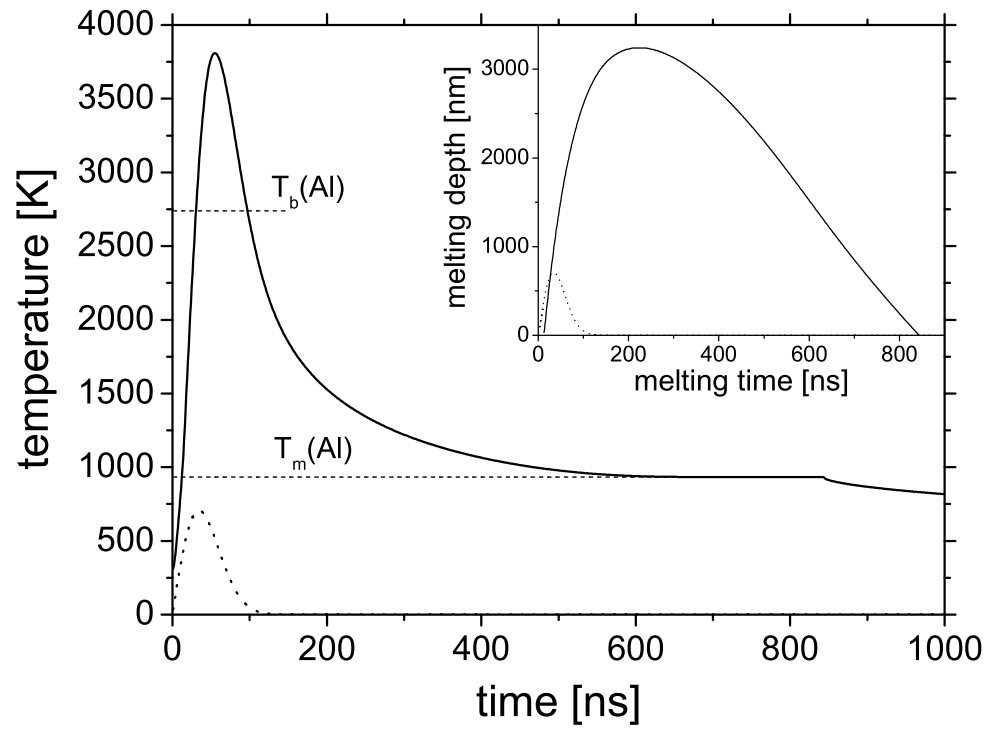

Figure 6.5: Time evolution of the surface temperature of pure $\mathrm{Al}$ irradiated at $4 \mathrm{~J} / \mathrm{cm}^{2}$. The inset shows the melting depth vs. the melting time (the dashed curves are the duration of the laser pulse).

in the liquid $\mathrm{Al}$ up to the solubility limit of few atomic percent (see Fig. 2.4), and can form 
AlN as the temperature of the liquid drops to the melting point of pure Al. When the solidification temperature is reached, all the nitrogen in the sample will be in the nitride form, since no N solubility is possible in solid Al. However, during the cooling of the liquid some atomic nitrogen may segregate [117] at the moving solid-liquid interface or it may form $\mathrm{N}_{2}$ molecules that will eventually outgas, being chemically inert and very stable [43]. The segregation process is strongly affected by the velocity $v_{s l}$ of the solid-liquid interface during the cooling (the slower is the solid/liquid interface, the stronger is the segregation, see section 7.2.2): according to the simulations, at $4 \mathrm{~J} / \mathrm{cm}^{2}$ the value of $v_{s l}$ is about $7 \mathrm{~m} / \mathrm{s}$, while at $2 \mathrm{~J} / \mathrm{cm}^{2}$ the calculation leads to $v_{s l} \sim 12 \mathrm{~m} / \mathrm{s}$ and the segregation should be less effective. The measured nitrogen depth profiles can be attributed to two distinct and competing effects: (i) an inward nitrogen flux due to the plasma-substrate coupling, leading to the nitrogen incorporation, and (ii) an outgassing flux due to the nitrogen segregation/recombination into $\mathrm{N}_{2}$ molecules. It is reasonable to assume that the average nitrogen take-up per pulse $S_{i n}$ [at.\% pulse ${ }^{-1}$ ] caused by the inward flux increases with the abundance of atomic nitrogen in the laser-induced plasma and therefore with the ambient gas pressure. On the other hand, the outgassing rate should scale with the nitrogen concentration $c_{N}$ [at.\%] inside the sample (the more nitrogen exceeds the solubility limit, the larger is the outgassing). Therefore the rate of nitrogen loss per pulse due to the outgassing flux can be written as $S_{\text {out }}=c_{N} / l$, where the proportionality constant $1 / l$ $\left[\right.$ pulse $\left.^{-1}\right]$ represents the outgassing rate. The overall change of the nitrogen concentration vs. the number of laser pulses $m$ is then:

$$
\frac{d c_{N}}{d m}=S_{\text {in }}-S_{\text {out }}=S_{\text {in }}-c_{N} / l
$$

and the solution of this differential equation is easily obtained:

$$
c_{N}(m)=S_{i n} l\left(1-e^{-m / l}\right) .
$$

The average nitrogen contents (neglecting the sharp surface peaks) vs. the number of laser pulses in 1 bar and 6 bar $\mathrm{N}_{2}$ are shown in Fig. 6.6 (symbols) with the corresponding fits according to Eq. (6.2) (lines). The value of the outgassing rate $1 / l$ is 0.0115 for the irradiation in 1 bar $\mathrm{N}_{2}$ and 0.010 for the irradiation in 6 bar $\mathrm{N}_{2}$, revealing its weak dependence on the ambient gas pressure, while the value of $S_{i n}$ increases with the nitrogen pressure. In order to better understand its dependence, we performed laser irradiations with 256 pulses at various pressures between 1 bar and 10 bar. The average nitrogen contents vs. the pressure are fitted according to Eq. (6.2) with fixed $m=256$ and using the mean value of $1 / l=0.0107(8)$. The results are shown in Fig. 6.7 the inset reports the RNRA nitrogen depth profiles at the different pressures. The increase of $S_{\text {in }}$ with the ambient pressure $p$ can be linearly fitted in the region 1-6 bar:

$$
S_{i n}=A+B p
$$

obtaining $A=0.087(7)$ [at.\% pulse ${ }^{-1}$ ] and $B=0.064(2)$ [at.\% pulse $^{-1}$ bar $^{-1}$. Using Eq. (6.2) and Eq. (6.3), the average nitrogen content in the aluminum matrix after excimer laser irradiation at $4 \mathrm{~J} / \mathrm{cm}^{2}$ can be estimated as a function of the ambient gas pressure $p$ and the number of laser pulses $m$ as follows:

$$
c_{N}(m, p)=\left(a_{1}+a_{2} p\right)\left(1-e^{-m / l}\right)
$$




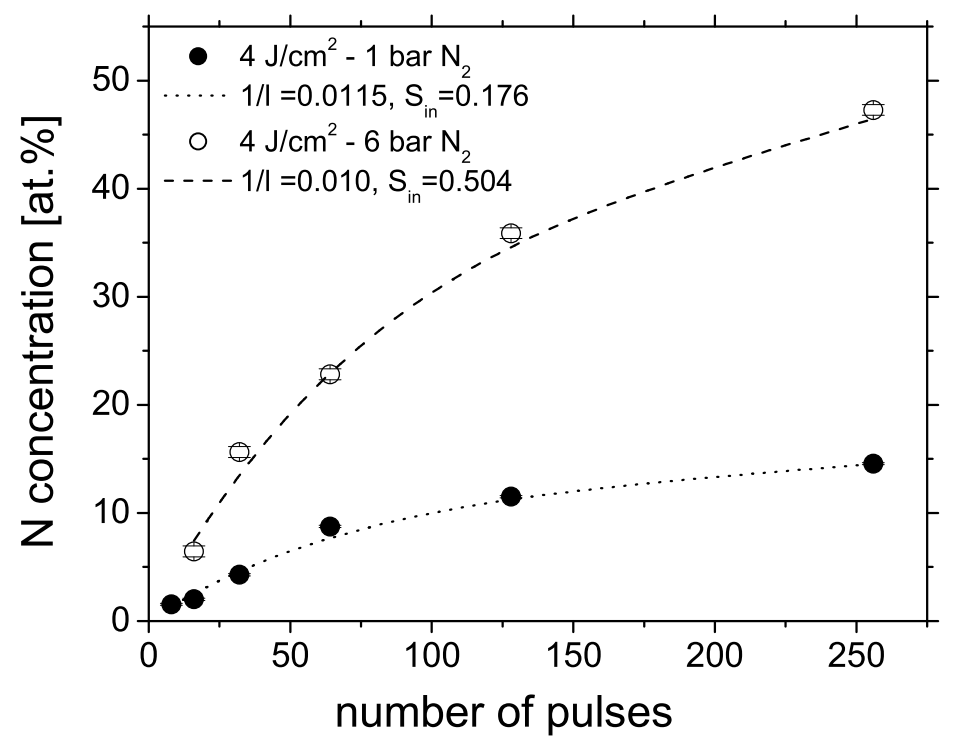

Figure 6.6: Average nitrogen content as a function of the number of laser pulses at $4 \mathrm{~J} / \mathrm{cm}^{2}$ in 1 bar $\mathrm{N}_{2}$ (solid symbols) and in 6 bar $\mathrm{N}_{2}$ (open symbols) with the corresponding fits according to Eq. (6.2).

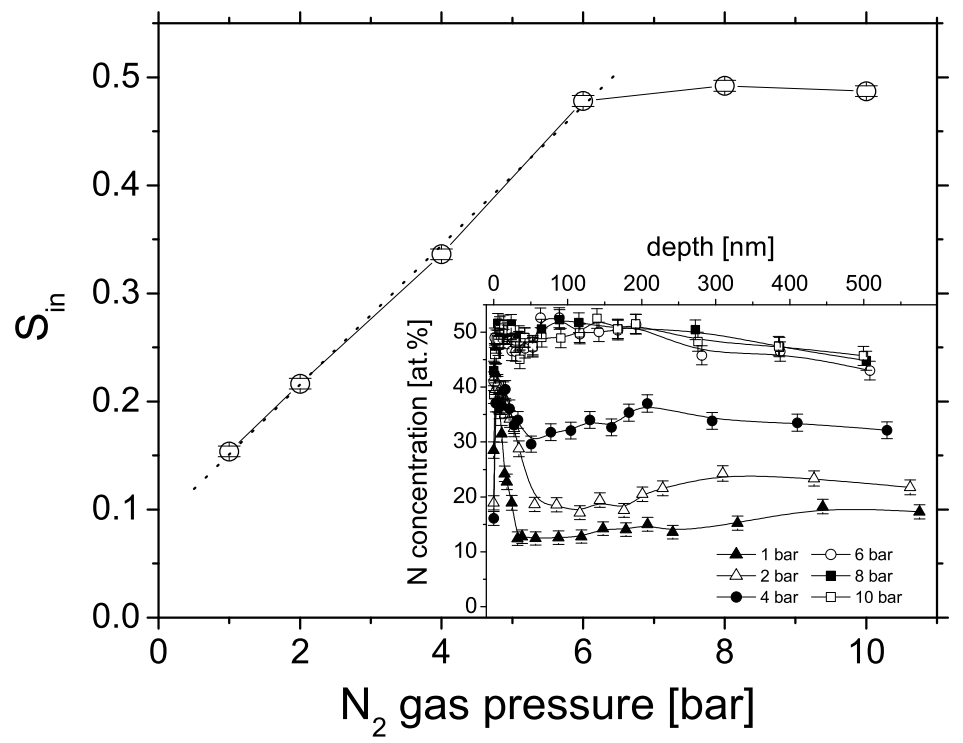

Figure 6.7: Experimental dependence of the parameter $S_{i n}$ on the ambient gas pressure. The dashed line is the linear fit in the region 1-6 bar. The inset depicts the corresponding nitrogen depth profiles used to extrapolate the values of $S_{i n}$.

with $a_{1}=A l=8.1(8)$ [at.\%] and $a_{2}=B l=6.0(4)$ [at.\% bar ${ }^{-1}$. The presented phenomenological model describes the take-up mechanism in a wide range of ambient 
pressures and number of laser shots. From the experimental evidence it can also be concluded that the melting time is long enough to homogenize the nitrogen distribution (unfortunately, no data about the diffusion of $\mathrm{N}$ in $\mathrm{Al}$ are available up to date).

\subsubsection{Mass transport mechanism at $\phi=2 \mathrm{~J} / \mathrm{cm}^{2}$}

The nitrogen depth profiles as a function of the number of laser pulses in the samples irradiated at $2 \mathrm{~J} / \mathrm{cm}^{2}$ in 1 bar $\mathrm{N}_{2}$ are shown in Fig. 6.8. Diffusion-like profiles with

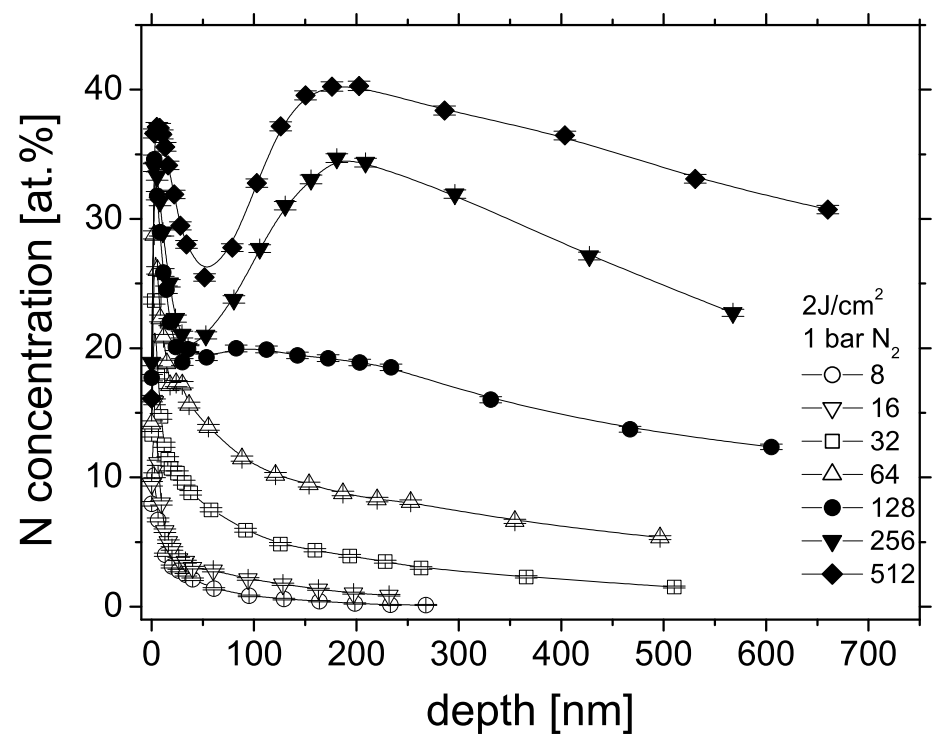

Figure 6.8: Nitrogen depth profiles after irradiation in 1 bar $\mathrm{N}_{2}$ at a fluence of $2 \mathrm{~J} / \mathrm{cm}^{2}$ and various numbers of laser pulses.

increasing nitrogen content and nitrided thickness are formed as the number of laser shots increases from 2 to 64 . A gradual modification of the profile shape is induced for a higher number of pulses, and the significant nitrogen content of $\sim 40$ at.\% is obtained. The simulation of the surface temperature and the evolution of the melting depth after irradiation of pure $\mathrm{Al}$ at $2 \mathrm{~J} / \mathrm{cm}^{2}$ are shown in Fig. 6.9. The melting time is about $230 \mathrm{~ns}$ and the maximum temperature reached at the surface is about $2000 \mathrm{~K}$, i.e. higher than the melting point of aluminum, but not high enough to dissociate or melt the AlN. This feature seems to determine the mass transport mechanism at this fluence. As verified by Transmission Electron Microscopy (TEM) on the samples irradiated 256 times at $2 \mathrm{~J} / \mathrm{cm}^{2}$ in 1 bar $\mathrm{N}_{2}$, small AlN particles embedded in the Al matrix are formed, with an average radius of the order of a few tens of nanometer (Fig. 6.10). The temperature reached in the sample during the laser irradiation is not sufficient to destroy these particles and they can move inside the liquid Al. In such a case, it is well established that the driving forces of the mass transport mechanism originate from the temperature and the chemical gradients [5] (gravitation-buoyancy plays a negligible role). For small particles floating inside a liquid, the chemical gradient is simply $\nabla n_{p}$, where $n_{p}$ is the number of particles 




Figure 6.9: Time evolution of the surface temperature of pure $\mathrm{Al}$ irradiated at $2 \mathrm{~J} / \mathrm{cm}^{2}$. The inset shows the melting depth vs. the melting time (the dashed curves represent the duration of the laser pulse).

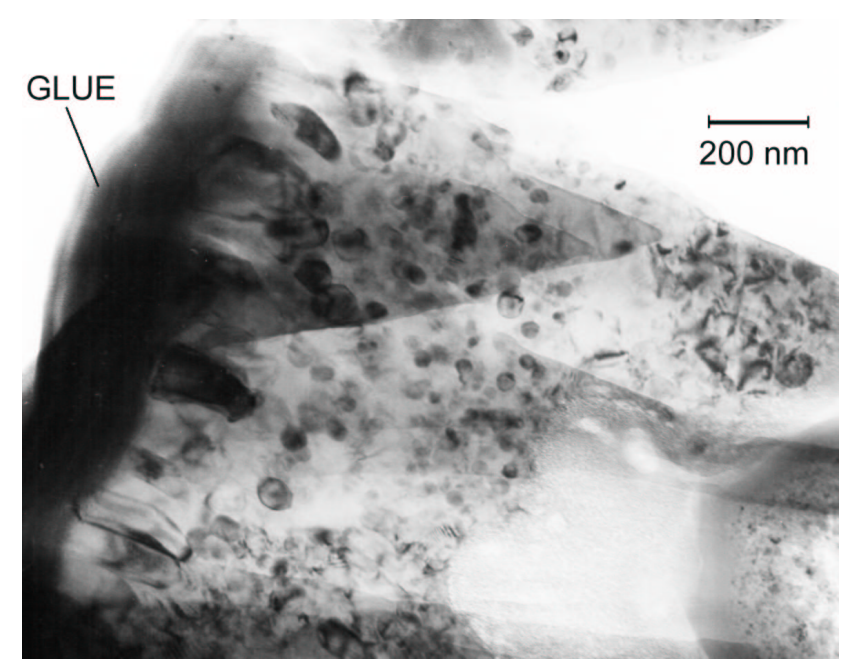

Figure 6.10: TEM picture of sample irradiated 256 times at $2 \mathrm{~J} / \mathrm{cm}^{2}$ in 1 bar $\mathrm{N}_{2}$. The small dark dots are the AlN grains.

per unit volume, and the diffusion mechanism is governed by the Brownian motion, with the diffusion constant $D_{b r}$ depending on the temperature $T$ of the fluid and on the radius $r$ of the solid particles [118, 119]:

$$
D_{b r}=\frac{k_{B} T}{6 \pi \eta r} .
$$

$\eta$ is the dynamic viscosity of the fluid and $k_{B}$ is the Boltzmann constant. At a temperature of about $2000 \mathrm{~K}$, with the viscosity of liquid Al being $10^{-3} \mathrm{~Pa} \cdot \mathrm{s}$ [120] and a particle radius 
of $10 \mathrm{~nm}$ we obtain $D_{b r} \sim 0.15 \mathrm{~nm}^{2} / \mathrm{ns}$. The particle flux $J_{b r}$ (i.e. the number of particles crossing the unit area per unit time) due to the Brownian motion is then:

$$
J_{b r}=-D_{b r} \frac{\partial n_{p}}{\partial z}
$$

The transport mechanism related to the temperature gradient is called thermophoresis [121, 122. It produces a drift of the floating particles in the direction opposite to the temperature gradient. The drift velocity for solid particles in a liquid has the following form [116]:

$$
v_{t h}=-\frac{2 \eta}{\rho T\left(2+\kappa_{i} / \kappa_{e}\right)} \frac{\partial T}{\partial z},
$$

where $\kappa_{i}$ and $\kappa_{e}$ are the thermal conductivities of the particle and the liquid, respectively. For a temperature gradient of $\sim 10^{9} \mathrm{~K} / \mathrm{m}$ (see Fig. 6.11) and using $\kappa_{e}=\kappa_{i}$ for simplicity, at $2000 \mathrm{~K}$ we have $v_{t h} \sim 0.12 \mathrm{~m} / \mathrm{s}$. The corresponding particle flux $J_{t h}$ is simply

$$
J_{t h}=v_{t h} n_{p}
$$

The total particles flux can be written as:

$$
J=J_{b r}+J_{t h}=-D_{b r} \frac{\partial n_{p}}{\partial z}+v_{t h} n_{p}
$$

and using the mass conservation law we obtain:

$$
\frac{\partial n_{p}}{\partial t}=-\nabla \cdot J=-\frac{\partial J}{\partial z}=\frac{\partial}{\partial z}\left(D_{b r} \frac{\partial n_{p}}{\partial z}-v_{t h} n_{p}\right)
$$

which is a version of the one-dimensional drift-diffusion equation [118]. It should be noticed that the time scale of the diffusion process and the thermophoretic drift might differ. In fact, the diffusion lasts as long as the Al matrix is molten, i.e. for a time of the order of the melting time $t_{m} \sim 230 \mathrm{~ns}$. The thermophoretic drift depends on the temperature gradient $\frac{\partial T}{\partial z}$. The simulation of $\frac{\partial T}{\partial z}$ in the first $600 \mathrm{~nm}$ from the surface of the sample (i.e. the mean depth investigated by RNRA) is plotted in Fig. 6.11 as a function of the time (the same computer code used to plot Figs. 6.5 and 6.9 has been used). The full width at half maximum is about $45 \mathrm{~ns}$, that represents the duration $t_{t h}$ of the thermophoretic drift. Eq. (6.10) was verified experimentally with some isotopic irradiations. Similarly to the notation used in section 5.1, the term $m^{*} / k$ refers to $m$ pulses in enriched ${ }^{15} \mathrm{~N}$ gas followed by $k$ pulses in natural nitrogen. Fig. 6.12 reports the nitrogen depth profiles (symbols) of the $256^{*} / 0,256^{*} / 4,256^{*} / 8$ and $256^{*} / 16$ irradiations. Eq. (6.10) was written according to the finite difference method and the numerical fit of the $256^{*} / 0$ nitrogen depth profile was used as the initial condition. Since the number of particles per unit volume $n_{p}$ is proportional to the nitrogen concentration $c_{N}$, the substitution of $n_{p}$ by $c_{N}$ in Eq. 6.10) is harmless. The surface boundary condition

$$
\left.\frac{\partial c_{N}}{\partial z}\right|_{z=0}=0
$$

was used to exclude any material flux through the surface of the sample. The evolution of the 256*/0 profile after 4, 8 and 16 laser pulses according to Eq. 6.10) is plotted 




Figure 6.11: Time evolution of the temperature gradient averaged over $600 \mathrm{~nm}$ from the surface of the $\mathrm{Al}$ sample irradiated at $2 \mathrm{~J} / \mathrm{cm}^{2}$.

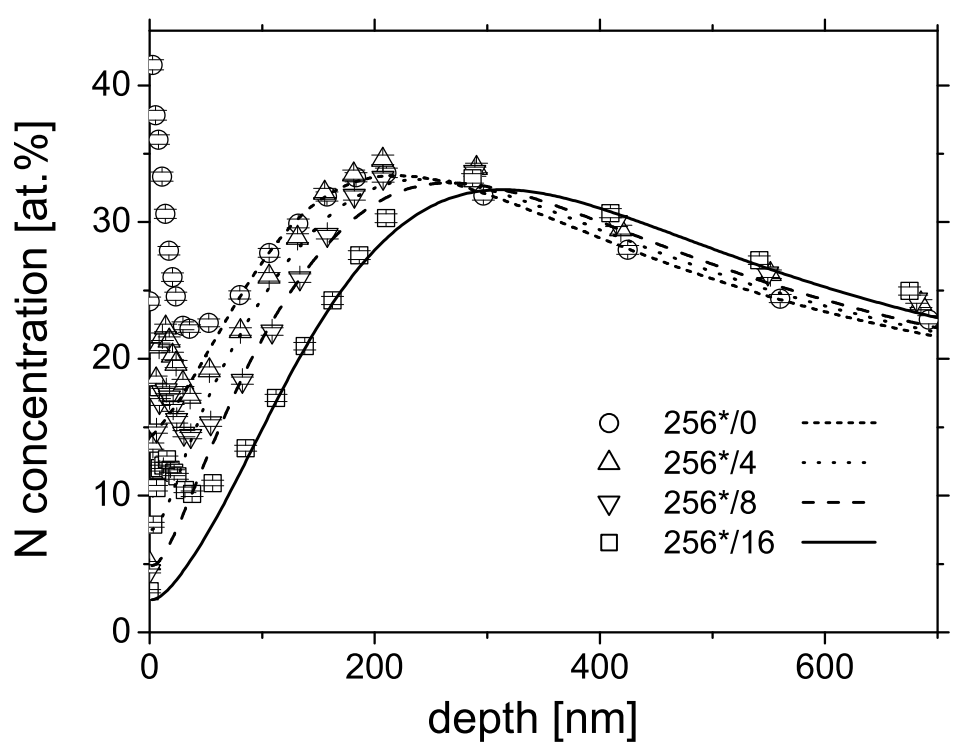

Figure 6.12: Nitrogen depth profiles of the isotopic experiments $256^{*} / 0$ (०), $256^{*} / 4(\triangle), 256^{*} / 8$ $(\nabla), 256^{*} / 16(\square)$ at $2 \mathrm{~J} / \mathrm{cm}^{2}$ in 1 bar $\mathrm{N}_{2}$ with the corresponding simulations according to Eq. 6.10.

(lines) in Fig. 6.12, showing excellent agreement with the experimental data. The values of $v_{t h}=0.10 \mathrm{~nm} / \mathrm{ns}, t_{t h}=45 \mathrm{~ns}, D_{b r}=0.5 \mathrm{~nm}^{2} / \mathrm{ns}$ and $t_{m}=230 \mathrm{~ns}$ were used in the simulations as fitting parameters. The values of $v_{t h}$ and $t_{t h}$ are consistent with the 
results obtained from the thermal simulations. The diffusion constant $D_{b r}=0.5 \mathrm{~nm}^{2} / \mathrm{ns}$ corresponds to a particle radius of $\sim 2 \mathrm{~nm}$, which is somewhat smaller than the average dimension observed with TEM. However, the analytical technique used to measure the nitrogen depth profiles involves the use of a charged ion beam that is subject to straggling. According to the model of Lindhard and Scharff (Eq. (4.14) and Fig. 4.8), the straggling of a $430 \mathrm{keV}$ proton beam in aluminum at a depth of $170 \mathrm{~nm}$ is of the order of $21 \mathrm{~nm}$. This value corresponds to an apparent diffusion length of $\sim \sqrt{D_{a p p} t_{m}}$. If $t_{m}=230 \mathrm{~ns}$, we obtain $D_{a p p}=1.9 \mathrm{~nm}^{2} / \mathrm{ns}$. Due to the thermophoretic drift, after 16 laser pulses the nitrogen profile moves to $170+16 v_{t h} t_{t h} \simeq 240 \mathrm{~nm}$ and the straggling becomes about $25 \mathrm{~nm}$, corresponding to an apparent diffusion coefficient $D_{a p p}=2.7 \mathrm{~nm}^{2} / \mathrm{ns}$. Therefore, the RNRA depth resolution, especially at large depths, can be inadequate to measure small diffusion constants. This may explain the discrepancy between the measured and the predicted value of $D_{b r}$. However, the beam straggling is smaller than the overall thermophoretic drift, even after 4 pulses, and this effect can be clearly distinguished. The entire mass transport process at $2 \mathrm{~J} / \mathrm{cm}^{2}$ is schematically summarized in Fig. 6.13 and the main thermophysical parameters of the Al-AlN system are reported in Table 6.1. The modeling of the nitrogen depth profiles as a function of the number of laser pulses requires the detailed knowledge of the nitrogen incorporation mechanism, that might depend on the properties of the surface (roughness, reflectivity, nitrogen content, etc.) and therefore on the number of pulses itself, with a high degree of complexity.

Table 6.1: List of the main thermal and physical parameters of $\mathrm{Al}$ and $\mathrm{AlN}$.

\begin{tabular}{lrrr}
\hline \hline Parameter & Symbol & Value & Ref. \\
\hline Al melting temperature & $\mathrm{T}_{m}(\mathrm{Al})$ & $933.5 \mathrm{~K}$ & {$[43]$} \\
Al boiling temperature & $\mathrm{T}_{b}$ & $2740 \mathrm{~K}$ & {$[43]$} \\
Al latent heat of fusion & $\mathrm{L}_{m}$ & $10.5 \mathrm{~kJ} / \mathrm{mole}$ & {$[43]$} \\
Al latent heat of evaporation & $\mathrm{L}_{e v}$ & $296 \mathrm{~kJ} / \mathrm{mole}$ & {$[43]$} \\
Al reflectivity (at $\lambda=308 \mathrm{~nm})$ & $\mathrm{R}_{0}$ & $\simeq 0.5$ & $(a)$ \\
Al absorption length $($ at $\lambda=308 \mathrm{~nm})$ & $1 / \alpha$ & $7 \mathrm{~nm}$ & {$[43]$} \\
Al molar mass & $\mathrm{M}$ & $27 \mathrm{~g}$ & {$[43]$} \\
Al density & $\rho_{A l}$ & $2.7 \mathrm{~g} / \mathrm{cm}^{3}$ & {$[43]$} \\
liquid Al viscosity & $\eta$ & $10^{-3} \mathrm{~Pa} \mathrm{~s}_{0}$ & {$[120]$} \\
AlN melting temperature & $\mathrm{T}_{m}(\mathrm{AlN})$ & $3070 \mathrm{~K}$ & {$[38]$} \\
AlN dissociation temperature & $\mathrm{T}_{d}$ & $2400 \mathrm{~K}$ & {$[48]$} \\
AlN density & $\rho_{A l N}$ & $3.25 \mathrm{~g} / \mathrm{cm}^{3}$ & {$[43]$} \\
\hline \hline
\end{tabular}

(a) Measured.

\subsubsection{Crystal structures and mechanical properties}

The crystalline structure of the nitrided layers was investigated in the samples irradiated at $2 \mathrm{~J} / \mathrm{cm}^{2}$ and $4 \mathrm{~J} / \mathrm{cm}^{2}$ in 1 bar $\mathrm{N}_{2}$ with 256 pulses. The diffractograms reported in Fig. 6.14 show the formation of polycrystalline AlN with the wurtzite structure, as 

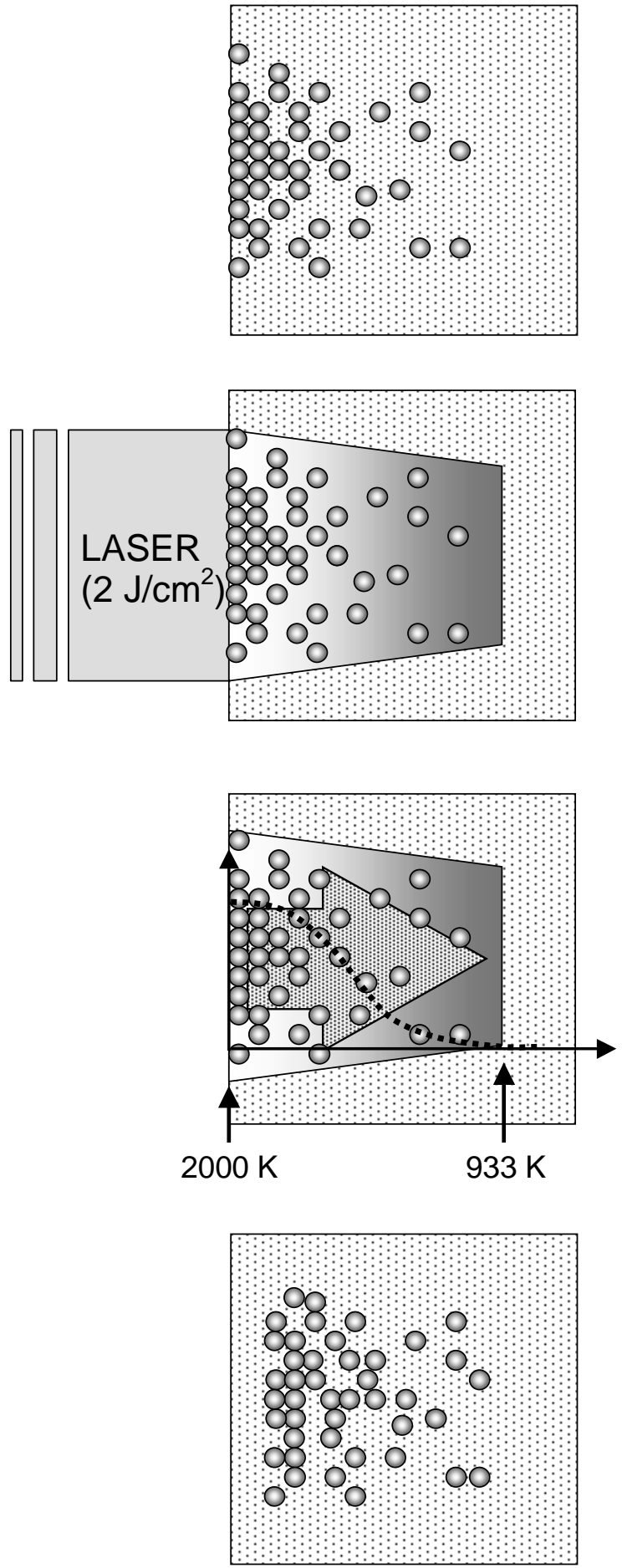

1) INITIAL CONDITION (after 256 laser shots in $\mathrm{N}_{2}$ ): SMALL GRAINS OF AIN ARE EMBEDDED IN THE AL MATRIX.

2) LASER IRRADIATION: THE AI MATRIX IS MOLTEN, BUT THE AIN GRAINS ARE NOT DISSOLVED.
4) SOLIDIFICATION: ALL AIN GRAINS MOVED TO LARGER DEPTH IN THE AI MATRIX.

Figure 6.13: Schematic view of the mass transport mechanism of $\mathrm{AlN}$ in the $\mathrm{Al}$ matrix during the irradiation at a laser fluence of $2 \mathrm{~J} / \mathrm{cm}^{2}$.

revealed by the Rietveld refinements. The lattice parameters of the Al matrix and the AlN phase reported in Table 6.2 are in good agreement with the bulk values [44]. No evidence of oxides, NaCl-type or zincblende-type AlN was found (the latter is considered a nonequilibrium crystal structure: it was observed for $\mathrm{GaN}$ and $\mathrm{InN}$, but only theoretically 


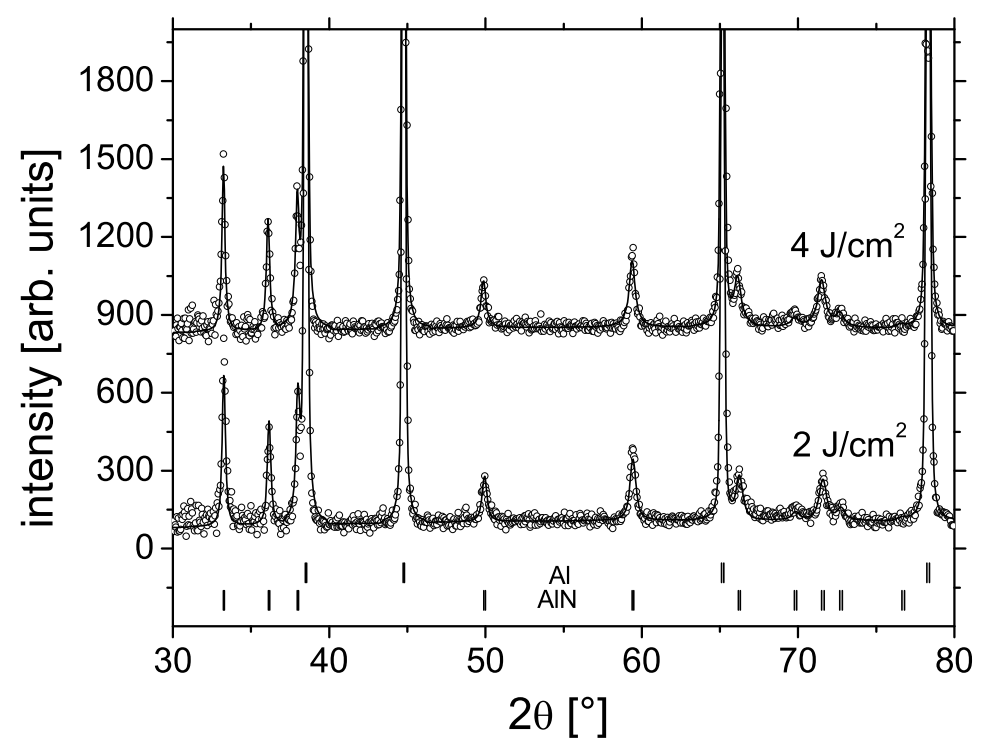

Figure 6.14: $1.5^{\circ}$ GIXRD diffractograms of the Al sample irradiated at $2 \mathrm{~J} / \mathrm{cm}^{2}$ and $4 \mathrm{~J} / \mathrm{cm}^{2}$, with 256 pulses in 1 bar $\mathrm{N}_{2}$. The continuous lines are the Rietveld refined fits and the small vertical bars correspond to the Bragg positions of the various crystallographic directions.

predicted for AlN [51, 123]). The mechanical properties of the irradiated samples have

Table 6.2: Lattice parameters obtained from the Rietveld refinements shown in Fig. 6.14. The parameter " $u$ " is the fractional coordinate along the c axis of the AlN cell that determines the position of the nitrogen atoms in the wurtzite structure.



been investigated by nanoindentation. The values of the hardness reported in Fig 6.15 have been taken at the indentation depth of $100 \mathrm{~nm}$ for all profiles in order to compare the results consistently. A large increase in the surface hardness for increasing number of laser shots and nitrogen pressure has been observed in all the nitrided specimens, regardless of the laser fluence (only in the sample irradiated at $4 \mathrm{~J} / \mathrm{cm}^{2}$ in 10 bar nitrogen we noticed a decrease attributed to the surface roughness). The highest value of $5 \mathrm{GPa}$, i.e 10 times larger than the untreated aluminum substrate, has been observed in the sample irradiated at $4 \mathrm{~J} / \mathrm{cm}^{2}$ in 8 bar $\mathrm{N}_{2}$. The correlation between the nitrogen content and the surface hardness is evidenced in Fig. 6.16 as function of the gas pressure, revealing how the mechanical properties are closely connected to the formation of the nitride. A similar feature was observed in the iron substrates irradiated in nitrogen [31. 




Figure 6.15: Hardness of the $\mathrm{Al}$ samples nitrided at $2 \mathrm{~J} / \mathrm{cm}^{2}(\circ)$ and $4 \mathrm{~J} / \mathrm{cm}^{2}(\bullet)$ as a function of the number of laser shots (left) and the nitrogen pressure (right).

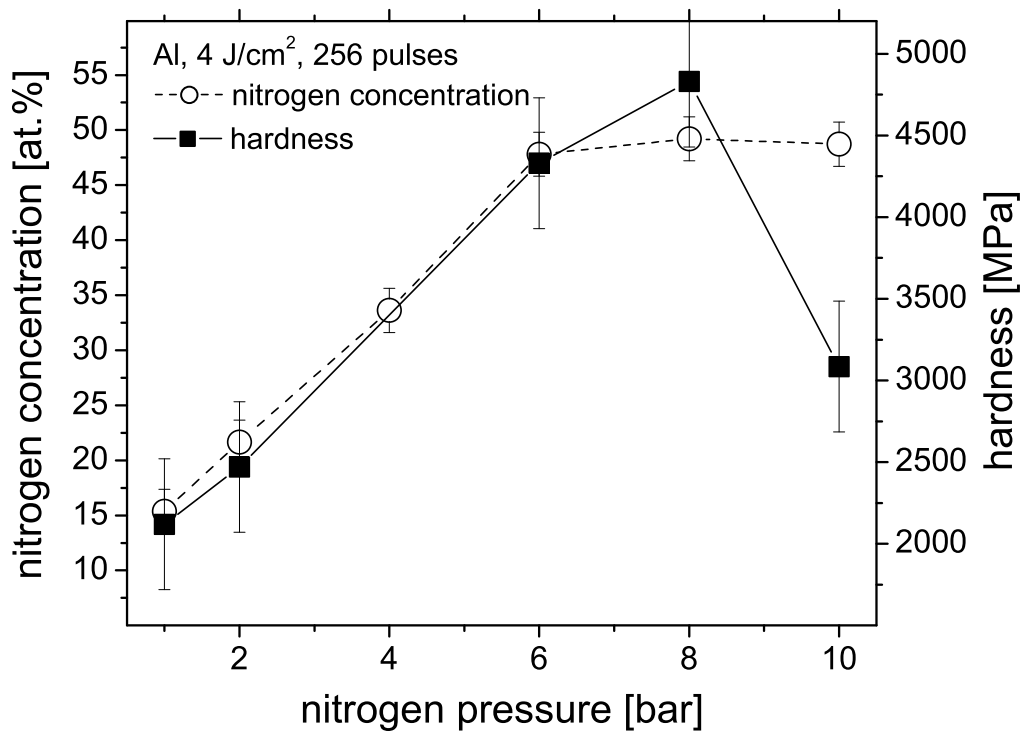

Figure 6.16: Correlation between the surface hardness and the average nitrogen content in the samples irradiated with 256 pulses at $4 \mathrm{~J} / \mathrm{cm}^{2}$.

\subsection{Laser irradiation in methane atmosphere}

Aluminum carbide $\left(\mathrm{Al}_{4} \mathrm{C}_{3}\right)$ does not meet the technological "fortune" of aluminum nitride. Few publications and little attention has been given to this compound in comparison to its nitride counterpart. Nevertheless the laser irradiation of aluminum substrates in methane 
atmosphere has been performed in order to demonstrate that the carbon incorporation is effective. The sample treated with the $16 \times 16$ meander scan at the laser fluence of

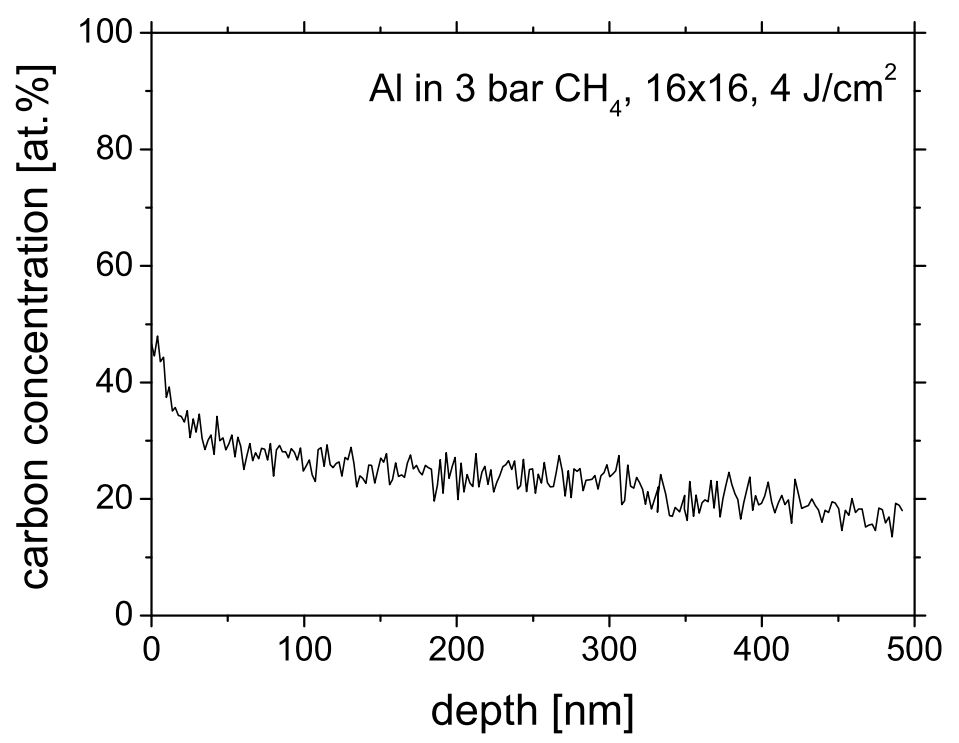

Figure 6.17: RBS carbon depth profile of the aluminum sample meandered in 3 bar $\mathrm{CH}_{4}$.

$4 \mathrm{~J} / \mathrm{cm}^{2}$ in 3 bar $\mathrm{CH}_{4}$ has been analyzed by means of RBS, XRD and nanoindentation. The RBS measurement revealed an average carbon content of about 20 at.\%, as shown in Fig. 6.17. The nanoindentation measurement showed a considerable increase of the

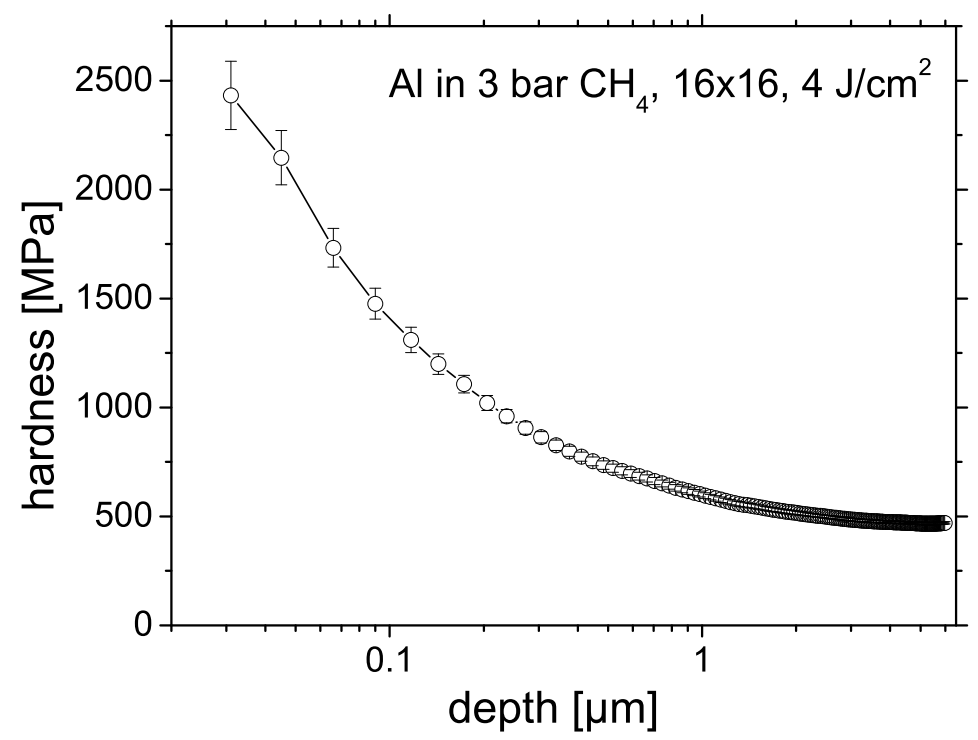

Figure 6.18: Hardness profile of the laser carburized Al substrate. 
surface hardness after the treatment (about 4 times larger than the virgin substrate) as reported in the Fig. 6.18. Nevertheless, very poor crystallinity has been observed by GIXRD (with incidence angle of $1^{\circ}$ ): a broad peak centered at the Bragg angle $2 \theta=31.6^{\circ}$ $\left(7.2^{\circ} \mathrm{FWHM}\right)$ appears in the diffraction patter of the irradiated specimen, as reported in Fig. 6.19. The position of the Bragg reflection is in good agreement with the value of the most intense diffraction peak of crystalline $\mathrm{Al}_{4} \mathrm{C}_{3}\left(<012>\right.$ peak at $\left.2 \theta=31.74^{\circ}\right)$, suggesting the formation of amorphous aluminum carbide. The poor crystallinity is attributed to the more complex atomic arrangement of the the $\mathrm{Al}_{4} \mathrm{C}_{3}$ phase compared to $\mathrm{AlN}$. The rapid quenching rate (see Fig. 6.11) obtained with nanosecond laser pulses might hinder the long-range atomic order. Thus, even if the basic $\mathrm{Al}_{4} \mathrm{C}_{3}$ arrangement is locally formed, the crystallites are not large enough to give a clear XRD signal, and only the main interatomic distance is observed (i.e. the most intense XRD peak). Similar effects have been found in the silicon substrates irradiated in nitrogen atmosphere (see section 7.2), where the stoichiometry of the nitride phase is $\mathrm{Si}_{3} \mathrm{~N}_{4}$ (the role of $\mathrm{Al}$ atom in the aluminum carbide is played by the $\mathrm{N}$ atom is the silicon nitride).

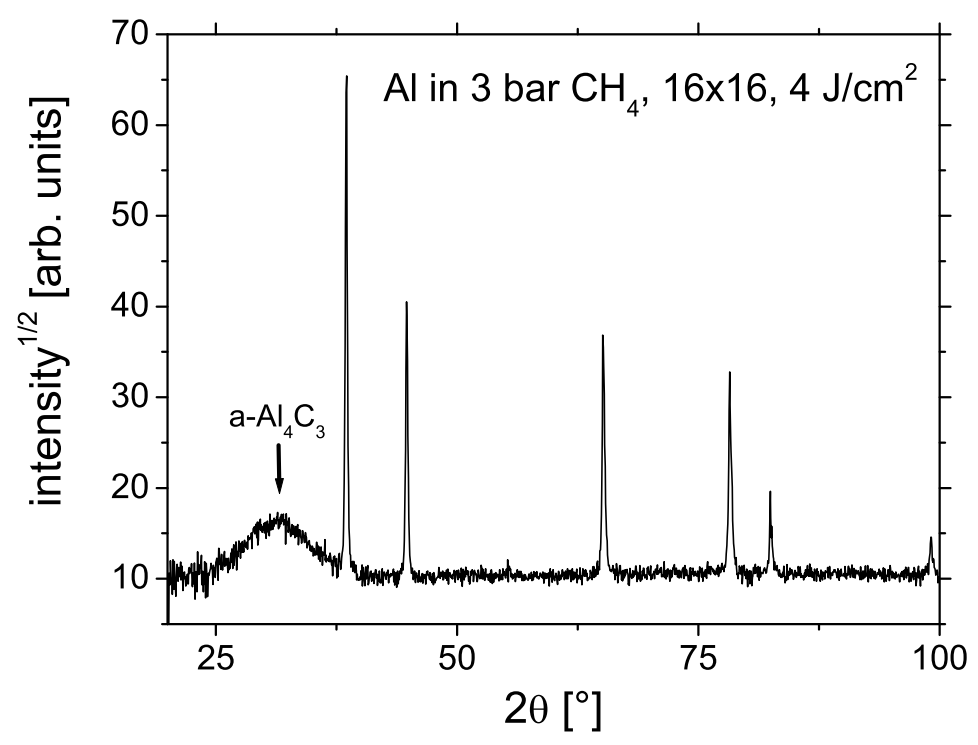

Figure 6.19: $1^{\circ}$ GIXRD patter of the laser carburized sample. The broad peak at $2 \theta=31.6^{\circ}$ indicates the formation of amorphous $\mathrm{Al}_{4} \mathrm{C}_{3}$, all other peak belong to the fcc $\mathrm{Al}$ matrix. 


\section{Chapter 7}

\section{The silicon substrate}

The following sections will show how the laser irradiation of single-crystalline silicon substrates in controlled nitrogen and methane atmospheres leads to the formation of carbide and nitride phases [124]. The analysis has been performed mainly (but not only) by Extended X-ray Absorption Fine Structure (EXAFS) due to the high sensitivity of this technique to the local atomic environment. As already described in section 4.5, the EXAFS information is extracted from the wiggles of the X-ray absorption coefficient above the absorption edge. Fig. 7.1 shows the fine structure oscillations (weighted with factor $k^{2}$ ) of the virgin silicon substrate, commercial $\beta$-SiC powder, commercial $\alpha-\mathrm{Si}_{3} \mathrm{~N}_{4}$ powder and the silicon samples irradiated with 256 laser pulses in $\mathrm{CH}_{4}$ and in $\mathrm{N}_{2}$.

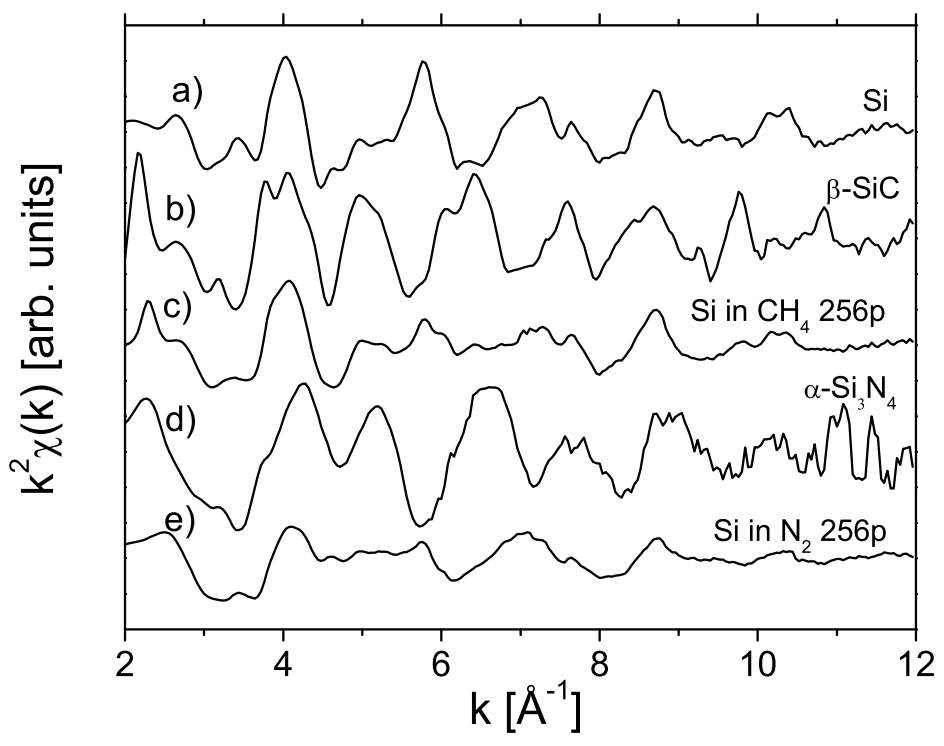

Figure 7.1: Fine structure oscillations weighted with the $k^{2}$ factor of: a) $\mathrm{Si}<100>$, b) $\beta$-SiC, c) Si irradiated in $\mathrm{CH}_{4}$ with 256 pulses, d) $\alpha$ - $\mathrm{Si}_{3} \mathrm{~N}_{4}$, e) Si irradiated in $\mathrm{N}_{2}$ with 256 pulses. 


\subsection{Laser irradiation in methane atmosphere}

Aiming to the formation of $\mathrm{SiC}$, the influence of the number of laser pulses on the carbon incorporation into the silicon matrix has been investigated with the single spot treatment, using the raw laser beam (the homogenizer lens barely allows to reach the fluence of $4 \mathrm{~J} / \mathrm{cm}^{2}$, which is the threshold fluence to induce strong vaporization in the silicon substrate). As it will be shown, the synthesis of $\mathrm{SiC}$ is successful, but its optimization requires the investigation of other experimental parameters: thus, the effect of the meandering scan combined with the methane gas pressure will be described in section 7.1.2.

\subsubsection{Influence of the number of pulses}

The single spot laser treatments were performed at fixed laser fluence of $5 \mathrm{~J} / \mathrm{cm}^{2}$ in 2 bar $\mathrm{CH}_{4}$, varying the number of laser shots between 2 and 256. The EXAFS oscillations of the laser-carburized samples were Fourier-transformed in the region $2 \AA^{-1} \leq k \leq 12 \AA^{-1}$. The resulting curve of the Si sample irradiated with 256 pulses is reported in Fig. 7.2 , For comparison, the Fourier transform (FT) of the virgin silicon substrate and the $\beta$-SiC standard are reported as well. It is clear at a first glance that the laser-irradiated specimen

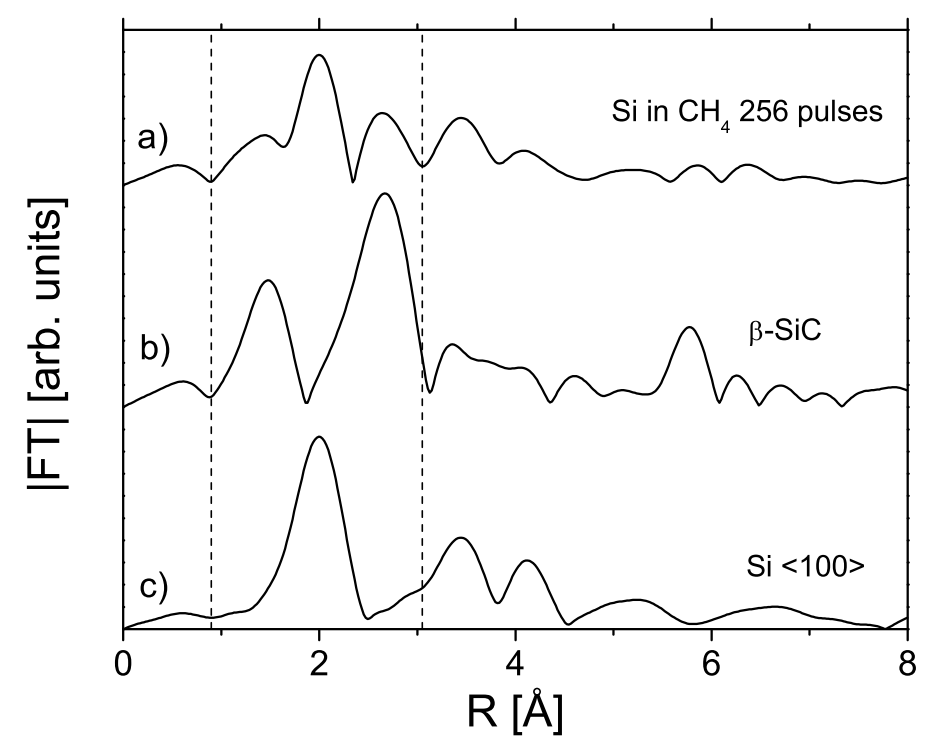

Figure 7.2: Fourier transform moduli of: a) Si sample irradiated in $\mathrm{CH}_{4}$ with 256 pulses, b) $\beta$-SiC standard, c) virgin $\mathrm{Si}<100>$. The dashed vertical lines delimit the BFT region.

is a mixture of the carbide phase and the non-reacted $\mathrm{Si}$, since peaks belonging to both structures are superimposed. In order to have quantitative information, the back Fourier transform (BFT) analysis based on a three-shells fit has been carried out. The BFT was calculated in the region $0.90 \AA<R<3.05 \AA$ including the peaks corresponding to the first shell of $\mathrm{SiC}$ (C scatterer, peak at $\sim 1.5 \AA$ ), the first shell of $\mathrm{Si}$ (peak at $\sim 2.05 \AA$ ) and the second shell of $\mathrm{SiC}$ ( $\mathrm{Si}$ scatterer, peak at $\sim 2.6 \AA$ ). The scattering amplitudes 
and the phases of each shell were obtained from the standard specimens (pure Si and $\beta$-SiC) and loaded in the fits as initial parameters. Since the absorption edges of $\mathrm{Si}$ and $\mathrm{SiC}$ differ by $2 \mathrm{eV}$ (1839 eV and $1841 \mathrm{eV}$, respectively), this energy correction has been introduced as a fixed parameter in the fitting procedure of the first and second shell of $\mathrm{SiC}$. The oscillations extrapolated from the BFT of the silicon sample irradiated with 256 laser shots are shown in Fig. 7.3. They have been fitted according to Eq. (4.46) in

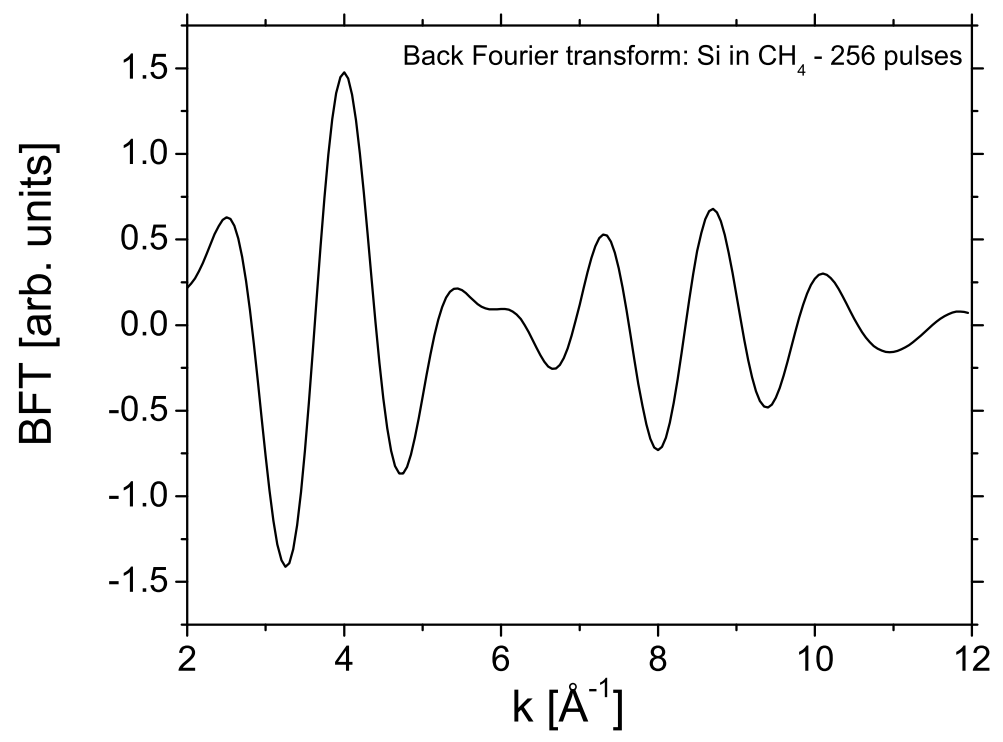

Figure 7.3: Back Fourier transform oscillations of the Si sample irradiated with 256 laser pulses in 2 bar $\mathrm{CH}_{4}$.

order to obtain the radii, the occupancies and the disorder factors of the three shells. The disorder parameters $\Delta \sigma^{2}$, relative to the standards, were found to be less than $4 \times 10^{-4} \AA^{2}$, confirming the good crystallinity of the laser-induced phases. To evaluate the $\mathrm{Si}$ to $\mathrm{SiC}$ phase ratio, the numbers of neighbors obtained from the analysis have been normalized with respect to the ideal values of the pure substances. There are four $\mathrm{C}$ neighbors at the distance of $1.89 \AA$ in the first shell of $\mathrm{SiC}$ (in tetrahedral arrangements), four Si neighbors at the distance of $2.35 \AA$ in the first shell of $\mathrm{Si}$ (in tetrahedral arrangements) and $12 \mathrm{Si}$ neighbors at the distance of $3.08 \AA$ in the second shell of $\mathrm{SiC}$ (in fcc configuration). The results of the fits are reported in Fig. 7.4 and Table 7.1. The radii of the shells are in good agreement with the predicted values, and the relative amounts of SiC calculated independently from each shell are very close, indicating the good morphology of the basic carbide structure. The fraction $f_{S i}$ of non-reacted Si has been related to the fraction $f_{S i C}$ of SiC simply as $f_{S i C}=1-f_{S i}$. To cross check the results of the fitting, we compared the weighted oscillations $k^{2} \chi(k)$ of the laser treated samples with the linear combination of the weighted oscillations of $\mathrm{Si}$ and $\beta$-SiC in the entire region $2 \AA^{-1} \leq k \leq 12 \AA^{-1}$. The result reported in Fig. 7.4 (solid symbol) shows an excellent agreement with the shells fitting, confirming once more the good degree of crystallinity of the laser-induced $\mathrm{SiC}$. We point out that the relative amount of carbide phase seems to saturate at about $35 \%$ after 256 laser pulses and a higher number of laser shots would hardly lead to any 


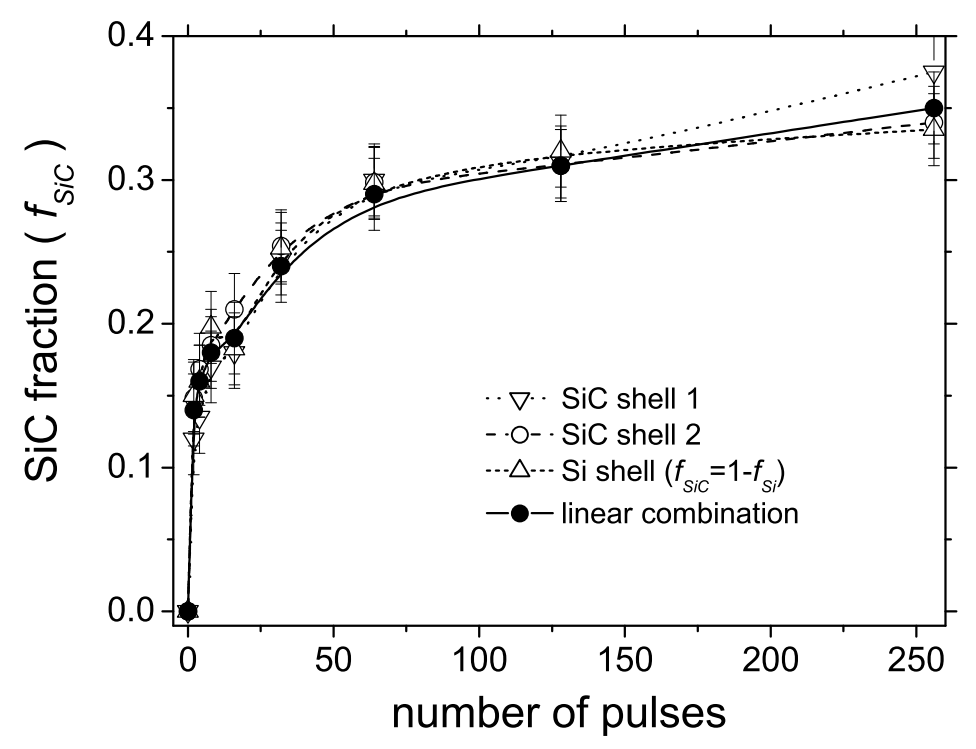

Figure 7.4: $\mathrm{SiC}$ phase fraction obtained from the EXAFS analysis as a function of the number of laser pulses: $(\nabla)$ first shell of $\mathrm{SiC},(\circ)$ second shell of $\mathrm{SiC},(\triangle)$ first shell of $\mathrm{Si},(\bullet)$ linear combination (see text).

Table 7.1: Three-shells fitting of the Si samples irradiated in $\mathrm{CH}_{4}$ : summary of the results.

\begin{tabular}{ccccccc}
\hline \hline Laser shots & \multicolumn{2}{c}{ Si-C (I shell) } & \multicolumn{2}{c}{ Si (I shell) } & \multicolumn{2}{c}{ Si-C (II shell) } \\
& $\mathrm{N} / 4$ & $\mathrm{R}[\AA]$ & $\mathrm{N} / 4$ & $\mathrm{R}[\AA]$ & $\mathrm{N} / 12$ & $\mathrm{R}[\AA]$ \\
\hline 2 & 0.120 & 1.90 & 0.850 & 2.35 & 0.148 & 3.11 \\
4 & 0.135 & 1.88 & 0.840 & 2.35 & 0.168 & 3.11 \\
8 & 0.170 & 1.89 & 0.802 & 2.35 & 0.185 & 3.09 \\
16 & 0.180 & 1.89 & 0.818 & 2.35 & 0.210 & 3.09 \\
32 & 0.245 & 1.89 & 0.748 & 2.35 & 0.254 & 3.08 \\
64 & 0.300 & 1.89 & 0.703 & 2.35 & 0.298 & 3.08 \\
128 & 0.313 & 1.89 & 0.680 & 2.35 & 0.310 & 3.07 \\
256 & 0.375 & 1.86 & 0.665 & 2.35 & 0.340 & 3.07 \\
\hline \hline
\end{tabular}

increase in the amount of $\mathrm{SiC}$ under the actual experimental conditions. Silicon carbide crystallizes in many polytypes differing from each other only in the stacking sequence. The two most common $\mathrm{SiC}$ polytypes are the $3 \mathrm{C}-\mathrm{SiC}$ with cubic structure, and the $2 \mathrm{H}-\mathrm{SiC}$ with hexagonal structure (see section 2.3). The EXAFS oscillations are sensitive to the short range atomic order and all types have the same fine structure pattern. GIXRD was employed to have information on the crystallographic phase of the laser-induced $\mathrm{SiC}$ and the diffractogram of the sample irradiated 256 times in $\mathrm{CH}_{4}$ is reported in Fig. 7.5, showing the formation of polycrystalline 3C-SiC $(\beta$-SiC). In glancing incidence geometry, the peak corresponding to the orientation of the single crystal is not observable. Nevertheless, the laser melting leads to the formation of a polycrystalline Si layer and therefore all Si 
peaks become visible. The EXAFS analysis revealed also the influence of the meandering

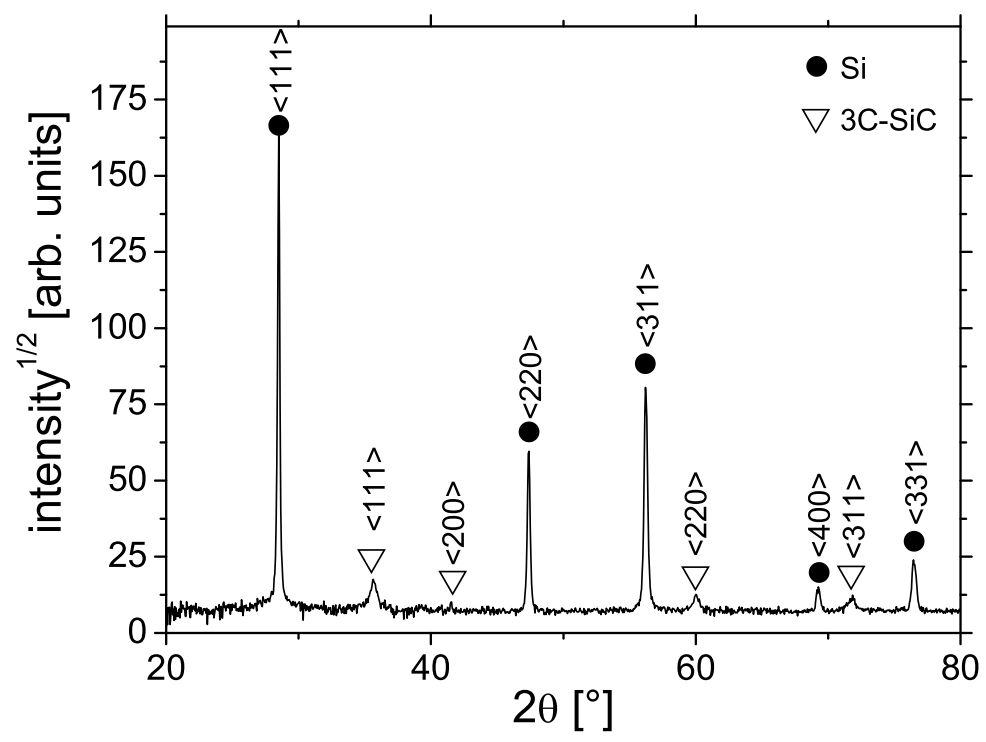

Figure 7.5: $2^{\circ}$ GIXRD diffractogram of the Si sample irradiated in $\mathrm{CH}_{4}$ with 256 pulses: $(\nabla)$ cubic $\mathrm{SiC},(\bullet)$ polycrystalline $\mathrm{Si}$.

treatment on the transport mechanism of carbon. As reported in Fig. 7.6, the intensities of the carbide peaks in the sample meandered with the $16 \times 16$ spot overlap are higher compared to the intensities in the sample treated under identical conditions but as single spot with 256 laser pulses. The carbon depth profiles extracted from the RBS analysis of these two samples are reported in the inset of Fig. 7.6, showing that the carbon contents on the surface are consistent with the EXAFS observations and indicating that the single spot treatment leads to more homogeneous concentration profiles compared to the meandering scan. Nevertheless, to maximize the carbon content (and therefore the amount of $\mathrm{SiC}$ ) the meander treatment seems to be more appropriate.

\subsubsection{Influence of the methane pressure}

To investigate the influence of the methane pressure on the carbon incorporation, $12 \times 12$ meander scans at the fixed laser fluence of $5 \mathrm{~J} / \mathrm{cm}^{2}$ in $0.5,1$ and 4 bar $\mathrm{CH}_{4}$ have been performed. The FT of the corresponding EXAFS oscillations are reported in Fig. 7.7. The intensity of the Si peak increases with increasing ambient pressure, indicating that the relative amount of carbide is higher for lower methane pressure. A similar effect was observed also in the iron samples irradiated in $\mathrm{CH}_{4}$, where a layer of amorphous carbon was formed at low methane pressure on the target surface (see Fig. 5.13). As already pointed out in section 5.2 .2 this phenomenon might be correlated to the shielding effect of the ambient gas on the laser beam. The quantitative analysis of the EXAFS data has been performed fitting the back Fourier transform (BFT) of the first three shells, similarly to the procedure described in the previous section. The average $\mathrm{SiC}$ relative fraction vs. 


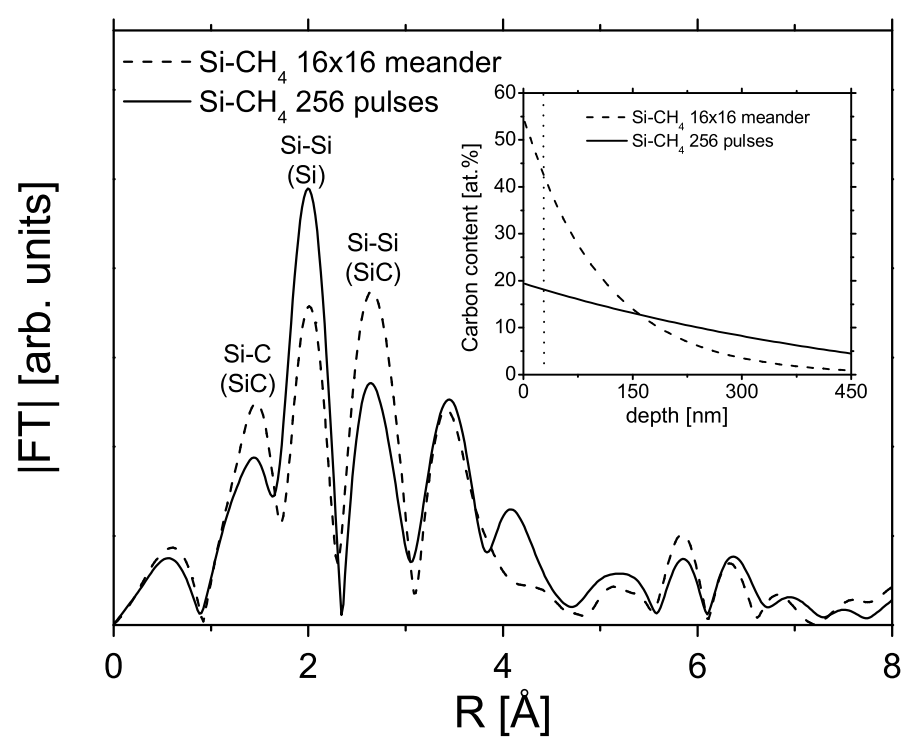

Figure 7.6: Fourier transform moduli of the samples irradiated in $\mathrm{CH}_{4}$ : single spot with 256 pulses (solid line) and $16 \times 16$ meander scan (dashed line). The inset show the corresponding carbon depth profiles measured by RBS. The vertical dotted line is the average information depth of the EXAFS in TEY mode $(\sim 30 \mathrm{~nm})$.

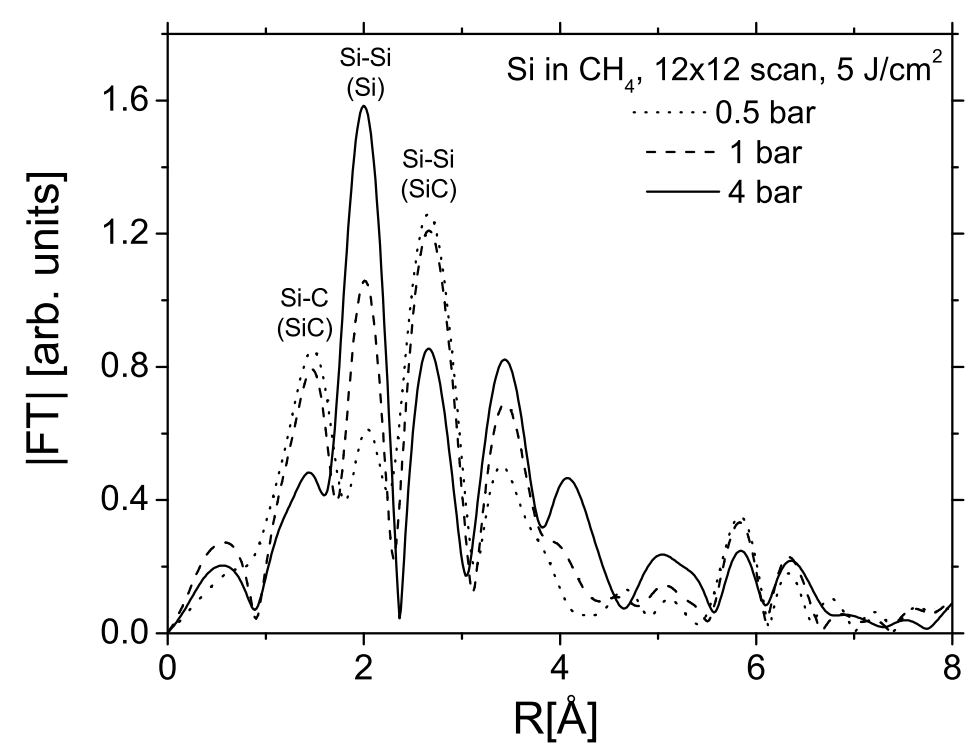

Figure 7.7: Fourier transform moduli of the Si samples meandered in 0.5 bar (dotted line), 1 bar (dashed line) and 4 bar (solid line) $\mathrm{CH}_{4}$.

the methane pressure is reported in Fig. 7.8. At 0.5 bar $\mathrm{CH}_{4}$ the carbide fraction is about $65 \%$, decreasing to less than $30 \%$ at 4 bar. Besides, the carbon depth profiles measured 




Figure 7.8: $\mathrm{SiC}$ phase fraction vs. the methane gas pressure as obtained from the EXAFS analysis. The inset reports the corresponding carbon depth profiles.

by RBS (see inset in Fig. 7.8) revealed that at the lowest methane pressure, the most effective carbon incorporation is achieved. Even if a pure and homogeneous SiC layer is not obtained, these results suggest that the optimal conditions to form $\mathrm{SiC}$ by irradiating $\mathrm{Si}$ in methane gas are: (i) a number of pulses/spot overlap not lower than 100 and (ii) an ambient pressure not higher than 0.5 bar.

\subsection{Laser irradiation in nitrogen atmosphere}

In the next sections, the results of the silicon irradiation in nitrogen atmosphere will be reported. Special emphasis will be given to the mass transport mechanisms during the single spot and the meandering treatments, revealing how the mass segregation can affect the nitrogen migration.

\subsubsection{Influence of the number of pulses}

The raw beam laser treatment has been performed in 1 bar $\mathrm{N}_{2}$ at the fluence of $5 \mathrm{~J} / \mathrm{cm}^{2}$, ranging the number of pulses between 2 and 256. The FT of the Si sample irradiated 256 times is reported in Fig. 7.9 with the FT of the virgin $\mathrm{Si}$ and the $\alpha-\mathrm{Si}_{3} \mathrm{~N}_{4}$ standard. The laser-irradiated sample reveals all the peaks belonging to the Si structure, but only the first shell of the Si nitride phase is observable, suggesting that the nitride is highly disordered or amorphous (the poor crystallinity of the nitride phase was confirmed by XRD, where only the peaks of polycrystalline Si have been observed). The BFT procedure was performed in the region $0.86 \AA<R<2.46 \AA$ including the peaks corresponding to 


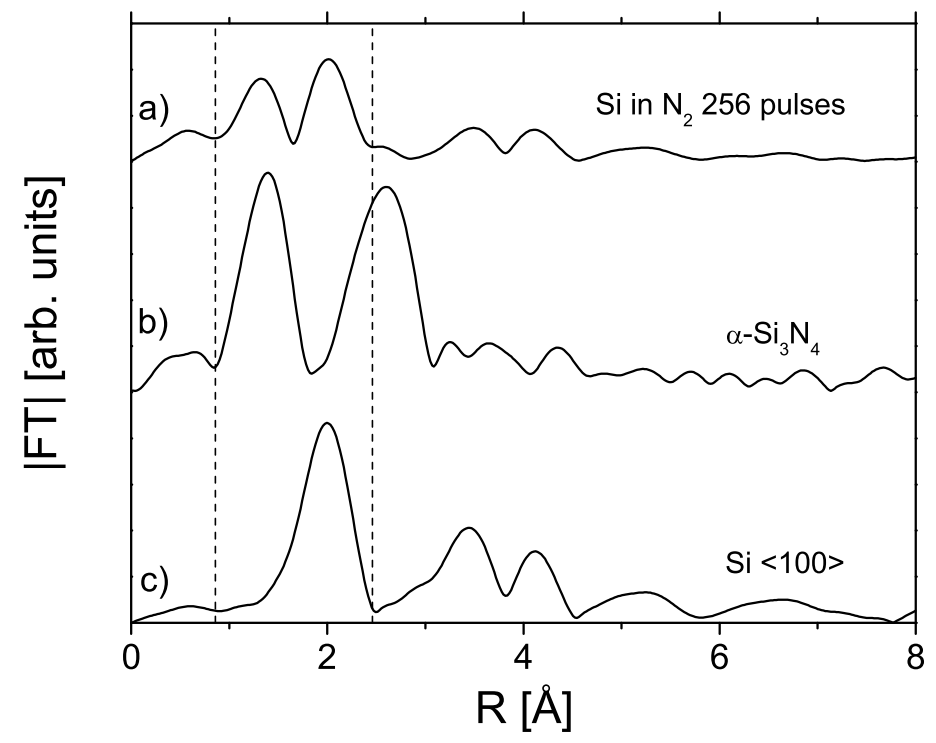

Figure 7.9: Fourier transform moduli of: a) Si sample irradiated in $\mathrm{N}_{2}$ with 256 pulses, b) $\alpha-\mathrm{Si}_{3} \mathrm{~N}_{4}$ standard, c) virgin $\mathrm{Si}<100>$. The dashed vertical lines delimit the BFT region.

the first shell of the $\mathrm{Si}_{3} \mathrm{~N}_{4}$ system (N scatterer, peak at $\sim 1.35 \AA$ ) and the first shell of $\mathrm{Si}$ (peak at $\sim 2.05 \AA$ ). As for the $\mathrm{SiC}$ analysis, the scattering amplitudes and the phases of each shell were obtained from the standard samples (the virgin $\mathrm{Si}$ and the $\alpha-\mathrm{Si}_{3} \mathrm{~N}_{4}$ powder) and loaded in the fits as initial parameters. Since the absorption edges of Si and $\mathrm{Si}_{3} \mathrm{~N}_{4}$ differ by $4 \mathrm{eV}(1839 \mathrm{eV}$ and $1843 \mathrm{eV}$, respectively), this energy correction has been introduced as a fixed parameter in the fitting procedure of the first shell of $\mathrm{Si}_{3} \mathrm{~N}_{4}$. The oscillations extrapolated from the BFT of the silicon sample irradiated with 256 laser shots are shown in Fig. 7.10. The disorder parameter $\Delta \sigma^{2}$ (relative to the standard) of the Si shell was found to be less than $4 \times 10^{-4} \AA^{2}$, but the average value of $2.8 \times 10^{-3} \AA^{2}$ has been obtained for the $\mathrm{Si}_{3} \mathrm{~N}_{4}$ shell, indicating a larger degree of disorder in the laser-synthesized nitride. The number of neighbors were normalized with respect to the ideal values of the pure phases, in order to evaluate the phase ratios. There are four $\mathrm{N}$ neighbors in the first shell of $\mathrm{Si}_{3} \mathrm{~N}_{4}$ in a distorted tetrahedral configuration, at a distance of $1.7 \AA-1.8 \AA$, according to the kind of polymorph [125, 126]. The radius of the first nitride shell obtained from the BFT fitting is consistent with the value of the $\alpha-\mathrm{Si}_{3} \mathrm{~N}_{4}$ phase, and the coordination numbers of both the $\mathrm{Si}$ and the $\mathrm{Si}_{3} \mathrm{~N}_{4}$ shells have been used to evaluate the $\mathrm{Si}_{3} \mathrm{~N}_{4}$ fraction with a procedure analogous to the one used for the $\mathrm{SiC}$ samples. The results of the pulse series are shown in Fig. 7.11 and summarized in Table 7.2. It should be noticed that the relative amount of the nitride phase saturates after few laser shots to values between $40 \%$ and $50 \%$. As it will be shown is the next section, this feature is in qualitative agreement with the RNRA observation, where a sharp nitrogen peak can be observed even after only 4 laser shots (see Fig. 7.13). The nitrogen depth profiling revealed the strong influence of the meandering treatment on the mass transport. Opposite to the case of $\mathrm{SiC}$, as reported in the inset of Fig. 7.12, the nitrogen content on the surface of the sample meandered with $16 \times 16$ spot overlap is almost half 




Figure 7.10: Back Fourier transform oscillations of the Si sample irradiated with 256 laser pulses in 1 bar $\mathrm{N}_{2}$.

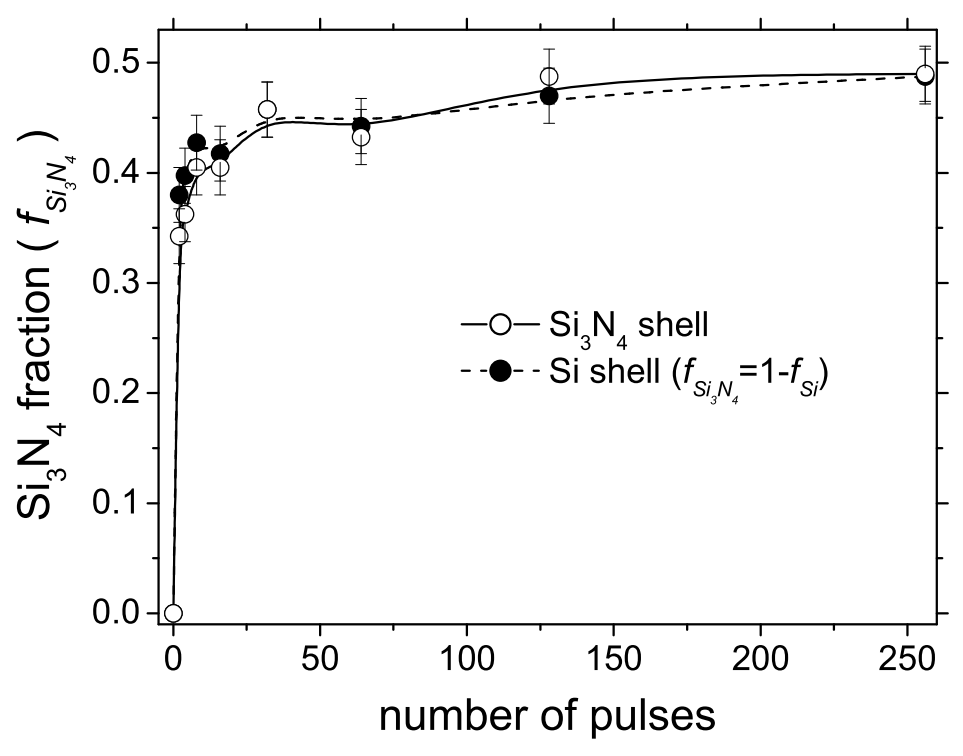

Figure 7.11: $\mathrm{Si}_{3} \mathrm{~N}_{4}$ phase fraction obtained from the EXAFS analysis as function of the number of laser pulses: (o) first shell of $\mathrm{Si}_{3} \mathrm{~N}_{4},(\bullet)$ first shell of Si.

compared to the amount of nitrogen in the sample treated under identical conditions but as a single spot with 256 laser pulses. This is in qualitative agreement with the intensity ratio of the $\mathrm{Si}$ and the $\mathrm{Si}_{3} \mathrm{~N}_{4}$ peaks obtained from the FT of the EXAFS data, as reported in Fig. 7.12. The sharp nitride surface peak ( $\sim 20 \mathrm{~nm}$ thick) obtained with the single spot 
Table 7.2: Two-shells fitting for the Si samples irradiated in $\mathrm{N}_{2}$ : summary of the results.

\begin{tabular}{ccccc}
\hline \hline Laser shots & \multicolumn{2}{c}{ Si-N (I shell) } & \multicolumn{2}{c}{ Si (I shell) } \\
& $\mathrm{N} / 4$ & $\mathrm{R}[\AA]$ & $\mathrm{N} / 4$ & $\mathrm{R}[\AA]$ \\
\hline 2 & 0.343 & 1.73 & 0.620 & 2.35 \\
4 & 0.363 & 1.72 & 0.603 & 2.35 \\
8 & 0.405 & 1.72 & 0.573 & 2.35 \\
16 & 0.405 & 1.73 & 0.583 & 2.36 \\
32 & 0.458 & 1.73 & 0.543 & 2.36 \\
64 & 0.433 & 1.73 & 0.558 & 2.36 \\
128 & 0.488 & 1.73 & 0.530 & 2.36 \\
256 & 0.490 & 1.73 & 0.513 & 2.35 \\
\hline \hline
\end{tabular}



Figure 7.12: Fourier transform moduli of the samples irradiated in $\mathrm{N}_{2}$ : single spot with 256 pulses (solid line) and the $16 \times 16$ scan (dashed line). The inset show the corresponding nitrogen depth profiles measured by RNRA. The vertical dotted line is the average information depth of the EXAFS in TEY mode $(\sim 30 \mathrm{~nm})$.

treatment is attributed to the strong segregation of nitrogen in Si during the cooling of the substrate. The meandering treatment performed under the same experimental conditions enhances the migration of nitrogen to larger depth, nevertheless the EXAFS oscillations of the sample treated as single spot and the meandered one have similar features, revealing only the first shell of the $\mathrm{Si}_{3} \mathrm{~N}_{4}$ and all peaks of $\mathrm{Si}$. This suggests that the different mechanism of nitrogen migration does not lead to a different crystallization of the nitride phase. The complete absence of the second shell of $\mathrm{Si}_{3} \mathrm{~N}_{4}$ in the laser nitrided samples suggests an alternative explanation of the EXAFS results. As reported by various authors [127, 128, 129], nitrogen in silicon is known to exist mostly in molecular form. Theoretical 
calculations confirmed by experimental evidence have shown that the nitrogen-pair defect can be present in the ideal Si structure forming Si-N bonds with length of $1.7 \AA-1.8 \AA$. In such a case, the EXAFS oscillations would have the contributions from the $\mathrm{N}$ scatterers with a shell radius of $1.7 \AA-1.8 \AA$ and the contributions from all the shells of Si. Since the Si structure is not strongly affected by the nitrogen pair [127], the coordination number of the first Si shell should be four-fold (i.e. the ideal value). Nevertheless, the FT of the laser irradiated samples reveals a clear decrease of the number of Si neighbors, suggesting the presence of vacancies $(\mathrm{V})$. The existence of the vacancy-nitrogen complex has been extensively studied [129, 130, 131], demonstrating that the configuration $\mathrm{N}_{2}-\mathrm{V}_{2}$ is more stable than the nitrogen-pair defect with a theoretical Si-N bond of length $\sim 1.8 \AA$.

\subsubsection{The segregation problem}

With the single spot treatment in nitrogen atmosphere, the nitride layer has a thickness of only a few tens of nanometers. As already mentioned in the previous section, the fact that the nitrogen does not diffuse deeper into the silicon matrix is attributed to segregation. This phenomenon occurs when the impurities (in this case N) are rejected by the advancing solid/liquid interface in the phase characterized by the higher solubility, i.e. the liquid, and then accumulate at the sample surface [132, 133]. A clear indication of the segregation phenomenon is reported in Fig. 7.13. The nitrogen depth profiles measured by RNRA for various number of laser shots show the same characteristic surface peak and very little nitrogen remains inside the sample. Even if during the laser melting some nitrogen diffuses in the liquid silicon, as the solid/liquid interface recedes toward the surface, the nitrogen "jumps" from the solidified portion into the liquid layer, eventually accumulating on the target surface. The analytical description of the segregation process assumes that the redistribution of impurities is governed by the diffusion equation [133]:

$$
\frac{\partial C}{\partial t}=\frac{\partial}{\partial z}\left(D \frac{\partial C}{\partial z}\right)
$$

where $D$ is the diffusion constant and $C$ is the impurity concentration. The boundary conditions are a reflecting surface:

$$
\left.\frac{\partial C}{\partial z}\right|_{z=0}=0
$$

and a moving solid/liquid interface that rejects the impurities in the liquid:

$$
D \frac{\partial C}{\partial z}+\left.(1-K) v_{s l} C\right|_{z_{i}}=0
$$

with $v_{s l}$ being the solid/liquid interface velocity and $z_{i}$ its instantaneous position. The segregation coefficient:

$$
K=\left.\frac{C_{s}}{C_{l}}\right|_{z_{i}}<1
$$

is the solubility ratio at the interface $\left(C_{s}\right.$ and $C_{l}$ are the impurities concentrations in the solid and the liquid, respectively). Eqs. (7.1) to (7.4) can be solved analytically [134, 


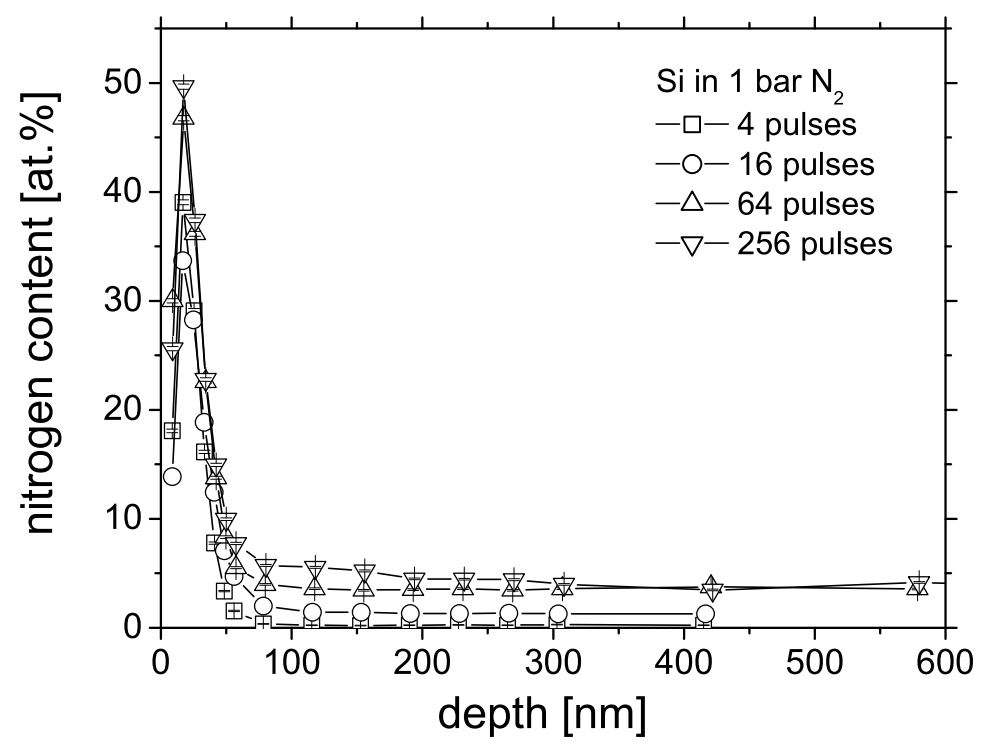

Figure 7.13: RNRA nitrogen depth profiles of Si treated as single spot with laser fluence of 5 $\mathrm{J} / \mathrm{cm}^{2}$ in 1 bar $\mathrm{N}_{2}$ and various number of pulses.

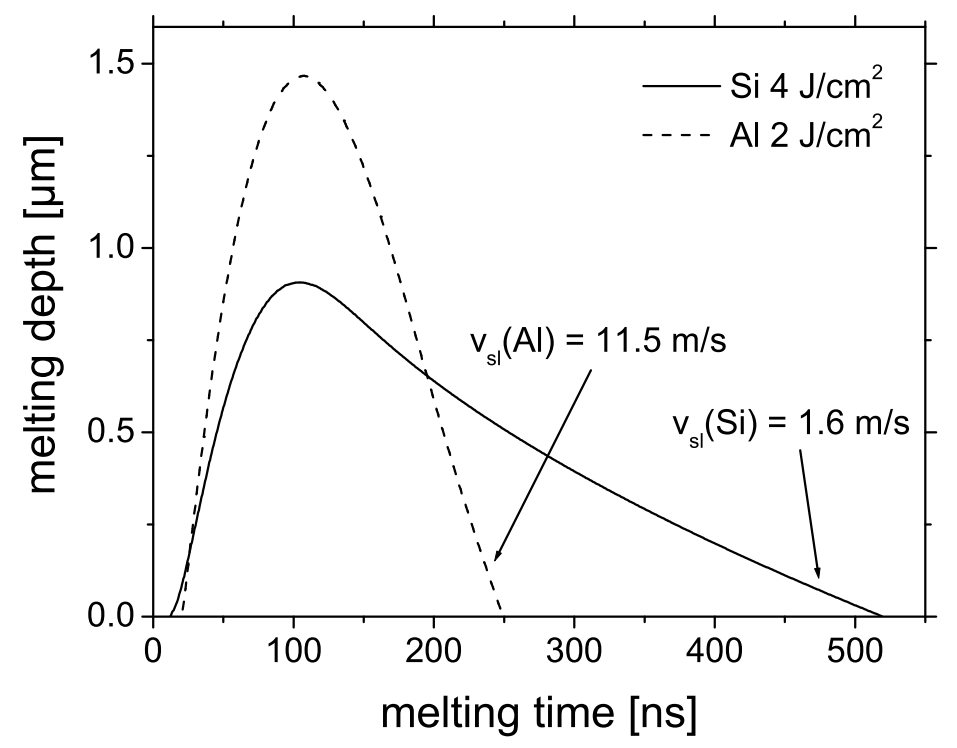

Figure 7.14: The melting depths vs. melting times of a Si substrate irradiated at $4 \mathrm{~J} / \mathrm{cm}^{2}$ and an $\mathrm{Al}$ substrate irradiated at $2 \mathrm{~J} / \mathrm{cm}^{2}$ : the interface velocity $v_{s l}$ is the slope of the curve calculated where indicated by the arrows.

revealing the strong influence of the interface velocity on the segregation effect. In particular, small values of $v_{s l}$ enhance the surface accumulation of the impurities, provided that the segregation coefficient is small enough $(K<0.1)$. The segregation of several 
dopants in silicon after pulsed laser irradiation has been extensively studied (see [132] and references therein), but unfortunately no data are available on the segregation of $\mathrm{N}$ in Si. As a further proof, Fig. 7.14 reports the comparison between the solid/liquid interface velocities of the $\mathrm{Al}$ substrate irradiated at $2 \mathrm{~J} / \mathrm{cm}^{2}$ and the Si substrate irradiated at $4 \mathrm{~J} / \mathrm{cm}^{2}$. The difference is almost one order of magnitude $\left(v_{s l}(\mathrm{Al})=11.5 \mathrm{~m} / \mathrm{s}\right.$, $\left.v_{s l}(\mathrm{Si})=1.6 \mathrm{~m} / \mathrm{s}\right)$, indicating that in the semiconductor the segregation is more effective. A possible way to avoid the accumulation of $\mathrm{N}$ on the target surface is the meander scan. As already pointed out in Fig. 7.12, opposite to the single spot treatment, the meandering scan allows the migration of $\mathrm{N}$ (or $\mathrm{Si}_{3} \mathrm{~N}_{4}$ ) to larger depth. In this case the origin of the nitrogen depth profiles is correlated to the strong convection induced by the piston effect [3, 28] at the edge of the laser spot: as schematically illustrated in Fig. 7.15, even if the

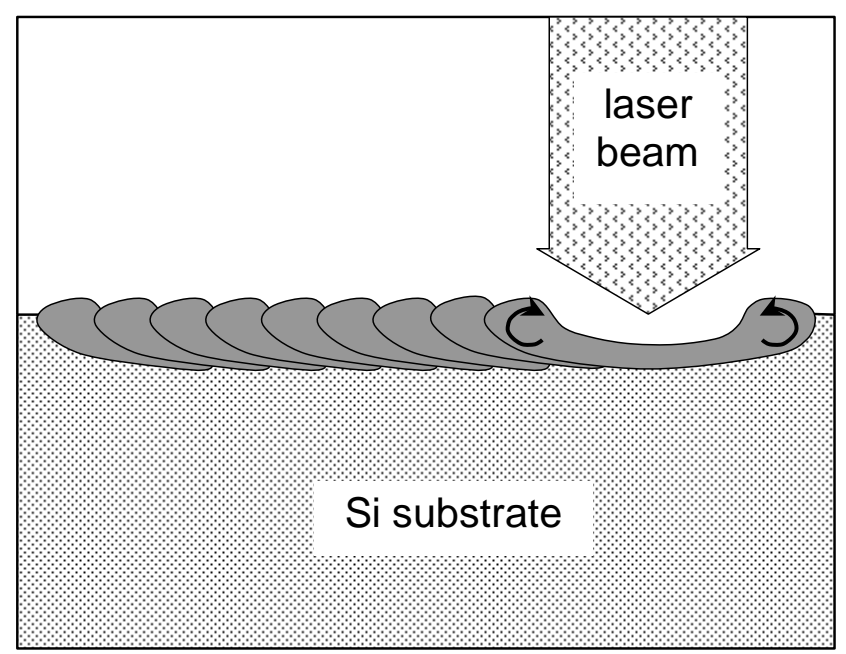

Figure 7.15: Sketch of the mass transport mechanism during the meander treatment with the raw laser beam: the convection due to the piston effect is indicated by the arrows.

segregation is present, it should interest mainly the center of the laser spot, while at the borders of the irradiated area, the mass transport should be dominated by convection. The meandering scan leads to an inevitable overlap of the laser spot borders, and the segregation effect is "masked" by the mass convection. The RNRA nitrogen profiles of the samples irradiated with the $16 \times 16$ scan in 1 bar $\mathrm{N}_{2}$ at different laser fluences are reported in Fig. 7.16. Apart the very surface (within $50 \mathrm{~nm}$ ), these depth profiles resemble the carbon profiles obtained under similar experimental conditions (meander scans with analogous laser fluence and gas pressures, see insets in Figs. 7.6 and 7.8). For a laser fluence below $3 \mathrm{~J} / \mathrm{cm}^{2}$ the nitriding efficiency is quite poor, but the threshold rises to $4 \mathrm{~J} / \mathrm{cm}^{2}$ if the homogenized beam is used. This difference is caused by the inhomogeneity of the raw beam intensity distribution: the average fluence is $3 \mathrm{~J} / \mathrm{cm}^{2}$, but the fluctuations can be as large as $\pm 1 \mathrm{~J} / \mathrm{cm}^{2}$ over the laser spot. A concluding remark concerns the carbon incorporation into silicon. According to the EXAFS and the RBS analyses, the carbon does not form any sharp surface peak on the silicon surface and migrates to large depth during the single spot treatment, suggesting that the segregation is absent. As pointed out in section 2.3 the carbon atoms dissolve substitutionally in Si forming $\mathrm{SiC}$, and not interstitially like nitrogen. Therefore, the impurities ( $\mathrm{C}$ atoms) in such a case form a stable phase and they do not jump from the solid to the liquid as the interface 




Figure 7.16: RNRA nitrogen depth profiles of the Si samples meandered with the $16 \times 16$ scan at various laser fluences (from $2 \mathrm{~J} / \mathrm{cm}^{2}$ to $5 \mathrm{~J} / \mathrm{cm}^{5}$ ).

moves toward the target surface. 


\section{Chapter 8}

\section{Conclusions and outlooks}

The laser processing of iron, aluminum and silicon substrates in nitrogen and methane atmospheres has been the subject of this work. The mass transport mechanisms during the laser irradiation in nitrogen gas have been studied in detailed, revealing the following facts:

(i) the mobility of nitrogen inside the iron matrix can be attributed to diffusion in the liquid metal: thanks to the capability of the RNRA, the contribution of each laser pulse to the nitrogen incorporation and to the subsequent migration could be quantified, shedding light on the nitrogen incorporation mechanism and its relation with the phenomena occurring inside the target (temperature, melting time, melting depth) and on the target surface (plasma formation). Besides, the nitrogen losses due to the degassing and to the ablation effects could be revealed;

(ii) nitrogen can be efficiently incorporated in the aluminum matrix, if the excimer laser fluence exceeds $2 \mathrm{~J} / \mathrm{cm}^{2}$; the nitrogen transport mechanism is strongly related to the local temperature induced by the absorbed laser energy: when the irradiated sample is heated above the melting/dissociation point of AlN $(\sim 3000 \mathrm{~K})$, atomic nitrogen can rapidly migrate in the liquid metal, forming homogeneous depth concentrations that are a direct consequence of two competing contributions: an inward flux due to the nitrogen incorporation and an outward flux due to the nitrogen outgassing; the quantification of the nitrogen content as a function of the number of laser shots and the nitrogen gas pressure has been successfully obtained. It should be mentioned that after 256 pulses in 6 bar nitrogen gas, a homogeneous AlN layer is formed. When the temperature of the irradiated sample is above the melting point of $\mathrm{Al}$, but not high enough to dissociate the AlN $(\sim 2000 \mathrm{~K})$, the latter (and not the atomic nitrogen) drifts in the molten aluminum layer due to the driving forces induced by the Brownian motion and the thermophoresis, which in turn are related to the chemical and the thermal gradients, respectively. The mechanical properties of the nitrided samples revealed a considerable increase of the surface hardness, closely correlated to the nitrogen content;

(iii) the nitrogen incorporation in the silicon substrates is strongly affected by the chemical and physical properties of the elements involved in the process. During the single spot irradiation, the segregation effect hinders the migration of nitrogen which instead accumulates on the surface of the target. The laser nitriding process becomes more efficient if 
the meandering scan is employed. In this case the nitrogen/nitride transport mechanism is attributed to the mass convection induced by the piston effect. The poor crystallinity of the laser-synthesized silicon nitride and the results of the EXAFS analysis suggest the formation of the nitrogen-vacancy complex $\mathrm{N}_{2}-\mathrm{V}_{2}$.

The laser treatment in methane atmosphere has been investigated here for the first time. The attention has been focused on the structural modifications induced by the laser irradiation, and the results can be summarized as follows:

(i) the carbon incorporation in iron is effective: the phase evolution as a function of the methane pressure and the laser scan has been investigated in detail with Mössbauer spectroscopy and X-ray diffraction. At a laser fluence of $4 \mathrm{~J} / \mathrm{cm}^{2}$, with intermediate methane pressures (few bar) and moderate spot overlap $(\leq 11 \times 12)$ a mixture of $\gamma$-Fe $(\mathrm{C}), \epsilon$ phase and cementite $\left(\theta-\mathrm{Fe}_{3} \mathrm{C}\right)$ is obtained. Increasing the gas pressure or the meander overlap produces equivalent effects, leading to the formation of a homogeneous cementite layer, as revealed by all analytical techniques. The synthesis of $\theta-\mathrm{Fe}_{3} \mathrm{C}$ layers is technologically a rather important result: this phase is typically embedded in the metal matrix and it is hard to obtain as a single phase. Nanoindentation measurements revealed that the mechanical properties of the carburized layer are largely enhanced by the laser process;

(ii) carbon can be incorporated also in the aluminum matrix, forming amorphous aluminum carbide $\left(\mathrm{a}-\mathrm{Al}_{4} \mathrm{C}_{3}\right)$; the crystallization of the carbide phase is most probably hindered by the strong thermal gradients produced during the laser treatment. Nevertheless a considerable amount of carbon $(\sim 20$ at.\%) and a good increase of the surface hardness have been observed;

(iii) by irradiating silicon substrates in methane atmosphere, the formation of polycrystalline, cubic silicon carbide $(\beta$-SiC) with good degree of crystallinity has been successfully achieved, as indicated by the EXAFS results. The strong influence of the laser scan (single spot or meandering) on the carbon migration has been revealed. The surface carbon concentration is higher, if the meandering treatment is employed, but the carbon profiles are more homogeneous after single spot irradiations. Opposite to the treatment in nitrogen gas, no mass segregation has been observed, and the intriguing fact that the carbon incorporation is more effective at lower methane pressure indicates the important role of the gas pressure on the carburizing efficiency. It should be mentioned that the carbide phase is mixed with the non-reacted silicon matrix, and the optimization of the laser process aims to the maximization of the $\mathrm{SiC}$ relative fraction.

The detailed investigation of the carbon transport mechanism in the irradiated targets requires the use of RNRA with the ${ }^{13} \mathrm{C}(\mathrm{p}, \gamma){ }^{14} \mathrm{~N}$ nuclear reaction at resonance energy $E_{r}=$ $1748 \mathrm{keV}$. For this purpose, the capability of the $3 \mathrm{MeV}$ Pelletron accelerator facility at the Zweites Physikalisches Institut in Göttingen should considerably increase the investigative power. This is considered one of the primary tasks for the future investigation of the laser carburazing process. Understanding the mass transport during the laser irradiation, should give important hints on the the carbon incorporation mechanism. The results of the present work also reveal the fundamental role of the phenomena occurring on the surface of the irradiated target. The vaporization of the substrate, the ionization of the ambient gas and the formation of a plasma have been predicted theoretically, but their direct observation has not been carried out yet. The investigation and characterization of these phenomena is not straightforward and requires complex experiments. For this reason, in 
situ, time-resolved analyses by means of optical, UV and X-ray spectroscopy have been planned, in order to shed light on the plasma-vapor interaction with the substrate. On the other hand, the influence of other experimental parameters such as the duration and wavelength of the laser pulse and the use of alternative gaseous atmospheres $\left(\mathrm{NH}_{3}\right.$ instead of $\mathrm{N}_{2}, \mathrm{C}_{2} \mathrm{H}_{2}$ instead of $\mathrm{CH}_{4}$ ) are currently under investigation. 


\section{Acknowledgements}

I'm deeply grateful to PD Dr. Peter Schaaf for his constant and unexhaustable help, and I truly thank him for the big scientific freedom he always provided, without which most of this work would not exist.

I'm also in debt to Prof. Dr. Klaus-Peter Lieb for his continuous interest and support to this topic.

I address my thanks to the former members of the group, Felix Landry, Meng Han and Steffen Wagner for their valuable help in the experimental work and for the nice atmosphere they created inside the group.

Marcus Schwickert and Felix Harbsmeier are gratefully acknowledged for being excellent officemates and good beer-drinkers. I'm in dept to Felix for introducing me to the IONAS setup and to Marcus for the numerous evening we spent in the office, working, simulating, analyzing and, lately, writing.

Many thanks to Alexander Müller and Sankar Dhar for their help and for the fruitful discussions: Sankar and his wife are gratefully acknowledged for the excellent "spicy dinner" they are capable of.

I'm grateful to the Herwig Schultz, Klaus Arndt, head of the fine mechanics workshop, Kurt Schemmerling, head of the electronic workshop, and all their coworkers for the efficient, competent and prompt job. Many thanks to Detlef Purschke for operating the IONAS facility and to Dr. Lüder Ziegler for the handling of the Mössbauer radioactive sources.

I'm in debt to Dr. Guido Schmitz from the Institut für Materialphysik in Göttingen for the preparation and the Transmission Electron Microscopy observation of the AlN samples. Dieter Plischke is gratefully acknowledged for operating the Scanning Electron Microscopy and for his patience during the experimental observations.

I'm obliged to Dr. Nikita Arnold from Linz University for the interesting and fruitful discussion concerning the laser interaction with matter and the diffusion problem. Many thanks also to Prof. Dr. Andreas Emmel from the Fachhochschule Amberg-Weiden for the nice suggestions about the laser nitriding of aluminum.

Frau Christa Wohlfahrt and Lucie Hamdi are kindly acknowledged for their continuous help with all the small and big administrative problems that a foreigner always has in a foreign country. 
Dr. Anne-Marie Flank, Dr. Pierre Lagarde and Dr. Agnes Traverse are acknowledged for their assistance at the L.U.R.E. (Orsay) during our XAFS beamtime and for the illuminating advice concerning the data analysis.

My sincere thanks to all the members of the Zweites Physikalisches Institut in Göttingen, who always make the working atmosphere enjoyable and relaxed with extra-time activities (barbecues, kicker-table competitions, outdoor trips).

Finally, my thoughts inevitably go to my parents, my family and to all those persons who always, critically, constructively supported my choices and my work. To them I address my deepest gratitude and the best wishes for a brilliant continuation.

Göttingen, september 2002 


\section{Bibliography}

[1] A. Sona, in Applied Laser Tooling, edited by O. D. D. Soares and M. Perez-Amor (M. Nijhoff, Dordrecht, 1987).

[2] E. V. Locke and R. A. Hella, IEEE J. 10, 179 (1974).

[3] M. von Allmen and A. Blatter, Laser-Beam Interactions with Materials (Springer-Verlag, Berlin, 1998).

[4] W. M. Steen, Laser Material Processing (Springer, Berlin, 1998).

[5] D. Bäuerle, Laser Processing and Chemistry (Springer-Verlag, Heidelberg, 2000).

[6] H. K. Tönshoff et al., Int. J. Elect. Machining 4, 1 (1999).

[7] S. Küper and M. Stuke, Microelect. Eng. 9, 475 (1989).

[8] M. Hegelich et al., Phys. Rev. Lett. 89, 0850021 (2002).

[9] Physical Metallurgy, edited by R. W. Cahn and P. Haasen (North-Holland, Amsterdam, 1983).

[10] E. Lehrer, Z. Electrochem. 36, 383 (1930).

[11] T. Fujii, K. Yoshida, K. Suzuki, and S. Ito, Solid State Ionics 141-142, 593 (2001).

[12] K. Tanihata and Y. Miyamoto, Int. J. Self Propagating Heat Temperature Synthesis 7, 209 (1998).

[13] H. J. Kim and Y. G. Kweon, Wear 174, 201 (1994).

[14] M. H. Jacobs and Y. G. Kweon, Materials and Design 14, 33 (1993).

[15] A. L. Thomann et al., J. Appl. Phys. 80, 4673 (1996).

[16] S.-M. Oh and D.-W. Park, Thin Solid Films 319, 189 (1998).

[17] M. Yan, J. Yan, and T. Bell, Modelling Simul. Mat. Sci. Eng. 8, 491 (2000).

[18] V. I. Dimitrov et al., Comput. Mat. Sci. 15, 22 (1999).

[19] Y. Sun and T. Bell, Mat. Sci. Eng. A224, 33 (1997).

[20] E. D. Cabanillas, J. Desimoni, G. Punte, and R. C. Mercader, Mat. Sci. Eng. A279, 133 (2000).

[21] K. C. Walters, J. Vac. Sci. Technol. B12, 945 (1994).

[22] T. Reier, J. W. Schultze, W. Österle, and C. Buchal, Thin Solid Films 385, 29 (2001).

[23] T. Telbizova et al., Appl. Phys. Lett. 76, 1404 (2000).

[24] E. C. Moreira, M. Behar, and L. Amaral, Nucl. Instr. and Methods B148, 836 (1999).

[25] J. Jagielski and M. Kopcewicz, J. Appl. Phys. 88, 673 (2000).

[26] A. Königer, C. Hammerl, M. Zeitler, and B. Rauschenbach, Phys. Rev. B55, 8143 (1997).

[27] E. C. Paloura, C. Lioutas, and A. Markwitz, J. Appl. Phys. 80, 2720 (1996).

[28] C. Illgner, Untersuchungen zum Lasernitrieren von Eisen (Cuvillier Verlag, Göttingen, 1996), doctoral thesis. 
[29] F. Landry, Lasernitrieren von Armco-Eisen und Eisenwerkstoffen (Cuvillier Verlag, Göttingen, 1999), doctoral thesis.

[30] M. Han, Laser nitriding of metals (http://webdoc.sub.gwdg.de/diss/2002/han/han.pdf, Göttingen, 2001), doctoral thesis.

[31] P. Schaaf, Prog. Mat. Sci. 47, 1 (2002).

[32] Thin Solid Films, edited by J. Kossut (Elsevier Science, The Netherlands, 2002), Vol. 412.

[33] Surface and Coatings Technology, edited by H. Bangert et al. (Elsevier Science, The Netherlands, 2002), Vol. 151-152.

[34] Surface and Coatings Technology, edited by B. Sartwell (Elsevier Science, The Netherlands, 2001), Vol. 140.

[35] Surface and Coatings Technology, edited by C. Mitterer et al. (Elsevier Science, The Netherlands, 2001), Vol. 146-147.

[36] Thin Solid Films, edited by H. Hosono, N. Ichinose, and Y. Shigesato (Elsevier Science, The Netherlands, 2002), Vol. 411.

[37] Thin Solid Films, edited by A. Slauoi, J. Poortmans, A. Jager-Waldau, and C. Brabec (Elsevier Science, The Netherlands, 2002), Vol. 403-404.

[38] Binary Alloy Phase Diagrams, edited by H. O. T .B. Massalski, P. R. Subramanian, and L. Kacprzak (ASM International, Ohio, 1996).

[39] M. Ron, in Applications of Mössbauer Spectroscopy II, edited by R. L. Cohen (Academic Press, New York, 1980).

[40] J. Häglung, G. Grimvall, and T. Jarlborg, Phys. Rev. B44, 2914 (1991).

[41] S. J. Li et al., Thin Solid Films 316, 100 (1998).

[42] J. Kunze, Nitrogen and Carbon in Iron and Steel, Physical Research (Akademie-Verlag, Berlin, 1990), Vol. 16.

[43] Handbook of Chemistry and Physics, edited by R. C. Weast and M. J. Astle (CRC Press, Boca Raton, 1983).

[44] Powder Diffraction File database PDF-2 (International Centre for Diffraction Data, Newton Square, 1997).

[45] H. A. Wriedt, N. A. Gokcen, and R. H. Nafziger, in Binary Alloy Phase Diagrams, edited by T. B. Massalski, H. Okamoto, P. R. Subramanian, and L. Kacprzak (ASM International, Ohio, 1996).

[46] H. Okamoto, J. Phase Eq. 13, 2 (1992).

[47] L. F. Mondolfo, Aluminum Alloys: Structure and Properties (Butterworth, London, 1976).

[48] L. G. D'yachkov, L. A. Zhilyakov, and A. V. Kostanovskii, Thecnical Physics 45, 115 (2000).

[49] D. P. Dandekar, A. Abbate, and J. Frankel, J. Appl. Phys. 76, 4077 (1994).

[50] Goodfellow Metal Ltd., www.goodfellow.com.

[51] S. Strite and H. Morkoç, J. Vac. Sci. Technol. B10, 1237 (1992).

[52] H. Morkoç et al., J. Appl. Phys. 76, 1363 (1994).

[53] N. W. Ashcroft and N. D. Mermin, Solid State Physics (Saunders Company, Philadelphia, 1976).

[54] A. Zerr et al., Nature 400, 340 (1999).

[55] S. Fähler and H. U. Krebs, Appl. Surf. Sci. 96-98, 61 (1996).

[56] J. Crank, The Mathematics of Diffusion (Clarendon Press, Oxford, 1975).

[57] C. A. J. Fletcher, Computational Techniques for Fluid Dynamic 1 (Springer, Berlin, 1988). 
[58] Thermophysical Properties of Matter, edited by Y. S. Touloukian and C. Y. Ho (Plenum, New York, 1970), Vol. 1 and 4.

[59] S. Higashi and T. Sameshima, Jpn. J. Appl. Phys. 40, 480 (2001).

[60] M. Hatano et al., J. Appl. Phys. 87, 36 (2001).

[61] S. I. Anisimov and V. A. Khokhlov, Instabilities in Laser-Matter Interactions (CRC Press, Boca Raton, 1995).

[62] S. I. Anisimov, Sov. Phys. JETP 27, 182 (1968).

[63] Y. P. Raizer, Sov. Phys. JEPT 21, 1009 (1965).

[64] Y. P. Raizer, Sov. Phys. JEPT 31, 1148 (1970).

[65] A. Pirri, Phys. Fluids 16, 1435 (1973).

[66] A. A. Boni and F. Y. Su, Phys. Fluids 17, 340 (1974).

[67] Y. P. Raizer, Sov. Phys. Usp. 23, 789 (1980).

[68] Y. P. Raizer, Sov. Phys. Quantum Electron. 14, 40 (1984).

[69] M. Uhrmacher et al., Nucl. Instr. and Methods B9, 234 (1985).

[70] J. A. Leavitt and J. L. C. McIntyre, in Handbook of Modern Ion Beam Materials Analysis, edited by J. R. Tesmer and M. Nastasi (Material Research Society, Pittsburgh, 1995).

[71] W. H. Bragg and R. Kleeman, Philos. Mag. 10, 318 (1905).

[72] W.-K. Chu, J. W. Mayer, and M.-A. Nicolet, Backscattering Spectrometry (Academic Press, Orlando, 1978).

[73] N. Bohr, Mat. Fys. Medd. Dan. Vid. Selsk. 18, 8 (1948).

[74] J. Lindhard and M. Scharff, Mat. Fys. Medd. Dan. Vid. Selsk. 27, 15 (1953).

[75] L. R. Doolittle, Nucl. Instr. and Methods B9, 344 (1985).

[76] T. Osipowicz, K. Lieb, and S. Brüssermann, Nucl. Instr. and Methods B18, 232 (1987).

[77] G. Schatz and A. Weidinger, Nucleare Festkörperphysik (Teubner, Stuttgart, 1992).

[78] Handbook of Modern Ion Beam Materials Analysis, edited by J. R. Tesmer and M. Nastasi (Material Research Society, Pittsburgh, 1995).

[79] N. N. Greenwood and T. C. Gibb, Mössbauer Spectroscopy (Chapman and Hall, London, 1971).

[80] R. L. Mössbauer, Z. Physik 151, 124 (1958).

[81] R. L. Mössbauer, Naturwisseschaften 45, 538 (1958).

[82] R. L. Mössbauer, Science 137, 731 (1962).

[83] A. Abragam, Principles of Nuclear Magnetism (Clarendon Press, London, 1961).

[84] J. J. Spijkerman, in The Mössbauer Effect Methodology, edited by I. J. Gruverman (Plenum Press, New York, 1970), Vol. 7.

[85] F. E. Wagner, J. de Physique 37, 673 (1976).

[86] J. J. Rehr and R. C. Albers, Rev. Mod. Phys. 72, 621 (2000).

[87] D. E. Sayers and B. A. Bunker, in X-Ray Absorption, edited by D. C. Koningsberger and R. Prins (John Wiley and Sons, New York, 1988), Chap. 6.

[88] D. E. Sayers, E. A. Stern, and F. W. Lytle, Phys. Rev. Lett. 27, 1204 (1971).

[89] A. L. Ankudinov, B. Ravel, J. J. Rehr, and S. D. Conradson, Phys. Rev. B58, 7565 (1998).

[90] W. T. Elam, J. P. Kirkland, R. A. Neiser, and P. D. Wolf, Phys. Rev. B38, 26 (1988).

[91] K. V. Klementev, VIPER for Windows, www.desy.de/ klmn/viper.html. 
[92] K. V. Klementev, J. Phys. D: Appl. Phys. 34, 209 (2001).

[93] B. D. Cullity, Elements of X-Ray Diffraction (Addison-Wesley, Reading, 1978).

[94] H. M. Rietveld, Acta Cryst. 22, 151 (1967).

[95] H. M. Rietveld, J. Appl. Cry. 2, 65 (1969).

[96] J. R. Caravajal, FULLPROF, bali.saclay.cea.fr/pub/divers/fullp.

[97] R. A. Young, The Rietveld Method (Oxford University Press, Oxford, 1995).

[98] E. Carpene and P. Schaaf, Appl. Surf. Sci. 186, 100 (2002).

[99] E. Carpene et al., Appl. Surf. Sci 186, 195 (2002).

[100] P. Schaaf, M. Han, K.-P. Lieb, and E. Carpene, Appl. Phys. Lett. 80, 1091 (2002).

[101] E. Carpene, F. Landry, and P. Schaaf, Appl. Phys. Lett. 77, 2412 (2000).

[102] E. Carpene and P. Schaaf, Appl. Phys. Lett. 80, 891 (2002).

[103] E. Carpene, M. Kahle, M. Han, and P. Schaaf, in Mössbauer Spectroscopy in Materials Science, edited by M. Miglierini, M. Mashlan, and P. Schaaf (Kluwer Academic Publishers, Dordrecht, 2002), NATO Science Series "High Technologies", in print.

[104] P. Schaaf, F. Landry, and K. Lieb, Appl. Phys. Lett. 74, 153 (1999).

[105] E. Fromm et al., Gases and Carbon in Metals (Physik Daten FIZ Energie, Physik, Mathematik, Karlsruhe, 1982), Vol. XV.

[106] J. P. Reilly, A. Ballantyne, and J. A. Woodroffe, AIAA Journal 17, 1098 (1979).

[107] C. Illgner et al., J. Appl. Phys. 83, 2907 (1998).

[108] G. J. Willems and H. E. Maes, J. Appl. Phys. 73, 3256 (1993).

[109] Z. Mathalone, M. Ron, and H. Shechter, Appl. Phys. Lett. 17, 32 (1970).

[110] B. Jönsson and S. Hogmark, Thin Solid Films 114, 257 (1984).

[111] M. Han et al., J. Appl. Phys. 89, 4619 (2001).

[112] M. Ron and Z. Mathalone, Phys. Rev. B4, 774 (1971).

[113] J. Barnikel, Nitrieren von Aluminiumwerkstoffen mit UV-Laserstrahlung (Universität ErlangenNürnberg, Erlangen, 1998), doctoral thesis.

[114] E. Sicard, C. Boulmer-Leborgne, C. Andreazza-Vignolle, and M. Frainais, Appl. Phys. A73, 55 (2001).

[115] C. Boulmer-Leborgne et al., Appl. Surf. Sci. 125, 137 (1998).

[116] E. Carpene and P. Schaaf, Phys. Rev. B65, 224111 (2002).

[117] S. T. Picraux and D. M. Follstaedt, in Surface Modifications and Alloying: Aluminium, edited by J. M. Poate, G. Foti, and D. C. Jacobson (Plenum Press, New York, 1983).

[118] K. Huang, Statistical Mechanics (Wiley and Son, New York, 1987).

[119] A. I. Burshtein, Introduction to Thermodynamics and Kinetic Theory of Matter (Wiley and Son, New York, 1996).

[120] M. Sun, H. Geng, X. Bian, and Y. Liu, Mat. Sci. Forum 20, 337 (2000).

[121] Y. I. Yalamov and A. S. Sanasaryan, Sov. Phys. Tech. Phys. 20, 1351 (1976).

[122] E. R. Shchukin, Y. I. Yalamov, and O. A. Popov, Sov. Phys. Dokl. 32, 898 (1987).

[123] W. R. L. Lambrecht and B. Segall, Phys. Rev. B43, 7070 (1991).

[124] E. Carpene, A. M. Flank, A. Traverse, and P. Schaaf, J. Phys. D: Appl. Phys. 35, 1428 (2002).

[125] P. Yang, H.-K. Fun, I. Rahman, and M. I. Saleh, Ceram. Int. 21, 137 (1995). 
[126] H. Toraya, J. Appl. Cryst. 33, 95 (2000).

[127] R. Jones, S. Öberg, F. B. Rasmussen, and B. B. Nielsen, Phys. Rev. Lett. 72, 1882 (1994).

[128] V. V. Voronkov et al., J. Appl. Phys. 89, 4289 (2001).

[129] H. Sawada and K. Kawakami, Phys. Rev. B62, 1851 (2000).

[130] H. Kageshima, A. Taguchi, and K. Wada, J. Appl. Phys. 76, 3718 (2000).

[131] K. Tanahashi, H. Harada, A. Koukitsu, and N. Inoue, J. Cryst. Growth 225, 294 (2001).

[132] S. U. Campisano, Appl. Phys. A30, 195 (1983).

[133] P. Baeri and S. U. Campisano, in Laser Annealing of Semiconductors, edited by J. M. Poate and J. W. Mayer (Academic Press, New York, 1982).

[134] E. J. Yoffa, in Laser and Electron Beam Processing of Materials, edited by C. W. White and P. S. Peercy (Academic Press, New York, 1980). 


\section{Curriculum studiorum}

\section{Personal Data}

- name: Ettore Carpene

- birthplace: Feltre (BL) - Italy

- $\quad$ birthdate: 22.01 .1971

- citizenship: Italian

\section{Education}

- 1977-1985: Elementary and Middle Schools (Italy)

- 1985-1990: Scientific High School (Italy)

- 1990: High School Diploma "Maturitá Scientifica"

- 1990-1996: Physics studies at the University of Padova (Italy)

- 1996: "Laurea" Degree in Physics

- 1997-1998: Military Service

- 1998-1999: Physics studies at the Old Dominion University in Norfolk, Virginia (USA)

- 1999: Master's Degree of Science

- 1999-2002: Physics PhD student at the II. Physikalisches Institut, University of Göttingen (Germany)

\section{Teaching activities}

- 1998-1999: Teaching assistant at the Old Dominion University in Norfolk, Virginia (USA)

- 2002: Fortgeschrittenenpraktikum at the II. Physikalisches Institut, University of Göttingen (Germany) 